\title{
The following abstracts were presented as posters at the 2016 NEI Psychopharmacology Congress
}

Congratulations to the scientific poster winners of the 2016 NEI Psychopharmacology Congress!

$\mathbf{1}^{\text {st }}$ Place: A Case Report and Literature Review of Clozapine Re-challenge Titration Schedules (page 26)

$2^{\text {nd }}$ Place: Mental Health Statistics and Suicide Prevention at St. Tammany Parish Jail-2015 (page 41)

$3^{\text {rd }}$ Place: Lithium-Induced Neurotoxicity at Therapeutic Range: Illustrative Cases and Literature Review (page 43)

101

\section{Sertraline-induced chemosensory dysfunction}

Ahmed Elazzab'; Alan R. Hirsch, $M D^{2}$; and Wannapak Richter, $\mathrm{MD}^{3}$

${ }^{1}$ Mansoura-Manchester Medical Programme, Mansoura University, Mansoura, Egypt

${ }^{2}$ Smell and Taste Treatment and Research Foundation

${ }^{3}$ Ramathibodi Hospital, Thailand

ABSTRACT: Introduction: Myriad medications can cause chemosensory dysfunction including the antidepressant, citalopram (Okeke et al 2016). Sertraline has not heretofore been reported to cause smell and taste loss.

METHODS: Case study: A 52 year old right handed female was nasute until five years prior to presentation, when she began on citalopram for treatment of depression. Shortly thereafter, she lost her smell and taste, which recovered after discontinuing this antidepressant. A similar situation occurred with sertraline treatment, causing her smell and taste to diminish. On discontinuing sertraline, her smell and taste partially returned which again attenuated after sertraline rechallenge. Specifically before sertraline taste was $80 \%$ of normal, but 2 weeks after $50 \mathrm{mg}$ /day sertraline, taste dropped to $20 \%$. Before sertraline, chocolate taste was $60 \%$ of normal, and then dropped to $10 \%$ of normal on sertraline. Eggroll and fried rice possessed $100 \%$ of normal taste when off sertraline then dropped to 50 and $0 \%$ respectively on sertraline. Coffee was at $75 \%$ taste when off sertraline and $50 \%$ on sertraline, whereas, blue raspberry sucker was $150 \%$ off and $75 \%$ on sertraline. Before sertraline she would experience 5 olfactory windows a day, whereas, on sertraline, she had none.

RESULTS: Name of the test Off sertraline On sertraline Quick Smell Identification test 3 (normal) 1 (anosmia) Alcohol Sniff Test 3 (anosmia) 0 (anosmia) Retronasal Smell Index 2 (abnormal) 1 (abnormal)
DISCUSSION: In this patient impairment of smell and taste occurred on sertraline. There was an improvement when it was discontinued. This temporal relationship suggests that sertraline is the pathologic factor for smell and taste impairment in this individual. Serotonin (5-HT) fibers are prominent in olfactory bulb (Brunert et al 2016). Serotonergic neurons that innervate the olfactory bulb modulate the initial representation and processing of olfactory information between sensory neurons and cortex (Brunert et al 2016). 5-HT receptor agonists reduce odor evoked responses in the olfactory bulb (Baumgarten Von et al 1963; Bloom et al 1964; Petzold et al 2009). Increasing 5-HT attenuates odor-evoked synaptic input to glomeruli through 5-HT $2 \mathrm{C}$ receptors, which are intensified with decreased serotonergic activity (Petzold et al 2009). This supports the finding in this patient that 5-HT suppresses olfactory input. This occurs because sertraline causes inhibition of neuronal uptake of 5-HT (FDA, 2012) inducing relative 5-HT excess, thus inducing smell loss in this patient. The taste loss may similarly be due to increase in 5-HT. This is because taste is a physiological synesthesia, as retronasal smell. Retronasal smell is linked directly to about $90 \%$ of taste (Wahlstrom et al 2015). Thus, smell loss would also be perceived as taste loss. Query and assessment of olfactory and gustatory abilities in patients taking medications which enhance 5-HT are warranted.

\section{2}

Mixed effects modeling of placebo response across 8 placebo-controlled aripiprazole trials spanning 20 years in acutely relapsed schizophrenia patients

Alejandro Ochoa, PhD'; John D. Storey, PhD'; Srikanth Gottipati, PhD ${ }^{2}$; Shashank Rohatagi, PhD'; Andy Forbes, $\mathrm{PhD}^{2}$; Debbie Profit, PhD'; Raymond Sanchez, $M D^{2}$; Margaretta Nyilas, $M D^{2}$; and William Carson, $M D^{2}$

${ }^{1}$ Lewis-Sigler Institute for Integrative Genomics \& Center for Statistics and Machine Learning, Princeton University, Princeton, NJ, USA 
${ }^{2}$ Otsuka Pharmaceutical Development \& Commercialization, Inc., Princeton, NJ, USA

ABSTRACT: Study Objectives: The placebo response, or the response that is independent of the action of a drug, is a major confounder in drug efficacy trials for schizophrenia and other psychiatric disorders. Understanding the factors that control the placebo response could lead to improved study design and ultimately improved drug efficacy detection. Previous work has implicated demographic factors (gender, age, race, etc), time since start of trial, and study year, duration, and maximum dose with placebo response. Many studies have found increasing placebo response with study year.

METHOD: The placebo cohorts of 8 studies of varying design in which aripiprazole was evaluated for safety and efficacy in schizophrenia patients were analyzed. Response was quantified using the Positive And Negative Syndrome Scale (PANSS) in all studies. The data include longitudinal PANSS measurements for 846 individuals evaluated at 296 sites spanning 20 years. Overall, individuals were predominantly male $(72 \%)$, of Caucasian (47\%) or African American (42\%) self-reported race, and non-Hispanic (89\%). Ages ranged from 18 to 81, except for one pediatric study (ages 13-17). Linear mixed effects models were used to model response, with study and demographic variables treated as fixed effects while random baselines and slopes were modeled hierarchically by studies, sites, and individuals. Study identifiers: 31-93202; 31-97-201; 31-97-202 CN138-001; NCT00080327; NCT00102063; NCT00325689; NCT01663532

RESULTS: The placebo response is dominated by individual random effects $(\mathrm{p}=2.4 \mathrm{e}-35$ from a likelihood ratio test), but site random effects also play an important role $(p=9.8 \mathrm{e}-7)$. The number of weeks since the start of treatment is the largest significant fixed effect $(p<1 e-7$ using the Satterthwaite method), followed by a significantly different response for the pediatric study $(p=2.7 e-6)$ and for race "other" $(p=7.9 \mathrm{e}-5)$, which have small effect sizes. Response was not statistically significant for age $(p=0.10)$, gender $(p=0.71)$, or ethnicity (0.052). Study variables, such as year of study $(p=0.32)$, trial duration $(p=0.41)$, or maximum aripiprazole dose (0.038), did not pass the significance threshold at the 0.05 level after Bonferroni correction. Although study design has been the focus of much attention, here the small number of studies hinders statistical power to detect such associations. The inter-site variability is potentially explained by rater variability, but this information was unavailable.

CONCLUSIONS: Although the studies have varied designs, there are not enough studies to evaluate how their features affect the placebo response. Biases detected between sites suggest that improving rater reliability could reduce response variability. However, the placebo response may be largely controlled by unobserved variables that vary substantially across individuals, potentially including environmental or genetic factors.

FUNDING: Otsuka Pharmaceutical Development \& Commercialization, Inc.

103

Effect of adjunctive brexpiprazole on weight and metabolic parameters: an analysis of short- and long-term trials in major depressive disorder (MDD)

Hans Eriksson, $\mathrm{MD}^{\prime}$; Catherine Weiss, PhD ${ }^{2}$; Aleksandar Skuban, $\mathrm{MD}^{2}$; Peter Zhang, $P h D^{2}$; and Emmanuelle Weiller, PsyD ${ }^{\prime}$

${ }^{1}$ H. Lundbeck A/S, Valby, Denmark

${ }^{2}$ Otsuka Pharmaceutical Development \&

Commercialization, Inc., Princeton, NJ, USA

ABSTRACT: Study objectives: Brexpiprazole is a serotonindopamine activity modulator that is a partial agonist at 5-HT1A and dopamine D2 receptors, and an antagonist at 5-HT2A and norepinephrine $\alpha 1 \mathrm{~B} / 2 \mathrm{C}$ receptors, all at similar potencies. The short- and long-term effect of adjunctive brexpiprazole on weight and metabolic parameters in patients with MDD were evaluated based on pooled data from two pivotal studies and pooled data from two long-term, open-label trials.

METHODS: In the two similarly designed pivotal studies (NCT01360632; NCT01360645), patients with MDD and inadequate response to 1-3 antidepressant therapies (ADT) were enrolled and received single-blind ADT for 8 weeks. Patients with persistent inadequate response after this prospective phase were randomized to ADT + brexpiprazole (fixed dose $1 \mathrm{mg}, 2 \mathrm{mg}$, or $3 \mathrm{mg}$ ) or ADT + placebo for 6 weeks. The long-term studies were 26-week (NCT01360866; ongoing; data cut-off May 2015; fixed dose: $0.5,1,2$, and $3 \mathrm{mg}$ ) and 52-week (NCT01447576; flexible-dose: $0.25-3 \mathrm{mg}$ ) studies with adjunctive brexpiprazole, that included de novo patients, those who had completed one of the two pivotal studies, or patients completing a phase 2 study. Pooled analysis was performed on weight and fasting metabolic parameters in the short- (Week 6 of placebo-controlled) and long-term (Week 26 of the open label) studies, respectively.

RESULTS: In the short-term studies, mean change in weight from baseline to Week 6 was $1.4(\mathrm{~N}=215)$, $1.6(\mathrm{~N}=174), 1.6 \quad(\mathrm{~N}=210)$, and $0.3 \mathrm{~kg} \quad(\mathrm{~N}=383)$ for the $1 \mathrm{mg}, 2 \mathrm{mg}, 3 \mathrm{mg}$ ADT + brexpiprazole and 
ADT + placebo groups, respectively. In the long-term studies, the mean change in weight from baseline to Week 26 was $2.9 \mathrm{~kg}(\mathrm{~N}=1259)$. For fasting metabolic parameters, mean changes from baseline to last visit in the short-term studies (ADT + brexpiprazole 1, 2, and $3 \mathrm{mg}$ vs $\mathrm{ADT}+$ placebo) were: total cholesterol 0.70 , 2.60 , and $1.49 \mathrm{vs}-0.06 \mathrm{mg} / \mathrm{dL}$; high-density lipoprotein cholesterol (HDL) 1.12, 1.21, and 2.07 vs $0.56 \mathrm{mg} / \mathrm{dL}$; low-density lipoprotein cholesterol (LDL) -1.50, 1.37, and -0.77 vs $1.18 \mathrm{mg} / \mathrm{dL}$; triglycerides $3.21,-0.83$, and $2.20 \mathrm{vs} 2.27 \mathrm{mg} / \mathrm{dL}$; and glucose $-0.75,-0.40$, and 0.70 vs $0.92 \mathrm{mg} / \mathrm{dL}$. Mean changes from baseline to Week 26 in the long-term studies were: total cholesterol $2.40 \mathrm{mg} / \mathrm{dL} ; \quad$ HDL $-2.31 \mathrm{mg} / \mathrm{dL}$; LDL $3.57 \mathrm{mg} / \mathrm{dL}$; triglycerides $14.92 \mathrm{mg} / \mathrm{dL}$; and glucose $3.51 \mathrm{mg} / \mathrm{dL}$.

CONCLUSION: After adjunctive treatment with brexpiprazole in patients with MDD, the mean weight change was 1.4 to $1.6 \mathrm{~kg}$ in the 6 weeks studies and $2.9 \mathrm{~kg}$ at week 26 in the long-term studies, with small changes in lipid profiles and other metabolic parameters.

FUNDING: The studies were funded by H. Lundbeck A/S and Otsuka Pharmaceutical Development \& Commercialization, Inc. Abstract previously presented at American Psychiatric Association 169th Annual Meeting (APA 2016), Atlanta, GA, USA.

\section{4}

\section{Effect of brexpiprazole on weight and metabolic parameters: an analysis of short- and long-term trials in schizophrenia}

Hans Eriksson, $\mathrm{MD}^{\prime} ;$ Catherine Weiss, $P h D^{2}$; Mary Hobart, PhD ; John Ouyang, $P h D^{2}$; and Emmanuelle Weiller, PsyD ${ }^{\prime}$

${ }^{1}$ H. Lundbeck A/S, Valby, Denmark

${ }^{2}$ Otsuka Pharmaceutical Development \&

Commercialization, Inc., Princeton, NJ, USA

ABSTRACT: Study Objectives: Around one-third of new cases of diabetes in patients with schizophrenia were in patients exposed to olanzapine, risperidone, or quetiapine in a US Veterans Health Administration cohort. Brexpiprazole is a serotonin-dopamine activity modulator that is a partial agonist at 5-HT1A and dopamine D2 receptors, and an antagonist at 5-HT2A and norepinephrine $\alpha 1 \mathrm{~B} / 2 \mathrm{C}$ receptors, all at similar potencies. The effect of brexpiprazole on weight and metabolic parameters in patients with schizophrenia was analyzed based on data pooled from either two pivotal studies (NCT01396421; NCT01393613); or the open-label extension to these studies (NCT01397786; data cut-off May 2015), and a phase 2, open-label extension study (NCT01649557).
METHODS: In the two similarly designed pivotal studies, patients with acute schizophrenia were randomized to fixed once-daily doses of brexpiprazole $2 \mathrm{mg}, 4 \mathrm{mg}$, or placebo for 6 weeks (data from an additional treatment group included in each study $[0.25 \mathrm{mg}$ and $1.0 \mathrm{mg}]$ are not presented). The two long-term studies were open-label, 52-week, flexible-dose (1-4 mg and 1-6 mg once daily) studies with brexpiprazole that included de novo patients, those who completed one of the two pivotal studies, or had completed a phase 2 study. Pooled analysis on weight and fasting metabolic parameters, including glucose and lipid metabolism-related laboratory measurements at Week 6 of the placebo-controlled studies and at Week 26 of the open label studies, are reported.

RESULTS: In the short-term studies, mean change in weight from baseline to Week 6 was $1.7(\mathrm{~N}=253), 1.4$ $(\mathrm{N}=251)$, and $0.4 \mathrm{~kg}(\mathrm{~N}=227)$ for the $2 \mathrm{mg}, 4 \mathrm{mg}$ brexpiprazole, and placebo groups, respectively. In the long-term studies, the mean change in weight from baseline to Week 26 was $1.5 \mathrm{~kg}(\mathrm{~N}=485)$. For fasting metabolic parameters, mean changes from baseline to last visit in the short-term studies for brexpiprazole 2 and $4 \mathrm{mg}$ vs placebo were: total cholesterol 2.22 and $2.97 \mathrm{vs}$ $-3.21 \mathrm{mg} / \mathrm{dL}$; high-density lipoprotein cholesterol (HDL) 1.51 and 0.46 vs $-1.78 \mathrm{mg} / \mathrm{dL}$; low-density lipoprotein cholesterol (LDL) 0.36 and 2.35 vs $-1.82 \mathrm{mg} / \mathrm{dL}$; triglycerides -1.39 and 0.75 vs $0.38 \mathrm{mg} / \mathrm{dL}$; and glucose -0.23 and $1.64 \mathrm{vs} 0.42 \mathrm{mg} / \mathrm{dL}$. Mean changes in metabolic parameters from baseline to Week 26 in the long-term studies were: total cholesterol $2.97 \mathrm{mg} / \mathrm{dL}$; HDL $\quad 1.01 \mathrm{mg} / \mathrm{dL} ; \quad$ LDL $\quad 0.92 \mathrm{mg} / \mathrm{dL}$; triglycerides $4.00 \mathrm{mg} / \mathrm{dL}$; and glucose $3.11 \mathrm{mg} / \mathrm{dL}$.

CONCLUSION: The mean weight change after treatment with brexpiprazole in patients with schizophrenia was 1.4 to $1.7 \mathrm{~kg}$ in the 6 weeks studies and $1.5 \mathrm{~kg}$ at week 26 in the long-term studies, with small changes in lipid profiles and other metabolic parameters.

FUNDING: The studies were funded by $\mathrm{H}$. Lundbeck A/S and Otsuka Pharmaceutical Development \& Commercialization, Inc. Abstract previously presented at 5th Biennial Schizophrenia International Research Society (SIRS) Conference 2016, Florence, Italy.

\section{5}

\section{Dextromethorphan/quinidine improved symptoms of pseudobulbar affect irrespective of concomitant antidepressant use}

Andrea E. Formella, PharmD'; David N. Alexander ${ }^{2}$; Andrew J. Cutler ${ }^{3}$; Stephen D'Amico ; Flora M. Hammond $^{4}$; William Sauvé ${ }^{5}$; Richard D. Zorowitz, and Joao Siffert ${ }^{1 *}$ 
${ }^{1}$ Avanir Pharmaceuticals Inc., Aliso Viejo, CA, USA

${ }^{2}$ David Geffen School of Medicine at UCLA, Los Angeles, CA, USA

${ }^{3}$ Florida Clinical Research Center, LLC, Bradenton, FL, USA

${ }^{4}$ Cornerstone Medical Group, Franklin, TN, USA

${ }^{5}$ Indiana University School of Medicine, Indianapolis, IN, USA

${ }^{6}$ TMS NeuroHealth, Richard, VA, USA

${ }^{7}$ MedStar National Rehabilitation Network, Washington, DC, USA

*Former employee of Avanir Pharmaceuticals Inc., Aliso Viejo, CA, USA

ABSTRACT: Objectives: Pseudobulbar affect (PBA) can occur secondary to certain neurological diseases or brain injury. It is characterized by frequent, uncontrollable laughing/crying episodes that can be exaggerated or incongruent to mood or social context. PBA may be mistaken for, or occur comorbidly with, depression and many patients receive antidepressant therapy. Dextromethorphan/quinidine (DM/Q) is currently the only FDA-approved PBA treatment (US). This exploratory analysis evaluated outcomes from a DM/Q effectiveness trial for PBA stratified by antidepressant use at baseline (BL).

METHODS: PRISM II was an open-label trial enrolling persons with a clinical diagnosis of PBA secondary to dementia, stroke, or traumatic brain injury (TBI) and a Center for Neurologic Study Lability Scale (CNS-LS) Score $\geq 13$. All participants received DM/Q 20/10 mg BID for 90 days. Patients taking antidepressants were allowed to enroll provided doses were stable ( 2 months). Outcome measures evaluated included the CNS-LS score (primary), PBA episode counts, PBA episode quality of life impact (QoL-Visual Analog Scale), Patient Health Questionnaire (PHQ-9), and Mini-Mental State Examination (MMSE). Comparisons of LS mean change from BL to Day 90 for antidepressant users vs non-users were analyzed for each cohort (TBI, dementia, and stroke) with a 2-sample t-test using an ANCOVA model with BL score as a covariate. Safety data were analyzed descriptively.

RESULTS: A total of 367 patients enrolled, including 120, 113 , and 134, with TBI, stroke, and dementia, respectively. Of these, $48.5 \%[42.5 \%, 45.1 \%$, and $56.7 \%$, respectively] were using antidepressants. From baseline to Day 90/endpoint, significant improvements were observed for all outcome measures, including CNS-LS and PHQ-9, regardless of antidepressant use. Reductions in CNS-LS score were similar for antidepressant users vs non-users: LS mean change -8.2 vs $-7.1[\mathrm{P}=.08]$ and were similar within disease cohorts: TBI -8.7 vs $-8.4[\mathrm{P}=.72]$, stroke -8.5 vs $-6.8[\mathrm{P}=.13]$, and dementia
-7.6 vs $-6.7[\mathrm{P}=.36]$. Likewise, there were no significant differences in other outcome measures by antidepressant use, with the exception of MMSE score in the stroke cohort [improvement of 0.5 for antidepressant users vs 1.7 for nonusers, $\mathrm{P}=.02$ ]. The overall incidence of $\mathrm{AEs}$ was $37.6 \%$ for antidepressant users and $34.4 \%$ for nonusers. Adverse events occurring in $\geq 2 \%$ of overall patients were: diarrhea $5.4 \%$ (2.8\% antidepressant users vs $7.9 \%$ non-users), headache $4.1 \%$ (5.6\% vs $2.6 \%)$, dizziness $2.5 \%$ ( $1.7 \%$ vs $3.2 \%$ ), and UTI $2.7 \%$ ( $2.2 \%$ vs $3.2 \%$ ). Serious AEs were reported in $6.3 \%$ (5.6\% vs $6.9 \%$ ) and $9.8 \%$ (7.9\% vs $11.6 \%$ ) discontinued for AEs.

CONCLUSIONS: In this analysis, DM/Q was associated with PBA symptom improvement, and reduced depressive symptoms, regardless of concomitant $\mathrm{AD}$ use at $\mathrm{BL}$. These findings were observed across a range of disease subgroups associated with PBA.

\section{6}

\section{Delayed phantogeusia}

Antoine Sioufi, B.Sc ${ }^{1}$; and Alan Hirsch, $M D R^{2}$

${ }^{1}$ St. James School of Medicine, The Valley, Anguilla, B.W.I.

${ }^{2}$ Smell \& Taste Treatment and Research Center, Chicago, IL, USA

ABSTRACT: Introduction: Delay in development of phantogeusia after exposure to odors has not been reported.

METHODS: Case Report: A 57 year-old male presented with four years of myriad odors inducing dirhinous congestion followed 10 minutes to 2 hours later by a taste of the same odor, 4-7/10 in intensity, which would persist for 4-5 hours, despite leaving the site of odor exposure. Concurrent with the taste, he would frequently experience a throbbing headache associated with lacrimation and blurred vision.

RESULTS: Chemosensory Testing: Olfactory: Quick Smell Identification: 3 (normosmia). Pocket Smell: 3 (normosmia). Odor Memory: 10 seconds: 1; 30 Seconds: 0; 60 seconds: 1; total: 2 (anosmia). Alcohol Sniff: 23 (normosmia). Sniff Magnitude: 1.87 (anosmia). Suprathreshold Amyl Acetate Odor Intensity: crossed pattern (abnormal). Suprathreshold Amyl Acetate Hedonic: crossed pattern (abnormal). Olfactometer Identification: left 9, right 10 (hyposmia). Olfactometer Butanol Threshold: left 8 (normosmia), right 3 (anosmia). Sniff-N-Sticks Threshold: left $<1$, right $<1$, dirhinous $<1$ (anosomia); Dirhinous Discrimination: 8 (hyposmia); Dirhinous Identification: 6 (anosmia). Brief Smell Identification: 9 (normosmia). Retronasal Smell Index: 4 (reduced). Gustatory: Taste quadrant: hypogeusia anteriorly to all modalities and whole 
mouth taste weakness to sodium chloride. Threshold: normogeusia to sucrose, urea, and phenylthiocarbimol; mild hypogeusia to salt and hydrochloric acid. Electrogustometry: $>34$ on the + right posterior tongue, $>34$ left and right anterior tongue, left palate 12, right palate 14. Other: Fungiform Papillae: 22 right, 24 left (normal). MRI of brain with and without infusion: normal.

DISCUSSION: Reverberating discharges in the olfactory cortex could spread to integrated olfactory gustatory nuclei in the insula and from there to the primary gustatory cortex, inducing a phantogeusia, the chemosensory equivalent of the migraine visual spreading depressing of Leao. This may represent odor sensitive migraine with associated phantogeusia. A treatment approach may be administration during odor exposure, homologous to medications administered of medication during migraine aura.

107

Role of aripiprazole in the treatment of risperidone-induced hyperprolactinemiaa case report and literature review

Anupama Sundar, $\mathrm{MD}^{\prime}$; Ifeoluwa Osewa, $\mathrm{MD}^{\prime}$; Sesha Krishna Kotapati, $\mathrm{MD}^{\prime}$; and Sahil Munjal, $\mathrm{MD}^{\prime}$

${ }^{1}$ New York Medical College at Westchester Medical Valhalla, NY

ABSTRACT: Aripiprazole, which has a unique effect as an antipsychotic, is a D2 receptor partial agonist. It is an atypical antipsychotic with limited extrapyramidal symptoms. Since it acts as an antagonist in hyperdopaminergic conditions and as an agonist in hypodopaminergic conditions, it does not have adverse effects on serum prolactin levels. Risperidone is now commonly used as an atypical antipsychotic drug and has a high affinity for dopamine-D2 and 5-HT2 receptors. Prolactin levels increase in patients treated with risperidone, and this increase is higher than that seen with other secondgeneration antipsychotics. Menstrual disorders, galactorrhea, sexual dysfunction, gynecomastia, infertility, abnormal carbohydrate and lipid metabolism, and reduced bone density are some of the complications secondary to hyperprolactinemia. Many studies have demonstrated that adding aripiprazole to risperidone reduced prolactin levels in both men and women. Hyperprolactinemia has been observed in $45-100 \%$ of the patients treated with risperidone and $25 \%$ of them reported sexual side effects. We present a case of a 41-year-old male who developed significant risperidone induced hyperprolactinemia and we successfully treated the patient with aripiprazole. We will also review the literature on the role of aripiprazole and other strategies that can be used to target drug induced hyperprolactinemia in patients.

108

Twice weekly dTMS monotherapy is effective in maintaining acute-phase responders and improving the response rate for TRD patients

Aron Tendler, MD, C.BSM ${ }^{1,2}$; Abraham Zangen, $\mathrm{PhD}^{1,3}$; Mark George, $\mathrm{MD}^{4}$; and Yiftach Roth, $\mathrm{Ph} \mathrm{D}^{1,3}$

${ }^{1}$ Brainsway Jerusalem, Israel

${ }^{2}$ Advanced Mental Health Care Palm Beach, FL

${ }^{3}$ Department of Life Sciences, Ben-Gurion University, Beer Sheva, Israel

${ }^{4}$ Ralph H Johnson VA Medical Center, Department of Psychiatry and Behavioral Sciences, Medical University of South Carolina, Charleston, SC, USA

ABSTRACT: Introduction: Deep transcranial magnetic stimulation (dTMS) has been shown to be safe and effective for treatment resistant depression. Our goal was to analyze the efficacy of continuation dTMS monotherapy at twice a week for 12 weeks following the acute phase of four weeks of five times a week treatment in the active arm of the Brainsway multicenter double-blinded depression study. We assessed the probability of response over 16 weeks and the durability of clinical response among responders at the end of the 4-weeks acute phase, for whom the 12 week continuation phase is a maintenance phase.

METHODS: 89 unmedicated dTMS multicenter trial participants received the active treatment. 30 patients met responder criteria ( $\geq 50 \%$ decrease in HDRS-21) at the conclusion of the 4 weeks acute phase. The acute treatment phase consisted of 5-a-week H1-coil dTMS. Each 20 -min session consisted of $18 \mathrm{~Hz}$ dTMS delivered at $120 \%$ individual motor threshold, in 2-sec pulse trains separated by 20 -sec inter-train intervals, for a total of 55 trains, 1980 pulses. Continuation phase treatment consisted of twice-weekly dTMS for 12 weeks following the acute-phase. Patients and study personnel were blinded for the entire study. Kaplan-Meier survival curve (time to event [response]) was used to characterize outcomes during weeks 1 to 16 in the whole sample $(n=89)$ of active dTMS recipients. Percentage of time in response and in remission (HDRS-21<10) for each patient, and percentage of patients in response/ remission at each time point, were calculated for 4 -week responders during the continuation phase.

RESULTS: $59 / 89$ (66.3\%) achieved responder status at any time point. Of 23 subjects who did not respond earlier and continued at least 2 weeks into the continuation 
phase, 15/23 (65.2\%) achieved responder status during the continuation phase. Among the 30 responders at the end of week 4 , the vast majority showed sustained durability throughout their 12 weeks maintenance. $73.9 \% \pm 7.7 \%$ of patients were in responder status, and $59.8 \% \pm 8.4 \%$ of patients were in remission at each time point. Mean \pm SEM of $\%$ of time in response out of their total time in the twice weekly phase were $74.7 \% \pm 5.4 \%$, and of time in remission $59.7 \% \pm 7.1 \% .12 / 30$ patients $(40 \%)$ were $100 \%$ of their time in the twice weekly phase in response, and $9 / 30$ patients $(30 \%)$ were $100 \%$ of their time in remission. $24 / 30$ patients $(80 \%)$ were $60 \%$ or more of their time in response, and 18/30 patients $(60 \%)$ were $60 \%$ or more of their time in remission.

CONCLUSIONS: The vast majority of patients who continued twice weekly dTMS treatments reached response. The odds for a patient to reach response increased with the number of dTMS sessions, even for patients who did not respond for several weeks. Twice weekly dTMS maintenance monotherapy was efficacious for acute phase responders, with $80 \%$ of patients remaining in responder status for $60 \%$ or more of their time in the maintenance phase.

109

\section{Deep repetitive transcranial magnetic stimulation treatment of daily chronic cocaine users}

Aron Tendler, $M D^{1,2}$; Mark DeLuca, MD'; Elyssa Sisko, BA, CCRC'; Noelia Rodriguez, $\mathrm{MS}^{\prime}$; Sky CorbettMethot, BS, BA'; Jacqulynn Sutton-DeBord, BA, BS'; and Jeanette Brown, $B A^{\prime}$

${ }^{1}$ Advanced Mental Health Care Inc., Royal Palm Beach, FL, USA

${ }^{2}$ Brainsway Jerusalem, Israel

ABSTRACT: Cocaine has been abused for over 100 years, and we still don't have any effective treatments. In the United States, the 12-month prevalence amongst adults is $\mathbf{0 . 3 \%}$. Transcranial magnetic stimulation studies show decreased cortical excitability in cocaine dependent patients. Decreased activity in the brains reward circuitry is associated with cravings, relapse, and continued use. Deep repetitive transcranial magnetic stimulation (dTMS) can reach structures $4 \mathrm{~cm}$ beneath the dura and induce long lasting neuroplastic changes. We utilized the H4 dTMS coil to stimulate the bilateral prefrontal and insular cortices of three outpatients with chronic cocaine dependence that continued to relapse despite current best practice addiction treatments. After being shown drug specific cues, $10 \mathrm{HZ}$ dTMS was administered $6 \mathrm{~cm}$ anterior to the motor strip at $120 \%$ of the resting motor threshold (MT) of the hand, in 3 second stimulation pulses with a 15 second inter train interval for 1800 total pulses. Treatments were done for three weeks on a daily basis followed by twice-a-week maintenance. Cue related ravings decreased after the first treatment, and patients achieved sobriety by the second week of consecutive treatments. Potential mechanisms include replacing the drug stimulus with dTMS, correcting inhibitory control deficits with dTMS, and renormalizing the nucleus accumbens response to normal rewards via dTMS. A sham controlled randomized study of dTMS for cocaine or stimulant use disorder is warranted.

110

Waking up psychotic: case presentation of altered mental status persisting when awoken from nightmare

Carolyn Certo Gnerre, $\mathrm{MD}^{\prime}$; Esther Rollhause, $\mathrm{MD}^{\prime}$; and Howard L Forman, $M D^{\prime}$

${ }^{1}$ Montefiore Medical Bronx, NY

ABSTRACT: Objective: Review differential diagnosis for a patient with no psychiatric history who presents with symptoms of psychosis.

BACKGROUND: There is a broad differential diagnosis for patients with no significant past medical or psychiatric history who present with altered mental status. When a patient presents with psychosis from sleep, sleep-wake disorders must be added to the differential. The junction of neurological disease, psychiatric disease, and sleep-wake disorders can be a complicated arena to navigate and thus review is paramount to proper diagnosis.

CASE DESCRIPTION: A 28-year-old woman with no significant past medical or psychiatric history was brought in by her husband for bizarre behavior during and after being awoken from sleep for the second consecutive night. The patient was crying out during sleep, and due to her distress her husband woke her up. Though awake, patient continued to not respond directly to husband, speaking illogically, and sat staring out a window with a curtain wrapped around her head. On arrival to the ED, psychiatry was consulted and she required administration of psychotropic medication. Initial differential diagnosis was broad including organic causes such as neoplasm, meningitis, or metabolic disorder; neurologic disorder such as seizure; or psychiatric condition such as mania, first break psychosis, and REM sleep behavior disorder. After sleeping for several hours in the ED she awoke and returned to baseline. Neurology evaluation, including hematologic tests, lumbar puncture, EEG, and brain and spine imaging returned normal. Psychiatric condition was 
most likely. While an inpatient, the patient was followed by CL Psychiatric team, and was able to sleep without further incidents. During further psychiatric evaluation patient admitted to increased psychosocial stressors and restrictive eating habits. She had limited memory of the events, and reports that she had poor sleep and had not been eating well in the immediate days prior.

CONCLUSION: The limited episodic nature of the events and normal blood and imaging results led to high suspicion of primary psychological or psychiatric cause. Psychiatry and Neurology teams both assessed for most serious conditions and concluded that patient was safe for discharge with close follow up. She has followed up in the outpatient clinic regularly and the bizarre behavior has not recurred. Subsequent discussion with sleep disorder specialists offered no other possible diagnosis. This case is an illustration of the intersection between psychiatry and neurology, specifically in regards to sleep disturbances.

\section{1}

\section{Psychiatric stability in subjects with tardive} dyskinesia treated with valbenazine (NBI-98854)

Jean-Pierre Lindenmayer, $\mathrm{MD}^{\prime}$; Richard C. Josiassen, PhD ${ }^{2}$; Joshua Burke, $M S^{3}$; Scott Siegert, PharmD ${ }^{3}$; and Clinton Wright, PharmD ${ }^{3}$

${ }^{1}$ New York University School of Medicine

${ }^{2}$ Translational Neuroscience Research, Drexel

University College of Medicine

${ }^{3}$ Neurocrine Biosciences, San Diego, CA

ABSTRACT: Objectives: Tardive dyskinesia (TD) is a persistent movement disorder that is often induced by chronic exposure to dopamine receptor blocking agents (e.g., antipsychotics). Valbenazine (VBZ; NBI-98854) is a novel, highly selective vesicular monoamine transporter 2 (VMAT2) inhibitor currently in development for TD treatment. VBZ has exhibited a favorable efficacy and safety profile in multiple clinical studies including the KINECT 2 (NCT01733121) trial, a dose-escalating Phase 2 trial in which treatment with $\mathrm{VBZ}$ vs placebo (PBO) resulted in significant and clinically meaningful improvements in TD symptoms. The present analysis evaluated the psychiatric status of subjects in this study.

METHODS: KINECT 2 was a 6-week, randomized, doubleblind, PBO-controlled trial in subjects with schizophrenia, mood disorder, or gastrointestinal disorder and moderateto-severe TD. Subjects were randomized (1:1) to once-daily $\mathrm{PBO}$ or VBZ, which was initiated at $25 \mathrm{mg}$ and escalated in $25 \mathrm{mg}$ increments every 2 weeks to a maximum of $75 \mathrm{mg}$. The primary efficacy endpoint was defined as the change from baseline at Week 6 in the Abnormal Involuntary Movement Scale (AIMS) score for VBZ vs PBO, as scored by two blinded central raters. Safety assessments were analyzed descriptively and included the following psychiatric scales: Positive and Negative Syndrome Scale (PANSS), Calgary Depression Scale for Schizophrenia (CDSS), Young Mania Rating Scale (YMRS), Montgomery-Åsberg Depression Rating Scale (MADRS), and Columbia Suicide Severity Rating Scale (C-SSRS).

RESULTS: The safety population included $51 \mathrm{VBZ}$ and 49 PBO subjects; $76 \%$ of the VBZ subjects reached the maximum dose of $75 \mathrm{mg}$. Antipsychotics, antidepressants, and anxiolytics were the most common concomitant medications, taken by $\geq 40 \%$ of subjects in each group. The Week 6 change from baseline in AIMS score (primary endpoint) was significantly greater for VBZ vs PBO $(P=0.0005)$. Psychiatric status remained stable or improved from baseline in both groups, as shown by the following mean score changes at Week 6: PANSS Positive (VBZ -0.6, PBO -1.0), PANSS Negative (VBZ 0.5, PBO -0.9), and PANSS General Psychopathology (VBZ -0.5, PBO -0.7); CDSS (VBZ -0.9, PBO -0.7); YMRS (VBZ -1.1, PBO -0.3); and MADRS (VBZ -1.5, PBO -0.2). The percentage of subjects with suicidal ideation or behavior as measured by the C-SSRS for VBZ vs PBO was $5.9 \%$ vs $2.0 \%$ (screening) and $5.9 \%$ vs $0 \%$ (Weeks 2-8).

CONCLUSIONS: There was no apparent increase in psychopathology, depression, or suicidality with VBZ. Psychiatric status remained stable or improved in subjects with TD who had underlying schizophrenia, schizoaffective disorder, depression or bipolar disorder.

FUNDING: Neurocrine Biosciences, Inc.

\section{2}

\section{Disruptive mood dysregulation disorder: medical management without the use of antipsychotics}

Daniel T. Matthews, $M D^{\prime}$; and Glenda W. Matthews, $M D, P A^{\prime}$

${ }^{1}$ Universal Health Services, King of Prussia, PA

BACKGROUND: The new DSM-5 diagnosis of Disruptive Mood Dysregulation Disorder (DMDD) has sparse research available exploring medical management of the hallmark symptoms of persistent irritability and severe temper outbursts that occur, on average, three or more times per week over a period of one year. Several recently published studies resultant from applying the official DSM-5 diagnostic criteria to large community-based mental health treatment populations indicate 3 month prevalence rates of $3.3 \%$ to $8.2 \%$ with poor long-term functional prognosis, when treated with currently applied medication regimes.

OBJECTIVE: The current study explores the feasibility of more effectively managing DMDD symptoms with a unique medication protocol consisting of anticonvulsant 
and dopaminergic medications, without the use of antipsychotic medications.

METHODS: Subjects were 91 persistently irritable and explosive juveniles (52 mail, 39 female: age 6-17) with previous residential treatment for primary diagnosis of Bipolar, or other Mood Disorders, who met full criteria for DMDD upon retrospective chart review. All were discharged on oxcarbazepine (blood levels $=25-35 \mathrm{mcg}$ / $\mathrm{ml}$ ) and amantadine HCI (total daily dose of $10-15 \mathrm{mg}$ / $\mathrm{kg} /$ day) and no antipsychotic medication. Outpatient physicians were requested to be compliant with the treatment protocol; however some were non-compliant, adding or substituting antipsychotic medication.

RESULTS: The percent of re-hospitalization for uncontrolled aggression at 6 months post-discharge was calculated separately for those whose aftercare physicians were, or were not, fully compliant with the protocol. For the fully compliant, $\mathbf{8 \%}$ (5 of 64 ) required re-hospitalization. For those non-compliant, 26\% (7 of 27) required re-hospitalization. Using chi square analysis, there was a significant relationship between re-hospitalization rates and compliance (chi square, two tailed with Yates-3.975. $\mathrm{P}<.05 ; \mathrm{Phi}=.24)$.

CONCLUSION: The results indicate that, for youth 6 months post-discharge from residential treatment for DMDD, continuation of the above described medication protocol provides significantly lower re-hospitalization rates. This outcome suggests that further controlled studies are needed.

\section{3}

\section{Detox protocol for acute alcohol and drug withdrawal}

\section{David Michael Mathis, DO, FASAM, DABAM ${ }^{\prime}$}

${ }^{1}$ NaphCare, Birmingham, AL, USA

ABSTRACT: 15 Day Klonopin Taper: Klonopin $0.5 \mathrm{mg}$ po three times daily for 5 days, then decreases to, Klonopin $0.5 \mathrm{mg}$ twice a day for 5 days, then decrease to $0.5 \mathrm{mg}$ daily for 5 days, then stop. Consider using Klonopin $1 \mathrm{mg}$ 15 day taper for anticipated severe withdrawal such as: -History of several previous withdrawal episodes requiring detox -History of withdrawal seizures or delirium -Patients over age 40 with current acute medical conditions such as pneumonia, acute pancreatitis, cancer, and delirium

-History of recently using high doses of Xanax, IV opiate, or IV methamphetamines

Klonopin $1 \mathrm{mg} 15$ day 30 pills, Klonopin $1 \mathrm{mg}$ three times daily for 5 days, then decrease Klonopin to $1 \mathrm{mg}$ bid for 5 days, then decrease to Klonopin $1 \mathrm{mg}$ daily for 5 days.

TYPICAL CLINICAL DETOX PROGRESSION: Expect the Klonopin taper will control withdrawal symptoms within
24 to 48 hours. Prompt initiation of Klonopin will result in decrease of withdrawal symptoms easier and faster. If I initiate Klonopin in the afternoon or evening, I will order one or two doses of Klonopin on the first day, and begin the 15 day taper on the next morning. Completion of the 15 day taper is important for two reasons. It eliminates the risk of reemergence of withdrawal symptoms that can occur if Klonopin is abruptly stopped. It will decrease the frequently observed chronic symptoms of insomnia, anxiety, and irritability that addicted patients exhibit after the acute withdrawal phase. The patients will be more responsive to psychotropic medications to treat these symptoms. I prefer to use the term "post-acute withdrawal syndrome" to categorize those commonly reported symptoms after detox. Research on the neuroadaptations that occur in addiction include a release of CRF (corticotrophin releasing factor), which is a chronic stress response, that has been called "the dark side of addiction" by George Koob, Ph.D. In my opinion, "the dark side of addiction" presents clinically as this "post-acute withdrawal syndrome." Effective prompt detox allows the gaba-glutamate activity to try to approach its normal balance or homeostasis. However, it does not appear that this gabaglutamate activity reaches pre-addictive balance and is called in the addiction research literature as being in an allostatic state. This chronic stress response is felt to be the main driver for continued use of drugs in patients who suffer with addiction.

MEDICAL MANAGEMENT OF WITHDRAWAL: Fluid and electrolyte management will provide supportive treatment. Prompt correction of abnormalities, including hypokalemia and hypomagnesia, will decrease the risk of arrythymias and sudden death. Prompt initiation of treatment with Klonopin will indirectly decrease the hyperadrenergic response frequently seen in withdrawal, and may eliminate the need to use drugs such as clonidine.

\section{4}

\section{The effect of deuterium substitution on clinical} outcomes with deutetrabenazine in the First-HD and ARM-TD studies

Hubert H. Fernandez, $M D^{\prime}$; Karen E. Anderson, $M D^{2}$; David Stamler, $\mathrm{MD}^{3}$; Samuel Frank, $\mathrm{MD}^{4}$; and Claudia M. Testa, MD, $P h D^{5}$

${ }^{1}$ Cleveland Clinic, Center for Neurological Restoration, Cleveland, $\mathrm{OH}$, USA

${ }^{2}$ Georgetown University, Washington, DC, USA

${ }^{3}$ Teva Pharmaceuticals, La Jolla, CA, USA

${ }^{4}$ Harvard Medical School/Beth Israel Deaconess

Medical Center, Boston, MA, USA

${ }^{5}$ Virginia Commonwealth University, Richmond, VA, USA; on behalf of the ARM-TD and HSG/First-HD

Investigators 
ABSTRACT: Background: Deutetrabenazine (DTB) is a novel, highly selective vesicular monoamine transporter type 2 (VMAT2) inhibitor that contains deuterium, a naturally occurring, nontoxic form of hydrogen. Deuterium forms a stronger bond with carbon than does hydrogen, requiring more energy for cleavage. Deuterium attenuates metabolism and results in a unique pharmacokinetic profile that leads to more uniform systemic exposure without altering the target pharmacology of the original molecule. The resultant pharmacokinetic profile may enable less-frequent, lower daily doses, achieving adequate systemic exposure with lower peak concentration. Collectively, deuterium technology has the potential to improve the risk-benefit profile of medications.

OBJECTIVE: To assess whether DTB provides a clinical benefit for patients with chorea associated with Huntington disease (HD) in the First-HD study and tardive dyskinesia (TD) in the ARM-TD study.

METHODS: Patients with HD or TD were randomized 1:1 to DTB or placebo in the respective 12-week, double-blind trials, First-HD $(\mathrm{N}=90)$ and ARM-TD $(\mathrm{N}=117)$, which evaluated efficacy, safety, and tolerability. The primary efficacy endpoint of First-HD was the change from baseline to maintenance in the Unified Huntington's Disease Rating Scale (UHDRS) total maximal chorea (TMC) score. The primary efficacy endpoint of ARM-TD was the change in Abnormal Involuntary Movement Scale (AIMS) score from baseline to Week 12. Adverse events (AEs) and specific safety scales were monitored throughout both studies.

RESULTS: In First-HD, DTB significantly decreased the UHDRS TMC score compared with placebo (-4.4 vs -1.9; P?0.0001). In ARM-TD, DTB significantly reduced mean AIMS scores compared with placebo $(-3.0$ vs -1.6 ; $\mathrm{P}=0.0188$ ). DTB was generally well tolerated in both studies. In First-HD, AEs were mostly mild to moderate, and $\mathrm{AE}$ rates were similar for DTB and placebo; both groups also had the same low rates of dose reductions (6.7\% each), suspensions (2.2\% each), and withdrawals (2.2\% each) due to AEs. Likewise, the incidence of AEs in ARM-TD was also similar between DTB and placebo. These were also mostly mild to moderate in nature. For the DTB and placebo groups in ARM-TD, respectively, there were low rates of dose reductions (10.3\% vs $5.1 \%)$, suspensions $(5.2 \%$ vs $8.5 \%)$, and study withdrawals (1.7\% vs $3.4 \%)$ due to AEs.

CONCLUSIONS: The favorable efficacy and safety profile of DTB observed in the HD and TD populations in First-HD and ARM-TD may be attributed to the unique pharmacokinetic profile of DTB achieved by deuterium substitution.

FUNDING: These studies were funded by Teva Pharmaceutical Industries Ltd, Petach Tikva, Israel.
115

Hallermann-Streiff syndrome (HSS): a case report of HSS in psychiatric setting in the United States

Deepti Kaul Mughal, $\mathrm{MD}^{\prime}$; Nikhil D Nihalani, MD, $B C^{2}$; and Sanjay Mughal, $M D, B C^{3}$

${ }^{1}$ TMS Specialist, Nihalani Jacksonville, FL

${ }^{2}$ Psychiatry and Forensic Psychiatry, Nihalani Clinic, Jacksonville, FL, USA

${ }^{3}$ Internal Medicine and Geriatrics, Department of Hospital Medicine, University of Florida Health Sciences Center, Jacksonville, FL, USA

ABSTRACT: Hallermann-Streiff syndrome (HSS) is a very rare congenital occulomandibulodyscephaly mainly affecting the head and face. It was first reported in the medical literature by Aubry in 1893. This syndrome was named by two investigators that reported cases and established it as a distinct entity. Hallermann reported three cases in 1948 and then Streiff reported two cases in 1950. HSS occurs sporadically and there is no established pattern of inheritance. Currently only about 150 cases have been reported in medical literature. HSS is characterized by seven essential features as described as diagnostic criteria by Francois. These signs are dyscephalia and bird like faces, dental abnormalities, proportionate short stature, hypotrichosis, atrophy of the skin (especially the nose), bilateral microphthalmos, and congenital cataract. We report a case of 52-year-old Caucasian female with HSS with severe behavioral symptoms. Behavioral symptoms include motor hyperactivity, hand flailing and rocking back and forth, agitation and irritability, crying spells, occasional growling and barking sounds, and daytime sleepiness. Our patient had almost all seven symptoms of the diagnostic criteria. Mental and physical development were affected in our patient; she was fully dependent on a caregiver for activities of daily living since her birth. She also had severe oligophrenia. Association of HSS with behavioral problems has been reported in patients with oligophrenia in the literature. The patient was treated by psychotherapy and mood stabilizers. It is prudent that interdisciplinary approach should be used in managing these patients, and the role of behavioral management is crucial. It is one of the rare cases of HSS reported in psychiatric settings.

116

\section{Pharmacologic characterization of valbenazine (NBI-98854) and its metabolites: in vitro study results}

Dimitri E. Grigoriadis, PhD'; JD Evan Smith'; Ajay Madan, PhD ${ }^{2}$; Scott Siegert, PharmD'; Clinton Wright, PharmD ${ }^{\prime}$; and Haig Bozigian, $P h D^{\prime}$ 
${ }^{1}$ Neurocrine Biosciences Inc., San Diego, CA, USA

${ }^{2}$ Crinetics San Diego, CA

ABSTRACT: Objectives: Tardive dyskinesia is a persistent movement disorder induced by chronic neuroleptic exposure that leads to dopamine hypersensitivity. Modulation of presynaptic dopamine has long been hypothesized to mitigate these persistent involuntary movements. Valbenazine (NBI-98854), a novel compound that inhibits the vesicular monoamine transporter 2 (VMAT2), is in Phase 3 development for the treatment of tardive dyskinesia. VMAT2 is a presynaptic protein that regulates the packaging and subsequent release of dopamine and other monoamines from neuronal vesicles into the synapse. Valbenazine has 2 major metabolites: $(+) \alpha$-dihydrotetrabenazine (DHTBZ) and a mono-oxy metabolite (NBI-136110). Metabolites of tetrabenazine, another VMAT2 inhibitor, include $(+) \alpha$-DHTBZ, $(+) \beta-$ DHTBZ, (-) $\alpha$-DHTBZ, and (-) $\beta$-DHTBZ. In vitro studies were conducted to characterize the pharmacologic characteristics of valbenazine and its metabolites, compared with those of the 4 tetrabenazine metabolites.

METHODS: Radioligand binding studies of valbenazine and its 2 major metabolites were conducted to assess their ability to inhibit binding of $\left[{ }^{3} \mathrm{H}\right]-\mathrm{DHTBZ}$ in both cells heterologously expressing VMAT2 and in native tissues that endogenously express the VMAT2 protein. The ability of these molecules to inhibit functional uptake of dopamine or serotonin via the transporter was also evaluated. In addition, a broad panel screen was conducted to test valbenazine, $(+) \alpha-$ DHTBZ, $(+)$ $\beta$-DHTBZ, (-) $\alpha$-DHTBZ, and (-) $\beta$-DHTBZ for off-target interactions at over 80 receptor, transporter, and ion channel sites.

RESULTS: Radioligand binding studies showed that $(+)$ $\alpha$-DHTBZ was a potent inhibitor of $\left[{ }^{3} \mathrm{H}\right]-\mathrm{DHTBZ}$ binding in homogenates of rat striatum $\left(\mathrm{K}_{\mathrm{i}}=1.0\right.$ to $\left.2.8 \mathrm{nM}\right)$, rat forebrain $\left(\mathrm{K}_{\mathrm{i}}=4.2 \mathrm{nM}\right)$, and human platelets $\left(\mathrm{K}_{\mathrm{i}}=2.6\right.$ to $3.3 \mathrm{nM})$. Results for valbenazine $(\mathrm{Ki}=110$ to $190 \mathrm{nM})$ and its NBI-136110 metabolite $\left(\mathrm{K}_{\mathrm{i}}=160\right.$ to $\left.220 \mathrm{nM}\right)$ showed that these compounds also exhibited an inhibitory effect on VMAT2, but to a lesser degree than $(+)$ $\alpha$-DHTBZ. Broad panel screening indicated that neither valbenazine nor its $(+) \alpha$-DHTBZ metabolite had any interactions at other protein targets including GPCRs, enzymes, and ion channels. In contrast, 2 of the tetrabenazine DHTBZ metabolites [(-) $\alpha$-DHTBZ and (-) $\beta$-DHTBZ] were relatively less potent at VMAT2 and demonstrated significant binding to serotonin $\left(5-\mathrm{HT}_{1 \mathrm{~A}}\right.$, $5-\mathrm{HT}_{2 \mathrm{~B}}$ and $\left.5-\mathrm{HT}_{7}\right)$ and/or dopamine $\left(\mathrm{D}_{2}\right)$ receptor sites.

CONCLUSIONS: Results from these in vitro studies indicate that $\left(^{+}\right) \alpha$-DHTBZ inhibits VMAT2 in a highly selective and specific manner. Neither valbenazine nor $(+)$
$\alpha$-DHTBZ had notable interactions at serotonin or dopamine receptors, suggesting the potential for fewer unwanted side effects mediated by these synaptic mechanisms. The pharmacologic characteristics of valbenazine and $(+) \alpha$-DHTBZ (i.e., VMAT2 inhibition, minimal off-target interactions) were consistent with the favorable efficacy, safety, and tolerability results observed in recent clinical studies.

FUNDING: Neurocrine Biosciences

\section{7}

The invisible disco machine-musical hallucinations in a patient with paranoid psychosis - case report and brief literature review

\section{Eduardo J. Rodriguez-Perez, $M D^{\prime}$; and Gerard C. Addonizio, $M D^{2,3}$ \\ ${ }^{1}$ Department of Psychiatry, Lincoln Medical Center, Bronx, NY, USA \\ ${ }^{2}$ Weill Cornell Medicine, New York, NY, USA \\ ${ }^{3}$ New York Presbyterian Hospital Westchester Division, White Plains, NY, USA}

ABSTRACT: Introduction: Musical hallucinations are a rare subtype of auditory hallucinations not strictly associated with psychiatric illness. Here we present a case of musical hallucinations in a patient with paranoid psychosis, successfully treated with the antipsychotic olanzapine. We review the etiologies, approaches to diagnosis, and treatment modalities of musical hallucinations.

CASE REPORT: Patient is a 67-year-old Caucasian man, with alcohol use disorder in sustained remission and with unilateral hearing loss, admitted to inpatient psychiatry for paranoid psychosis. During treatment, the patient reported the onset of what sounded like a radio coming from a neighbor's apartment, which predated paranoia by 3 months. He described a repeating rhythm of disco music, which he believed emanated from a machine located somewhere in the building, and was being played to upset him. He occasionally described a trumpet playing. Memory was grossly normal. The patient was managed with olanzapine, with good effect. Vocal auditory hallucinations resolved first, while musical hallucinations faded and remitted approximately one week later.

DISCUSSION: Auditory hallucinations (AH) include auditory perceptions in the absence of external auditory stimuli. AH most frequently are equated with vocal auditory hallucinations (VAH). AH also include tinnitus, comprised of simple sounds, and musical hallucinations, more complex perceptions of musical notes or melodies. 
MH are rare. Prevalence has been reported $0.8 \%$ to $0.16 \%$. Incidence of $\mathrm{MH}$ ranges from $0.125-4 \%$, depending on the study populations. It occurs in less than $1 \%$ of patients with hearing loss. The greatest proportion of MH (39\%) had a primary psychiatric illness, while $25 \%$ had a neurological disorder, drug toxicity/withdrawal (12\%), and structural CNS disease (9\%). MH have been associated with advanced age, degree of hearing loss, female gender, and presence of a mood disorder. Functional imaging studies have shown that brain structures involved in perception of actual sound are also activated during hallucinatory activity. Models hypothesized to explain MH include the Deafferentiation Model and the Perceptual Release Model. MH does not always necessitate treatment. In cases where hearing loss is the primary cause, remediation of hearing loss is the target. Psychotherapeutic approaches can be offered to patients not distressed by their MH. Pharmacologic treatment of persistent $\mathrm{MH}$ has included anticonvulsants, antipsychotics, and acetylcholinesterase inhibitors. There are reports of resolution of hallucinations after ECT.

CONCLUSIONS: Here we discussed the rare case of a patient with musical hallucinations and paranoid psychosis. A multidisciplinary approach to the assessment of MH can help determine etiology and guide treatment. Subtle changes in screening during psychiatric evaluations may increase the detection of $\mathrm{MH}$.

FUNDING: No funding.

\section{8}

\section{Psychosis in a patient receiving malaria chemoprophylaxis-case report and brief literature review}

Eduardo J. Rodriguez-Perez, $M D^{\prime}$; and Joseph Yuen, $M D^{\prime}$

${ }^{1}$ Department of Psychiatry, Lincoln Medical Center, Bronx, NY, USA

ABSTRACT: Introduction: Antimalarial treatments are known to cause a variety of neuropsychiatric side effects. Here we present a case of acute psychosis that began after a prophylactic course of mefloquine. We review the literature regarding psychiatric symptoms of antimalarial treatment, pathophysiology, and possible treatment approaches.

CASE REPORT: Patient was a 20-year-old African-American woman who presented floridly psychotic after returning from a family vacation to Africa. The patient had no past medical or psychiatric history. There was no family psychiatric history. Brain CT was normal. Family reported that the patient had completed a prophylactic course of mefloquine the previous week. Patient was started on risperidone, which had little effect. She was transitioned to asenapine, with modest effect. Patient was discharged home. Subsequent collateral reported the patient was doing well and had resumed her college coursework.

DISCUSSION: Mefloquine hydrochloride is a structural analog of quinine. Mefloquine is FDA indicated for the treatment of acute malaria infections, as well as prophylaxis. Half-life is 2-6 weeks. Weekly prophylactic doses produce a steady state concentration in 7 weeks. Metabolism is via CYP3A4. Synthetic quinolones have been shown to cause direct neuronal injury in both humans and animals, in a dose-dependent form. Specifically these cause neuronal degeneration in the brainstem, pontine, and vestibular nuclei. Testing of high-dose Mefloquine in rats showed permanent injury. A wide range of neuropsychiatric symptoms have been reported with mefloquine, and occur in 1/13,000 patients treated prophylactically, and 4-7/1000 patients with acute malaria. Three reported cases of psychosis were documented out of 16,491 reports of adverse events, or $0.018 \%$. Risk factors for psychiatric side effects include previous psychiatric history and past exposure to antimalarials. Some reports have found CYP deficiencies can lead to increased severity of side effects. In vitro studies using recombinant cell lines show that mefloquine acts as a full agonist at $5 \mathrm{HT} 2 \mathrm{c}$ receptors and partial agonist at 5HT2a receptors. Mefloquine has low D3 activity, and essentially no activity at D1, D2, or D4 receptors.

CONCLUSIONS: Here we discussed a rare case of a patient with acute onset of florid paranoid psychosis after completion of a prophylactic course of mefloquine. The patient's presentation is consistent with a DSM 5 diagnosis of Medication-Induced Psychotic Disorder. A treatment approach for this scenario could include management with an antipsychotic with primarily 5HT2c activity, such as asenapine, quetiapine, or olanzapine. Our case illustrates the importance of a thorough medication/ travel history when evaluating a first-episode psychosis. Additionally, use of mefloquine should be avoided in patients with any psychiatric history.

FUNDING: No funding.

\section{9}

\section{Comorbid generalized anxiety disorder (GAD) with adult attention deficit hyperactivity disorder (ADHD)}

Faruk S. Abuzzahab, Sr., MD, PhD'; Kathryn B. Abuzzahab, RN, JD, LLM ${ }^{1,2}$; and Ferial Kamsheh ${ }^{1,2}$

${ }^{1}$ Clinical Psychopharmacology Consultants, Minneapolis, MN, USA

${ }^{2}$ Augsburg College, Minneapolis, MN, USA 
ABSTRACT: Study Objectives: Although there are publications documenting the comorbidity of GAD with $\mathrm{ADHD}$, some concerns have been expressed about the administration of anxiolytics, mainly benzodiazepines, to adults with $\mathrm{ADHD}$ receiving central nervous system stimulants.

METHODS: A chart review was conducted on 50 outpatients who completed the adult ADHD-RS-IV with adult prompts scale and the GAD-7 scale. We divided the total sample of 50 outpatients into two groups: one group including patients who were taking anti-anxiety medications, and another group including patients who were not taking anti-anxiety medications.

RESULTS: The fifty adults with ADHD ranged in age between 18 and 71 with 29 males and 21 females. There was an average age of 43 years a 12.92 and an average body mass index of $28 \mathrm{~kg} / \mathrm{m}^{2}$ a 4.97 . The female subgroup ranged in age between 27 and 71, while the male subgroup ranged in age between 18 and 59 . The female subgroup had an average BMI of 28.5 a 4.75 and the male subgroup had an average BMI of 28.3 ą 5.21 . There was no significant difference in age or BMI for the female and male subgroups. The average ADHD score for the female subgroup was 35.48 a 12.05 and the average ADHD score for the male subgroup was 35.17 a 12.60 . The average GAD-7 score for the female subgroup was 16.23 a 4.65 and the average GAD-7 score for the male subgroup was 16.98 a 3.98 . There were no significant differences in $\mathrm{ADHD}$ or GAD-7 scores between the female and male subgroups. However, the 26 patients who were taking anti-anxiety medications had an average anxiety score of 18.88 a 2.91 , which is significantly higher than the 24 patients who were not taking anti-anxiety medications, whose average anxiety score was 13.7 ą 3.99 .

CONCLUSION: GAD was found to be comorbid with adult $\mathrm{ADHD}$ as determined by this GAD-7 scale. The adult ADHD patients who required anxiolytics had a higher GAD-7 score than the patients who did not need any additional medications for their anxiety.

FUNDING: Sponsored in part by the Psychopharmacology Fund and University of Minnesota Medical Foundation, Minneapolis, MN.

\section{0}

\section{Position-dependent palinosmia and phantigeusia}

Hardeep Singh Garcha'; and Alan Richard Hirsch, $M D^{2}$

${ }^{1}$ Windsor University School of Medicine, Saint Mary Cayon, St. Kitts \& Nevis

${ }^{2}$ Smell \& Taste Treatment and Research Foundation, Chicago, IL, USA

INTRODUCTION: Effects of body position on palinosmia and phantogeusia have not heretofore been described.
METHODS: Case study: A 60-year-old right handed male presented with gradual onset of chemosensory complaints of idiopathic origin including phantosmia, palinosmia, cacosmia, subjective hyperosmia, and myriad odor induced conditions including headaches. With exposure to odors he would develop phantogeusia coincident with the scent. After leaving the site of the aroma, the odor and the taste would persist. This taste would involve the entire mouth and lips and would last all day. However, upon lying down the intensity of the smell and taste would decline from 8-9/10 to $4 / 10$ in severity. This lowered level of intensity would persist for 4-5 hours, whereupon with dormancy, would dissipate. If awakened before sleeping 4 hours, the very same smell and taste would return to the initial intensity level of 8-9/10.

RESULTS: Chemosensory Testing: Olfaction: Quick Smell Identification: 3 (normosmia). Pocket Smell: 3 (normosmia). Odor Memory Test: 1 at 10 seconds, 0 at 30 seconds, 1 at 60 seconds; Total: 2/12 (anosmia). Alcohol Sniff: 23 (normosmia). Sniff Magnitude Ratio: 1.87 (anosmia). Suprathreshold Amyl Acetate Odor Intensity: crossed pattern (abnormal). Suprathreshold Amyl Acetate Odor Hedonic: rossed pattern (abnormal). Olfactometer Identification Test: L 9.0, R 10.0 (hyposmia). Olfactometer Butanol Threshold: L 8.0 (normosmia), R 3.5 (anosmia). Sniff-n-Sticks: Threshold: L $<1$, $\mathrm{R}<1$, Dirhinous $<1$ (anosmia), dirhinous discrimination: 8 (hyposmia), dirhinous identification: 6 (anosmia). Brief Smell Identification: 9 (normosmia). Retronasal Smell Index: 4 (abnormal). Gustation: Taste Threshold Testing: normogeusic to urea, sucrose, phenylthiocarbamide; mild hypogeusia of $10-30 \%$ to sodium chloride and hydrochloric acid. Taste Quadrant: decreased anterior tongue bilaterally to sodium chloride, sucrose, citric acid, quinine hydrochloride, and alcohol. Whole mouth weakness to sodium chloride. CT scan of brain and sinuses: normal.

DISCUSSION: If palinosmia represents pathological reverberating circuits of olfactory discharge, increase olfactory threshold with assuming the supine position may be of such magnitude as to render such reverberations subthreshold, thus breaking the circuit and eliminating the palinosmia. Lying down may function as a vestibular, cutaneous, proprioceptive, and kinesthetic distractor, thus changing the focus of attention from the chemosensory phenomenon to the change in position. Alternatively, lying down functions to decrease cortisol, and since cortisol enhances olfactory ability, reduction of this may reduce pathological olfactory function including palinosmia (Weitzman, Zimmerman, Czeisler, Ronda, 1983). Query as to positional dependency in those with palinosmia, phantosmia, and phantogeusia is warranted. 


\section{1}

\section{Home-based delivery for extended-release naltrexone in youth with opioid misuse}

Hoa T. Vo, PhD'; Ilya Rozenberg, MS, CRNP'; Rachel Burgower, $\mathrm{MS}^{3}$; and Marc Fishman, $\mathrm{MD}^{4}$

\author{
${ }^{1}$ Mount Manor Treatment Center Research Program \\ Baltimore, MD \\ ${ }^{2}$ University of Maryland School of Baltimore, MD \\ ${ }^{3}$ Research Coordinator \\ ${ }^{4}$ Medical Director at Mount Manor Treatment \\ Baltimore, MD
}

ABSTRACT: Objective: To present a small case series on a pilot program for nine patients enrolled in adjunctive home-based delivery of Vivitrol. Preliminary results of outcomes such as the number of doses, weekly urine toxicity results, and weeks retained in treatment were evaluated for each patient.

METHOD: Patients (ages 16-25 years) who received an initial dose of Vivitrol prior to discharge from an urban residential substance abuse treatment program were offered the option to receive subsequent doses of Vivitrol in-home. Doses were tracked and contact was made for patients' subsequent doses. Patients continued to receive in-clinic weekly psychosocial interventions, outpatient services for SUD (usual group format), and medication management. The primary outcomes were the patients' duration of abstinence from opioids, displayed by a continuously negative drug screen, and also the number of doses patients received. Written consent was obtained in order to participate in the pilot program.

RESULTS: We evaluated the 9 patients enrolled into the home-based delivery program and found variation in the number of doses received, retention in treatment length of time, and weekly urine toxicity results for opiate and other substances reported. The average number of Vivitrol doses administered in this small case series for those who received a home-based dose was 7.5 . Approximately $66 \%$ of those signed-up or enrolled in this program actually received a home-based dose. All those who received home-based doses remained abstinent from opioids throughout the duration of the program as evidenced by negative drug screens.

CONCLUSIONS: Opioid abuse and dependence continues to plague communities across the U.S. Traditional treatment models appear to be inefficient. While these numbers are small, this case series demonstrates the feasibility of home-based models for opioid use disorders. The average number of doses administered in $66 \%$ of the patients who initiated home-base care is high compared to doses administered in clinic through usual care (averaging between 2-4). Additional effort may be warranted in order to pilot such programs to enhance MAT delivery systems for youths with opioid addiction. The multi-faceted approach involving medication administration, therapy, and continuous monitoring would address the complexity of drug addiction, increasing motivation for treatment.

FUNDING: The staffs working on this clinical pilot program are employees and trainees of Mountain Manor Treatment Centers.

122

Transformation of burning mouth syndrome into burning lip syndrome

\author{
Jaspreet Singh ${ }^{\prime}$; and Alan R. Hirsch ${ }^{2}$ \\ ${ }^{1}$ Caribbean Medical University, Willemstad, Curacao \\ ${ }^{2}$ Smell and Taste Treatment and Research Foundation, \\ Chicago, IL, USA
}

ABSTRACT: Introduction: Burning mouth syndrome (BMS) frequently extends from the mouth and tongue into the lips (Getchell, 1991). Burning lip syndrome (BLS) involves burning of the lip without involvement of the tongue (Lamey, 1994). Transformation of BMS into BLS has not heretofore been described.

CASE REPORT: A 53-year-old right handed female presented with progressively severe BMS of $11 / 2$ years duration. Ambient aroma would induce a foul smell immediately followed by the taste of the odor and then burning of the tongue and mouth. The burning involved the tongue, tip of the tongue, back of the throat, palate and upper and lower lips which spread to the outside of her lips. After daily treatment with $0.5 \mathrm{mg}$ haloperidol for two months, her symptoms changed, migrating from her lips and tongue to lips alone. The burning involves the lower more than upper lip, in a perioral circular pattern, but only the external lips. This burning was precipitated by strong odors and lasts all day and is 4-5/10 in severity.

RESULTS: Chemosensory Testing: Olfactory Testing: Brief Smell Identification Test: 12 (normosmia) Alcohol Sniff Test: 8 (hyposmia). Retronasal Smell Testing: Retronasal Smell Index: 9 (normal). Gustatory Testing: Propylthiouracil Disc Taste Test: 9 (normogeusia). Neuropsychiatric Testing: Clock Drawing Test: 3 (abnormal). Animal Fluency Test: 12 (abnormal). Center for Neurologic Study-Lability Scale: 12 (abnormal). Tongue Candida Cultures: negative X2.

DISCUSSION: There are myriad possible mechanisms whereby BMS transforms into BLS. One model of BMS is as an oral variant of reflex sympathetic dystrophy. In this, the burning pain originates in the tongue and spreads to the lips. Treatment which reduces pain in the tongue would not necessarily reduce the associated 
lip pain. This could be induced by topical agents (Hirsch, 2009), or through counter-stimulation with taste, since taste inhibits pain (Hirsch, 2011). Thus any agents which enhance taste may also act to reduce the tongue pain. Haloperidol may have acted to induce a dry mouth and associated bitter taste (Mayo Clinic, 2016). This bitter taste may have functioned to inhibit mouth pain, without affecting the lip pain. An alternative construct of BMS is as a form of restless leg syndrome (Batta, 2016). Haloperidol may have induced orofacial lingual dyskinesia, causing tongue movement which thus acted to reduce BMS. Such tongue movement would be ineffective toward relieving BLS since the lips remain stationary. Another model for BMS involves worsening or exacerbation by ambient aromas (Hirsch, 2004), such was seen in this patient. Haloperidol may have acted to reduce olfactory ability. Such olfactory inhibition may have thus acted to prevent the odor induced phantogeusia and subsequent BMS. However, since the lips lack taste receptors, they would be unaffected by this inhibition in taste, allowing the pain to persist. Further investigation as to the transformation of BMS into BLS is warranted.

\section{3}

\section{Eating what lives in the pineapple under the sea: chemosensory loss from consumption of phytoplankton}

Jaspreet Singh' ${ }^{\prime}$ B Batta Priya ${ }^{2}$; Akshay U. Pawar ${ }^{3}$; and Alan R. Hirsch ${ }^{4}$

${ }^{1}$ Caribbean Medical University, Williemstad, Curacao

${ }^{2}$ Windsor University School of Medicine, Basseterre, St Kitts

${ }^{3}$ St. George's University School of Medicine,

St. George's, Grenada

${ }^{4}$ Smell and Taste Treatment and Research Foundation,

Chicago, IL, USA

ABSTRACT: Introduction: Impact of direct consumption of phytoplankton on the chemosensory system has not heretofore been reported.

METHODS: Case Study: Two years ago, a 53-year-old male sustained head trauma with loss of consciousness, followed by loss of smell and taste. There was gradual recovery, such that his sense of smell returned to $85 \%$ of normal. He denied dysosmia, cacosmia, phantosmia, and palinosmia. He affirmed flavorful eructations. Coincident, with smell loss, he noted an inability to taste. As smell returned, taste also gradually improved to $90 \%$ of normal, whereby he was able to taste everything, just of less intensity. He denied dysgeusia, cacogeusia, phantageusia, or palinogeusia. He began treatment with "Oceans Alive Pure Phytoplankton," a mixture of Nannochloropsis gaditana, Nannochloropsis galasemis, and concentrated purified sea mineral trace elements.
Before beginning the medicine, he observed his smell was $85 \%$ and taste was $90 \%$ of normal. Approximately 5 hours after taking 15 drops, his smell defervesced to $10 \%$ and taste $15 \%$ of normal, which remained poor for three days and then recovered to its baseline. With recurrent use, similar symptoms reappeared.

RESULTS: Chemosensory Testing: Olfactory: Brief Smell Identification Test: 11 (normosmia). Alcohol Sniff: 10 (hyposmia). Retronasal Smell Index: 1 (abnormal). Gustatory: Propylthiouracil Disc: 9 (normogeusia).

DISCUSSION: The strong temporal correlation between symptoms and chemosensory manifestations along with recurrence upon repeated challenges, strongly suggest a causal connection. The neurological effects in humans as a result of consuming fish that ingested phytoplankton may be due to its produced toxins: domoic acid, alpha-anatoxin, clupeotoxin, and okadaic acid (Grant 2010; Al-Sammak 2014; Chamberlain 2001; Kamat 2013). Physiologically, domoic acid may act on the glutamate receptors on the olfactory bulb, manifesting as a transient hyposmia (Lefebvre 2010). Alpha-anatoxins may function by stimulating nicotinic receptors in the olfactory bulb (D'souza 2013) or nicotinic receptors on the tongue, resulting in the chemosensory symptoms (Ren 2015). Alternatively the toxin could be reducing taste through a direct effect on glutamate receptors on the taste buds of the fungiform papillae (Yasuo 2008). On the other hand, domoic acid and okadaic acid may attack the hippocampus, reducing the ability to access chemosensory memories and thus identification (Pulido 2008; Kamat 2013), although this is less likely since phytoplankton toxins induces anterograde as opposed to retrograde memory (Pulido 2008). Given the normal taste test, another possibility is that the phytoplankton acted to impair olfaction with reduced perception of taste due to impaired retronasal smell (Gruss, 2015). Further investigation as to the effects of ingesting phytoplankton on chemosensation is warranted.

\section{4}

\section{Serum drug levels of medications without well-defined therapeutic windows: a case and therapeutic approach}

Jonathan M. Wai, MD ${ }^{1,2}$; Christopher Aloezos, $\mathrm{MD}^{1,3}$; and Margarita Kats, $\mathrm{MD}^{1,3}$

${ }^{1}$ Department of Psychiatry and Behavioral Sciences, Montefiore Medical Bronx, NY

2 The University Hospital for Albert Einstein College of Bronx, NY

${ }^{3}$ The University Hospital for Albert Einstein College of Bronx, NY 
ABSTRACT: Introduction: Monitoring of therapeutic drug levels is a mainstay of treatment for medications with well-defined therapeutic windows and severe toxicities that occur when levels are too high. However, most psychiatric medications have ill-defined therapeutic windows that make interpretation of laboratory results difficult. We present a case where serum drug levels were measured and propose an approach to guide the use of serum drug levels with ill-defined therapeutic windows.

CASE PRESENTATION: The patient is a 42 -year-old male with a psychiatric history of major depressive disorder, panic disorder, agoraphobia, post-traumatic stress disorder, and avoidant personality disorder currently taking lamotrigine, sertraline, and clonazepam who has been in outpatient treatment for six years after a 20-year period of untreated depression and anxiety. Due to his continued difficulties in social and occupational functioning while taking sertraline $200 \mathrm{mg}$ PO daily, a serum level of sertraline and desmethylsertraline was obtained. Results showed a sertraline level of $97 \mathrm{ng} / \mathrm{mL}$ and a desmethylsertraline level of $190 \mathrm{ng} / \mathrm{mL}$, both slightly above the reference range. Using the proposed algorithm, the clinician continued this dose of sertraline and augmented treatment with more intensive psychotherapy, including more frequent individual sessions and group work. Since this augmentation, the patient has shown an overall improvement in symptoms and social functioning.

DISCUSSION: This case report demonstrates how a simple algorithmic approach can help guide laboratory usage. Serum drug levels for medications without well-defined therapeutic windows should only be drawn when a patient is at a maximum approved dose of a medication. If the serum level is unusually low, then the clinician can consider increasing the dose, or perform a more thorough evaluation for adherence. When levels are within the normal range, one can consider increasing the dose or switching the medication. If the level is high, then the medication should generally not be further increased, instead switching the medication or augmenting if there is some improvement. Because of this patient's high serum drug level, the patient's sertraline dose was kept the same and therapy was augmented by more intensive psychotherapy. The blood level proved to be helpful as it provided the clinician with evidence that non-remission of symptoms occurred in the setting of treatment adherence and an adequate dose of the medication.

CONCLUSIONS: Interpretation of serum drug levels for medications with ill-defined therapeutic windows can be difficult since there are no standard practices for this. We propose a simple algorithm that may help guide the usage of serum drug levels and prevent unnecessary monitoring when the results will likely not be helpful.

\section{5}

\section{Binge eating disorder management: can medical education improve physician knowledge?}

\author{
Jovana Lubarda, PhD'; Piyali Chatterjee'; and \\ Leslie Citrome, $M D, M P H^{2}$ \\ ${ }^{1}$ Medscape Education, New York, NY, USA \\ ${ }^{2}$ New York Medical College, Valhalla, NY, USA
}

ABSTRACT: Study objectives: Binge eating disorder (BED) is the most prevalent eating disorder in the US, affecting approximately $2 \%$ of individuals at some point during their lives. However, despite this disorder's high prevalence and considerable impact on health and quality of life, many individuals with BED go undiagnosed and are treated suboptimally. Because BED was not included in the Diagnostic and Statistical Manual of Mental Disorders (DSM) as a distinct entity until 2013 and the first medication for BED was not approved until 2015, many clinicians are unfamiliar with criteria for diagnosing the disorder and tailoring evidence-based treatment options. This study assessed whether online continuing medical education (CME) in the format of a case-based challenge can improve clinically relevant knowledge and competence of psychiatrists and primary care physicians (PCPs) who manage patients with BED.

METHODS: The online CME activity, designed in an interactive case-based format consisting of 2 cases of BED, multiple choice questions, and clinical feedback following each response, was made available online February 23, 2016, and study data were collected through April 11, 2016. An online survey was used to assess the educational effect of the CME program by comparing each participant's responses to 4 identical pre- and post-CME assessment questions. A paired 2-tailed t-test was used to assess whether the mean post-assessment score differed from the mean preassessment score. McNemar's chi-squared statistic was used to determine significance, and effect of education was calculated using Cramer's V, which represents the change in proportion of participants who answered questions correctly from pre- to post-assessment. Cramer's V of $<0.06$ represents no effect, $0.06-0.15$ represents a small change, 0.16-0.30 represents a medium change, and $>0.30$ represents a large change.

RESULTS: Data were collected for 1125 psychiatrists and 785 PCPs who answered all pre- and post-CME assessment questions. Psychiatrists improved significantly in knowledge of diagnostic criteria for BED and 
competence in tailoring evidence-based treatment options ( $\mathrm{P}<.05 ; \mathrm{V}=0.257$; medium educational effect). While only $32 \%$ answered 4 out of 4 questions correctly on pre-assessment, this improved to $84 \%$ on post-CME assessment. PCPs also improved significantly, with $12 \%$ answering 4 out of 4 questions correctly on preassessment, which improved to $70 \%$ on post-assessment $(\mathrm{P}<.05 ; \mathrm{V}=0.266 ;$ medium educational effect $)$.

CONCLUSIONS: Participation in online case-based CME significantly increased understanding of key clinical concepts and competence of physicians treating BED, which has the potential to translate to direct patient benefits. Future education can be designed in case-based formats to further test and educate physicians on diagnosing BED, and increase familiarity and confidence with tailoring evidence-based treatments.

\section{6}

\section{Improving management of major depressive} disorder through virtual patient simulation

Jovana Lubarda, PhD'; Robert Braun'; and Michael E. Thase, $M D^{2}$

\footnotetext{
${ }^{1}$ Medscape Education, New York, NY, USA

${ }^{2}$ Perelman School of Medicine, University of Pennsylvania, Philadelphia, PA, USA
}

ABSTRACT: Study objectives: This study aimed to determine if virtual patient simulation (VPS)-based education could help psychiatrists and primary care physicians (PCPs) improve performance in assessment and management of major depressive disorder (MDD) and adherence to quality improvement measures.

METHODS: Education was delivered online via a unique, interactive, VPS-based learning platform that modeled reallife clinical encounters. Physician learners were presented with two patient cases of MDD, as well as the patients' electronic health records. Following a virtual interaction to obtain patient history, physicians were challenged to order lab tests, make diagnoses, and determine treatment protocols. Possible order options were not limited by multiple choice but rather were supported by a database matching the scope and depth of choices available in actual practice. Clinical decisions made by learners were analyzed using a sophisticated decision engine within the VPS platform, and tailored clinical guidance (CG) was provided in response to each learner decision based on current evidence and expert recommendation. Impact of the education was measured by comparing participant decisions pre- and post-CG using a 2-tailed paired t-test. $\mathrm{P}<.005$ was considered statistically significant.

RESULTS: A total of 878 psychiatrists and 732 PCPs completed both cases. From pre- to post-CG in the simulation, psychiatrists and PCPs were more likely to make evidence-based practice choices in:

- Incorporating measurement-based care, such as evidencebased tools and scales, into patient assessment with an $18 \%$ relative improvement on average for both psychiatrists and PCPs post-CG after completing 2 cases in the simulation. In particular, patient health questionnaire (PHQ-9) utilization increased post-CG for both audiences (from $47 \%$ pre-CG to $67 \%$ post-CG for psychiatrists, $\mathrm{P}=.000$, and from $53 \%$ preCG to $72 \%$ post-CG for PCPs, $\mathrm{P}=.000$ ).

- Individualizing treatment for patients with MDD, including patients who have responded inadequately to initial therapy, by incorporating the latest clinical findings with a $104 \%$ relative improvement on average for both psychiatrists and PCPs in selecting appropriate therapeutic classes post-CG after completing 2 cases in the simulation $(\mathrm{P}<.0005)$, and improvements in nonpharmacological orders such as prescribing cognitive remediation (from $37 \%$ pre-CG to $60 \%$ post-CG for psychiatrists, $\mathrm{P}=.000$, and from $43 \%$ pre-CG to $66 \%$ post-CG for PCPs, $\mathrm{P}=.000$ ).

CONCLUSIONS: The majority of psychiatrist and PCP learners who participated in the VPS-based educational initiative significantly improved their clinical decisions related to utilization of measurement-based care to assess patients with MDD, and selection of evidencebased treatments and nonpharmacologic interventions to improve patient outcomes.

\section{7}

Efficacy of lurasidone in the treatment of major depression with mixed features: the value of early improvement as a predictor of short-term response

Dan V. Iosifesfcu, $M D^{\prime}$; Joyce Tsai, PhD'; Jay Hsu, PhD'; Andrei Pikalov, $\mathrm{MD}, \mathrm{PhD}^{2}$; and Antony Loebel, $M D^{2}$

${ }^{1}$ Icahn School of Medicine at Mount Sinai, New York, NY, USA

${ }^{2}$ Sunovion Pharmaceuticals, Inc, Marlborough, MA, USA, and Fort Lee, NJ, USA

INTRODUCTION: Early identification of patients who are unlikely to eventually achieve a response permits clinicians to intervene early to adjust the dose of medication, or switch to an alternative therapy. Major depressive disorder with mixed features (MDD-MF) has recently been recognized as a diagnostic subtype in DSM-5. No research we are aware of has examined the predictive value of early improvement in patients diagnosed with MDD-MF. The aim of the current post-hoc analysis was to evaluate the value of early improvement in the Montgomery-Åsberg Depression Rating Scale (MADRS) and the Clinical Global Impressions, Severity (CGI-S) scale as predictors of response to lurasidone in patients with MDD-MF. 
METHODS: Patients meeting DSM-IV-TR criteria for MDD, with a MADRS total score $\geq 26$, who presented with 2 or 3 protocol-defined manic symptoms, were randomized to 6 weeks of double-blind treatment with either flexible doses of lurasidone $20-60 \mathrm{mg} / \mathrm{d}$ or placebo. The sensitivity, specificity, positive predictive value (PPV), and negative predictive value (NPV) of early improvement for the prediction of endpoint response (defined as $\geq 50 \%$ reduction from baseline to week 6 in MADRS total score) were estimated for the 6 week completer sample $(\mathrm{N}=189)$. Receiver operating characteristic (ROC) curves were used to evaluate the performance characteristics of early improvement criteria for the prediction of endpoint response, reported as area under the ROC curve (AUCROC). Sustained response at week 3 , 4 , or 5 was defined as meeting response criteria at the respective study week, and continuing to meet response criteria at each subsequent study week.

RESULTS: A significantly higher proportion of patients treated with lurasidone (vs. placebo) met MADRS responder criteria at week $6 \quad(67.6 \%$ vs. $32.2 \%$; $\mathrm{P}<0.001)$. The proportion of patients treated with lurasidone showing sustained response from weeks 3,4 , and 5 through week 6 was $26.5 \%$. $46.1 \%$, and $55.9 \%$, respectively. The proportion of patients treated with lurasidone showing early improvement at Week 2 using the MADRS $\geq 25 \%$ criterion was $46.1 \%$. For prediction of week 6 response, the week 2 MADRS $\geq 25 \%$ improvement criterion had a $56.5 \%$ sensitivity, $75.8 \%$ specificity, $83.0 \%$ PPV, $45.4 \%$ NPV, and AUCROC $=0.70$.

CONCLUSIONS: Week 2 improvement was found to predict clinical response at week 6 in patients treated with lurasidone for MDD-MF. However, the negative predictive value of early improvement was found to be low $(45.4 \%)$ indicating that a majority of patients who did not achieve 25\%-or-greater reduction in their MADRS total score by week 2 nonetheless went on to become responders by week 6 . Further analyses are needed to determine the utility of early improvement for clinical decision-making.

FUNDING: Sponsored by Sunovion Pharmaceuticals Inc. Clinicaltrials.gov identifier: NCT01423240.

\section{8}

Lurasidone adjunctive to lithium or valproate in patients with bipolar I disorder: effectiveness of up to 20 weeks of treatment

Andrei Pikalov, MD, PhD'; Joyce Tsai, PhD'; Yongcai Mao, PhD'; Josephine Cucchiaro, PhD'; and Antony Loebel, $M D^{\prime}$

${ }^{1}$ Sunovion Pharmaceuticals Inc., Fort Lee, NJ, USA, and Marlborough, MA, USA
OBJECTIVE: To evaluate the safety, tolerability, and effectiveness of up to 20 weeks of open label (OL) lurasidone in combination with lithium $(\mathrm{Li})$ or valproate (VPA) for the treatment of bipolar disorder.

METHODS: Patients with bipolar I disorder with $\geq 1$ manic, mixed manic, or depressed episode in the past 2 years, and with a MADRS or YMRS score $\geq 14$ (if on Li or VPA) or $\geq 18$ (if not on Li or VPA) were stabilized during up to 20 weeks of OL treatment with lurasidone $20-80 \mathrm{mg} / \mathrm{d}$ adjunctive with $\mathrm{Li}$ or VPA, and then randomized to 28 weeks of double-blind, placebo-controlled adjunctive lurasidone. We report here on the safety, tolerability, and effectiveness of adjunctive lurasidone during the OL stabilization phase. The effectiveness analyses were limited to non-rapid-cycling patients.

RESULTS: A total of 756 non-rapid-cycling patients (53.4\% with depression; $46.6 \%$ with mania/hypomania/mixed-mania) entered the OL phase, of whom $430(56.9 \%)$ completed up to 20 weeks of open-label treatment, and consented to be randomized in the double-blind phase. For the total safety sample $(\mathrm{N}=962)$, the most common reasons for study discontinuation during open-label treatment were withdrawal of consent $(11.6 \%)$, insufficient clinical response $(11.1 \%)$, lost to follow-up (7.9\%), discontinuation due to adverse events (6.1\%), and miscellaneous other reasons (10.9\%). Among patients with an index episode of depression (baseline MADRS total score $=25.4$; CGI-BP-Sdepression score $=4.2$ ), treatment with adjunctive lurasidone was associated with a mean LOCF-endpoint change in MADRS total score of $\mathbf{- 1 4 . 8}$, and a mean LOCF-endpoint change in CGI-BP-S-depression of -1.9. Responder rates ( $\geq 50 \%$ reduction in MADRS total score) were $70.7 \%$ (LOCF-endpoint) and $85.4 \%$ (completer). Among patients with an index episode of mania/ hypomania/ mixed-mania (baseline YMRS total score $=$ 19.1; CGI-BP-S-mania score=3.7), treatment with adjunctive lurasidone was associated with a mean LOCF-endpoint change in YMRS of -13.6, and a mean LOCF-endpoint change in CGI-BP-S-mania of -1.9 . Week 20 responder rates $(\geq 50 \%$ reduction in YMRS) were $\mathbf{8 2 . 3 \%}$ (LOCF-endpoint) and $\mathbf{9 7 . 3 \%}$ (completer). In the safety sample, the adverse events with an incidence $\geq 5 \%$ were nausea $(11.5 \%)$, headache $(9.1 \%)$, akathisia $(8.3 \%)$, insomnia $(8.0 \%)$, somnolence $(7.2 \%)$, vomiting $(6.1 \%)$, and diarrhea $(5.5 \%)$. Treatment with lurasidone was associated with small median changes $(\mathrm{mg} / \mathrm{dL})$ at LOCF-endpoint in total cholesterol (-2.0), LDL (-3.0), triglycerides $(+12.0)$, glucose $(+1.0)$, and HgbA1c $(0.0)$.

CONCLUSIONS: In patients with bipolar I disorder, up to 20 weeks of treatment with lurasidone, adjunctive with Li or VPA, was associated with clinically significant mean 
improvement in depression and mania scores for patients presenting with index episodes of depression and mania/ hypomania/ mixed-mania, respectively. Treatment with adjunctive lurasidone was safe and well-tolerated.

FUNDING: Sponsored by Sunovion Pharmaceuticals Inc. Clinicaltrials.gov: NCT01358357.

\section{9}

\section{Disseminated cryptococcosis masquerading as psychotic disorder in an immunocompetent host: a case report}

Jungjin Kim, $M D^{\prime}$; Adam Prasanphanich, $P h D^{\prime}$; Lauren Gensler, $M D^{\prime}$; Neguine Rezaii, $M D^{\prime}$; Wendy Baer, $M D^{\prime}$; and Raymond Young, MD, FAPM ${ }^{\prime}$

${ }^{1}$ Emory University School of Medicine, Atlanta, GA, USA

ABSTRACT: Infectious diseases affecting the central nervous system can manifest as a psychiatric illness. When psychiatric symptoms predominate, all too often clinicians are quick to give a psychiatric diagnosis. A rare but possible cause of confusional psychosis is cryptococcal meningitis, which is an opportunistic neuroinfection that can lead to fatality if left untreated. Literature reports of patients with cryptococcal meningitis presenting with confusional psychosis in the absence of constitutional symptoms is sparse, especially in immunocompetent hosts. We report a case of a patient presenting with confusional psychosis that was initially thought to be due to a primary psychotic disorder or a cancer-related organic brain failure, but later was revealed to be due to a disseminated cryptococcal infection.

CASE DESCRIPTION: A 50-year-old woman with no past psychiatric history was transferred to University Hospital with psychosis during workup for lytic bone lesions for presumed malignancy. She complained of a headache but denied fevers or vomiting, and her head CT was normal. Mental status exam revealed poor grooming, significant thought disorder, inappropriately elevated affect, and endorsement of auditory and visual hallucinations. MOCA revealed significant cognitive impairment with prominent attentional and memory deficit. Physical exam was notable only for subcutaneous nodules. Haloperidol was started for presumed delirium or schizophrenia but the patient continued to be psychotic. In addition, the patient developed sudden onset weakness prompting an MRI scan to look for mass lesion, and there were leptomeningeal enhancements and sulcal effacements in the frontotemporal distribution. These findings were consistent with either a brain mass or a herpes simplex virus infection. A lumbar puncture was performed, and the CSF analysis instead revealed cryptococcal antigen. Subsequent workup was positive for cryptococcus in the bloodstream, in the subcutaneous nodule, and in the lytic bone lesions. No malignancy was found and the patient tested negative for HIV. Patient was started on antifungal agents with complete resolution of psychosis achieved at the time of discharge. Initial presentation of this patient was similar to an acute psychotic episode seen in a primary psychotic disorder. Not surprisingly, she was given a provisional diagnosis of schizophrenia in the previous hospital. Early detection of cognitive disturbance led us to correctly identify delirium and direct our attention to searching for the underlying medical cause. Even then, our patient did not show evidence of constitutional symptoms or immune impairment to suspect an infectious etiology. Lytic bone lesions and subcutaneous nodules were more suspicious for malignant processes, which prompted a neoplastic workup. Our chance discovery of cryptococcus as the root cause of psychosis serves as a reminder to clinicians to consider infectious etiologies even in the absence of expected signs and symptoms.

\section{0}

\section{L-methylfolate-induced glycolimia}

Kamran Hanif'; Karam J. Tagore' ${ }^{\prime}$ Nimmi Nirmala', Amisha N. Tailor ${ }^{2}$; Feras A. Shalabi ${ }^{3}$; and Alan R. Hirsch $^{4}$

${ }^{1}$ Caribbean Medical University, Willemstad, Curacao, N.A

${ }^{2}$ Aureus University School of Medicine, Oranjestad, Aruba

${ }^{3}$ St. James School of Medicine, Kralendijk-Bonaire, Netherlands Antilles

${ }^{4}$ Smell and Taste Treatment and Research Foundation, Chicago, IL, USA

ABSTRACT: While sweet craving has been seen with antidepressants, it has never been reported with augmentation with L-methylfolate. Two such cases are described.

METHODS: Case Studies: Case 1: A 57-year-old right handed female presented with burning mouth syndrome, major depression, and generalized anxiety disorder. Despite having undergone treatment with venlafaxine $100 \mathrm{mg}$ BID for one month, she continued to complain of sadness, crying spells, weight loss, impaired appetite, insomnia, low energy level, absent sex drive, easy fatigability, and absent motivation. All her life she has disliked and avoided sweet foods. She was begun on L-methylfolate $15 \mathrm{mg}$ PO qAM. Within 1 week, she noted cravings for sweets and chocolate. After 6 months, the depression resolved, but the glycolimia persisted and she had gained 30 lbs.

RESULTS: Abnormalities on physical examination: General: weight: 122 lbs, height: 66 inches, $1+$ bilateral 
pedal edema. Mental status examination: facial expression: sad and anxious. Poor ability to relax. Oriented x2. Memory Test: Immediate recall: 7 digits forwards, 2 digits backwards. Long term recall: presidents: Obama, Clinton,?. Inability to interpret proverbs. Clock Drawing Test: 4 (normal). Animal Fluency Test: 24 (normal), CNS Lability Scale: 17 (abnormal).

CASE 2: A 52-year-old right handed woman with hyposmia, hypogusia, hypothyroidism, and depression presented with complaints including trouble with memory, anxiety, insomnia, impaired concentration, and absent sex drive. She affirmed worrying most of the time, with frequent panic attacks with tachycardia and overwhelming feelings of anxiety. She presented on citalopram $5 \mathrm{mg}$ PO qAM. Upon treatment with L-methylfolate $5.6 \mathrm{mg}$, methylcobalamin $2 \mathrm{mg}$, and $\mathrm{N}$-acetylcysteine $600 \mathrm{mg}$, she immediately noticed glycolimia with chocolate and sweet food cravings, culminating in a $5 \mathrm{lb}$ weight gain in 3 months.

RESULTS: Abnormalities in Physical Examination: General: weight: $115 \mathrm{lbs}$, height: 64 inches. $1+$ bilateral pedal edema. Mental status examination: Zung Anxiety Scale: Anxiety Index: 41 (normal). Beck Depression Inventory: 6 (normal). Clock Drawing Test: 4 (normal), Semantic Fluency Test: 47 (normal).

DISCUSSION: There are myriad possible mechanisms for L-methylfolate-induced glycolimia. L-methylfolate's function of enhancing the efficacy of the antidepressants may also enhance the side effects of these agents, including sweet cravings and weight gain. Alternatively, L-methylfolate acted to reduce depression, and in response to reducing depression enhanced hedonics and thus facilitated succumbing to hedonic pleasures of sweets, leading to weight gain (Young, 2002). The neurotransmitters norepinephrine, serotonin, and dopamine, which are involved in depression, are also essential for craving (Damsa, 2004). Thus L-methyldopa may be influencing both pathways simultaneously. In those recently started on L-methylfolate, it is worthwhile to monitor cravings, food intake, and weight.

\section{1}

\section{Cerebral amyloid angiopathy related inflammatory process - case report, diagnosis, and treatment}

\section{Kasia G. Rothenberg, MD, PhD'; and Ryan Rajaram, $M D^{\prime}$}

${ }^{1}$ Neurological Institute The Cleveland Cleveland, $\mathrm{OH}$

ABSTRACT: Background: Cerebral amyloid angiopathy related inflammation (CAA-ri), a rare condition caused by an inflammatory reaction occurring within essential cerebral blood vessels against beta-amyloid deposits, leads to subclinical cognitive decline. Often misdiagnosed as dementia, this process can be treated through aggressive immunosuppression, thereby reversing much of the cognitive impairment.

CASE REPORT: We report a 69-year-old female who came to the clinic for a second opinion and had received a previous diagnosis of Alzheimer's dementia $(\mathrm{AD})$ and started on donepezil. She was able to perform ADLs but had difficulties with IADLs. The clinical course was somewhat fluctuating with progressive cognitive symptoms and significant word-finding difficulties. On examination: The patient did exhibit significant wordfinding difficulties and scored $12 / 30$ on MoCA. The patient was also confused and disoriented to time and place. Neurological examination was otherwise unremarkable. Magnetic resonance imaging (MRI) studies were ordered and showed patchy and diffuse T2/FLAIR hyper intensities and particularly concentrated in the posterior cerebral artery and inferior division of the middle cerebral artery. These findings were consistent with cerebral amyloid angiopathy related inflammation (CAA-ri). Besides susceptibility weighted image (SWI) was showing multiple widely distributed microhemorrhages typical for CAA. Imaging showed that the patient's hippocampal volumes were within normal range so this particular imaging biomarker did not support the diagnosis of AD. CSF biomarkers analysis did not support the diagnosis of $\mathrm{AD}$ either since $\mathrm{p}$-Tau levels were found to be within normal limits. Patient was found to be homozygous for the APOE e4 gene. To address the acute inflammatory reaction the patient was hospitalized and received 3 day course of intravenous steroids, followed by an oral steroid taper. Follow-up evaluation (including a repeat MRI study) was performed 2 months later and showed clinical recovery and near complete resolution of diffuse hyperintensities, suggesting inflammation had resolved. Both the patient and the husband reported significant improvement in orientation and other aspects of cognition including working memory. The patient scored 26/30 on MoCA.

DISCUSSION: Cerebral amyloid angiopathy (CAA) has been commonly associated with brain hemorrhages in the elderly, but the inflammatory subtype CAA-I occurs much less frequently and may be often misdiagnosed We present a case of a patient previously diagnosed with $\mathrm{AD}$, upon further investigation, likely CAA-I, treated aggressively with intravenous steroids to good effect. Although rare, CAA-I is a reversible disorder that may be masked by a dementia or/and delirious process and should be considered in patients showing relatively rapid and fluctuating cognitive decline. 


\section{2}

\section{Case report of neuropsychiatric lupus versus steroid-induced psychosis in a young female: mechanisms and management}

Kate Lieb, $M D^{1,2}$; Stephen Percy S. Mondia, $M D^{1,2}$; and Howard L. Forman, MD 1,2

${ }^{1}$ Albert Einstein College of Medicine, Bronx, NY, USA

${ }^{2}$ Montefiore Medical Center, Bronx, NY, USA

ABSTRACT: It is often clinically difficult to distinguish between neuropsychiatric lupus and steroid induced psychosis in lupus patients. Although clinical course, imaging, and laboratory findings can be helpful, they often have limited utility in these complicated cases. This is the case of a 23 yo female with no psychiatric history recently diagnosed with lupus after presenting for syncope with acute blood loss secondary to menorrhagia. Patient was started on prednisone and shortly thereafter developed acute onset of command auditory hallucinations, anxiety, and insomnia. She was taken off prednisone and put on risperidone, at which point psychiatric symptoms remitted for one day but then returned and led to a repeated visit to the psychiatric ER. Patient was noted to be in a lupus flare and required an additional prednisone course, though at a lower dose. While the ideal treatment for steroid induced psychosis involves discontinuing the steroid, this is often not possible in an acute lupus flare. We examine the existing literature on successful treatment of psychosis while continuing steroids. In our case example, we achieved the right combination of prednisone and an antipsychotic; symptoms remitted and the patient was successfully treated.

\section{4}

\section{GeneSight $\circledast$ Psychotropic decreases medication costs for patients with anxiety and bipolar disorders in a large, prospective case-control project}

Ray Lorenz, PharmD'; Kim Horn, MS, LCGC'; Ilia Antonino, PharmD'; Jim Li, MS ${ }^{\prime}$, Lisa Brown, PhD'; and Bryan Dechairo, $P h D^{\prime}$

${ }^{1}$ Assurex Health, Mason, OH, USA

ABSTRACT: Objective: The current objective is to assess the economic utility of the treatment decision support tool, GeneSight Psychotropic, for patients with anxiety and bipolar disorders when healthcare providers (HCPs) make decisions congruent with the GeneSight test.

METHOD: This study is a subgroup analysis of the GeneSight guided group from a larger economic utility study $(\mathrm{n}>13,000)$ that prospectively assessed medication costs in two groups: a GeneSight guided group $(n=1,662)$ and an unguided treatment as usual (TAU) group. Medication costs for 1 year post GeneSight testing were evaluated using pharmacy claims data provided by Medco Research Institute (now Express Scripts). Patients in the GeneSight guided group were divided into two subgroups: congruent (patients whose HCP made treatment decisions congruent with the GeneSight report) and incongruent (patients whose HCP made treatment decisions incongruent with the GeneSight report). This analysis compared total medication cost between congruent and incongruent groups for patients based on their diagnoses of anxiety (GAD), bipolar disorder (BPD), and major depressive disorder (MDD).

RESULTS: Across all evaluated diagnoses, the total savings was $\$ 2774$ (p-value <0.0001) per member per year (PMPY) for patients whose HCP made decisions congruent with the report. Patients with GAD $(n=318$, p-value $<0.0001$ ) saved $\$ 6747$ PMPY when congruent decisions were made, which is greater than the $\$ 3738$ savings in MDD $(\mathrm{n}=459$, $\mathrm{p}$-value $<0.0001)$. In patients with BPD $(n=90)$, congruent decisions saved patients $\$ 4952$ PMPY ( $p$-value $=0.139$ ). Much of the medication cost savings came from antineoplastic, cardiovascular, gastroenterology, diabetes, and CNS medication classes.

CONCLUSIONS: Using GeneSight as a treatment decision support tool for patients with GAD or BPD resulted in significant medication cost savings when HCPs make congruent decisions with the results.

DECLARATION OF FUNDING: This project was fully funded by Assurex Health.

135

It's too salty: hypogeusic hypergeusia

Lily E. Veldran'; and Alan R. Hirsch, $M D^{2}$

${ }^{1}$ Neuroscience Department, Lake Forest College, Lake Forest, IL, USA

${ }^{2}$ Smell and Taste Treatment and Research Foundation, Chicago, IL, USA

ABSTRACT: Hypogeusic hypergeusia (enhanced taste in the presence of true reduced taste) has not heretofore been described.

METHODS: Case study: A 60-year-old female hit the back of her head and lost consciousness. Within a few days, taste was reduced to $20 \%$ of normal, without recovery. After treatment with alpha-lipoic acid, benfotiamine, phosphatidylserine, docosahexaenoic acid, and eicosapentaenoic acid, she developed onset of perceived hypergeusia to salt, whereby salt would taste $150-200 \%$ of normal, which forced her to rinse out her mouth with 
water after eating. When she would add salt to bland food, there was no change in the food's taste.

RESULTS: Chemosensory Testing: Olfactory: Brief Smell Identification: 11 (normosmia). Quick Smell Identification: 2 (hyposmia). Pocket Smell Identification: 2 (hyposmia). Alcohol Smell: 19 (normosmia). Retronasal Smell: Retronasal Smell Index: 2, (reduced). Gustatory: propylthiouricil disc taste: 9 (normoguesia). Taste: normoguesia to sucrose, hydrochloric acid, urea, and phenylthiocarbamide. Mild hypogeusia to salt. Salt water applied to the tongue: salt intensity on the left anterior $3 / 10$, right $0 / 10$, and bilaterally posteriorly 10/10. Artificial sweetener (Splenda) applied to tongue: salt taste pre-application was a $3 / 10$. When sweetener was applied, sweet taste was an $8 / 10$ on the tip of tongue and $5 / 10$ on the middle. Salt taste was not apparent upon application, but returned to a $3 / 10$ intensity 3 minutes post application. Pure sugar applied to tongue: overall salt taste was at a $3 / 10$ intensity. When sugar was applied there was no sweet or salt taste. Three minutes after application the salt taste returned to an intensity of 3/10. Fiberoptic endoscopy negative. CT scan of brain: white matter ischemic changes.

DISCUSSION: A mechanism for this case may be through action of the greater superficial petrosal or glossopharyngeal nerves. These are less receptive than the chorda tympani nerve to $\mathrm{NaCl}$. Maintenance of salt taste in the posterior tongue suggests that the trauma affected the bilateral chorda tympani, with salt projections via pathways in CN IX. In sensorineural hearing loss, low volumes are not detected, while higher volumes are more intense than normal, due to recruitment. Similar recruitment may occur with salt taste. Taste testing for those who present with hypergeusia for presence of hypogeusia is warranted.

\section{6}

\section{Progesterone treatment for phantosmia and phantogeusia}

\author{
Mahipalsinh V. Parmar, $M B B S^{\prime}$; Wannapak Richter, \\ $M D^{2}$; and Alan R. Hirsch, $M D^{3}$ \\ ${ }^{1}$ Rural Medical College, Loni, Maharashtra, India \\ ${ }^{2}$ Ramathibodi Hospital Mahidol University, Bangkok, \\ Thailand \\ ${ }^{3}$ Smell and Taste Treatment and Research Foundation, \\ Chicago, IL, USA
}

ABSTRACT: Introduction: Resolution of phantosmia and phantogeusia with progesterone has not heretofore been described.

METHODS: Case Study: A 25-year-old right handed woman, five years prior to presentation, began to notice foul odors which progressively increased in duration, frequency, and intensity. Six months later she began to notice concurrent taste which also increased in intensity over time. The aroma and taste were fecal and became constant, 7-8/10 in severity. These odors were in both nostrils and worsened with exposure to ambient aromas and exsuflation. The phantom smell and taste overcame any ambient odors or food flavor she consumed. With pregnancies, the phantosmia and phantogeusia resolved and recurred within a few days postpartum. Two days after her most recent delivery, her symptoms recurred, 9-10/10 in severity with constant fecal smell and taste. After beginning daily norethindrone $0.35 \mathrm{mg}$, there has been full resolution of her symptoms for over 3 months.

RESULTS: Chemosensory Testing: Olfaction: Quick Smell Identification Test: 3 (normosmia). Pocket Smell Test: 3 (normosmia). Suprathreshold Amylacetate Odor Intensity Testing: Parallel pattern (normosmia). Sniff Magnitude Test: Sniff Magnitude Ratio: 0.60 (normosmia) Alcohol Sniff Test: $1 \mathrm{~cm}$ (anosmia). University of Pennsylvania Smell Identification Test: Left: 31 (mild hyposmia); Right: 28 (moderate hyposmia). Olfactometer Identification Test: Left: 16.00; Right: 16.00 (hyposmia). Quick Smell Identification Test: 3 (normosmia). Retronasal Olfactory Testing: Retronasal Smell Index: 0 (abnormal). Gustatory Testing: Taste Threshold and Suprathreshold Testing: Normogeusia to sodium chloride, hydrochloric acid and phenylthiocarbamide. Mild hypogeusia (10-30\%) to sucrose and urea.Taste Quadrant Testing: Posterior tongue and palate: weakness to citric acid and quinine hydrochloride. Other Tests: MRI: Left mesial temporal sclerosis. Fiberoptic endoscopy: Normal. Electroencephalogram: Normal

DISCUSSION: Resolution of symptoms during her multiple pregnancies while endogenous progesterone is elevated suggests that this may be the origin of her symptom improvement (Martin, 2006). In rats, progesterone decreases olfactory detection (Phillips, 1975). Possibly progesterone reduces olfactory ability towards the phantom smells and tastes. In this situation the phantogeusia may have represented a disturbance in retronasal smell rather than true taste (Gruss, 2015). On the other hand, progesterone acts on the $\mathrm{t} 2 \mathrm{r}$ receptor which is responsible for bitter taste $(\mathrm{Li}, 2013)$ and in this way may reduce phantogeusia. Alternatively, progesterone may have reduced seizure threshold (Doty, 1986). If the phantoms represented electrical discharges in the insula or uncus, such threshold reduction may have acted to stabilize any electrical discharges, eliminating hallucinations. In those with intractable phantogeusia or phantosmia, a trial on progesterone may be warranted. 
137

\section{Lisdexamfetamine augmentation of hematophobia}

Manisha R. Chand, $M D^{\prime}$; Sumit Kanwar, $M D^{\prime}$; and Alan R. Hirsch, $M D^{2}$

${ }^{1}$ Cleveland Clinic Florida, Weston, FL, USA

${ }^{2}$ Smell and Taste Treatment and Research Foundation, Chicago, IL, USA

OBJECTIVE: Hematophobia afflicts $3 \%$ of the population. Lisdexamfetamine, a prodrug of dextroamphetamine, used in the treatment of attention deficit hyperactivity disorder and binge eating disorder, has been described to exacerbate a plethora of psychiatric disorders. However, exacerbation of phobia, specifically hematophobia, has not heretofore been described.

METHODS: Case Study: A 19-year-old right-handed female presented with complaints of impaired concentration, ease of distractability, lack of focus, and low attention span. It has gradually worsened over the last four years and she has failed two classes in college. She also complained of depression with crying spells, hopelessness, helplessness, and guilt for the last year without suicidal ideation or vegetative symptoms. She affirmed irritability, racing thoughts, and obsessions. She also admitted to a life-long hematophobia whereby she would feel presyncopal with blood draws and thus tries to avoid these or situations involving contact with blood.

RESULTS: Abnormalities in the psychiatric examination include: depressed mood with congruent affect. Mental Status Examination: immediate recall: 7 digits forward, 2 digits backwards. Recent recall: $3 / 4$ objects in 3 minutes and all four with reinforcement. Remote memory: Unable to interpret similarities. Concrete proverbs. Clock drawing test: 4 (normal). Beck Depression Inventory II: 29 (severe depression). Lisdexamfetamine at $20 \mathrm{mg}$ per day induced a marked improvement in her concentration to $70 \%$ of normal, along with a resolution of her anxiety, depression, and obsessions. However, she concurrently noted a marked exacerbation of hematophobia. This was manifest by an increased emotional and physiologic response with syncope on phlebotomy with recurrent blood phobia induced syncope requiring an emergency department visit.

DISCUSSION: Blood-injury-injection phobia is unique in that it causes neurocardiogenic or vasovagal syncope. Those who suffer from it often demonstrate underlying autonomic dysfunction. Lisdexamfetamine increases the release of dopamine and norepinephrine into the synaptic cleft; therefore, with administration of lisdexamfetamine, an enhanced vascular tone would be expected making syncopal episodes less likely.
However, this patient's hematophobia and associated syncope were exacerbated, the mechanism by which is unclear. Lisdexamfetamine potentiates presynaptic release of norepinephrine and dopamine, thus it may have caused a chronic state of sympathetic activation. It is possible that the patient experienced sympathetic activation from the phobic stimulus, allowing unbalanced parasympathetic discharge, and thus syncope. Query and investigation into those with enhanced phobia while taking lisdexamfetamine and other stimulant medications is warranted.

138

\section{Metamorphopsia as a meteorological forecaster}

Mariam Agha'; Anum Wani ${ }^{\prime}$; and Alan Hirsch, MD $^{2}$

${ }^{1}$ Caribbean Medical University School of Des Plaines, IL

${ }^{2}$ Smell and Taste Treatment and Research Foundation, Chicago, IL, USA

ABSTRACT: Introduction: Metamorphopsia, or distortion of shape or continuity of objects (Cytowic, 1989), has not been described to foretell meteorologic changes.

METHODS: Case report: A 51-year-old right handed male, six years prior to presentation, received levofloxacin for a urinary tract infection. Within a short time period he began to experience epochs of "brain fog," characterized by impaired focus, thinking, ability to read, short term memory and disorientation, drowsiness, and anxiety. Along with these were visual changes; shapes became distorted and he could not see the whole picture, only components of it. His visual field became incomplete, as if cut into a jigsaw puzzle, with the pieces are sliding over each other. Also, objects changed; for instance, a portrait of a man was transformed into a picture of a monkey or tiles appeared to be a rug. These occurred one day prior to a storm, were markedly worse hours before the commencement of the storm, and persisted for 3 hours after the storm dissipated. It also occurred on rapid descent on an airplane and resumed a short time after landing and to a lesser degree, on rapid descent in a skyscraper elevator.

RESULTS: Abnormalities in Neurologic Examination: Mental Status Examination: Memory: Immediate Recall: 5 digits forward and 2 backwards. Recent Recall: 2 of 4 objects in 3 minutes and 3 of 4 with reinforcement. Unable to spell "world" backwards. Calculation Ability: Poor. Cranial nerves (CN): CN II: Ophthalmologic exam: Peripapillary pigmentation OU. CN V: Decreased temperature and pinprick in left V1. CN VIII: Hearing intact to CALFRAST, AD 2 strong. Ambassador Hear Pen: AD Absent. IX, X: Gag reflex decreased bilaterally. Uvula deviated to the right. Motor examination: Drift testing: 
right pronator drift. Gait examination: Tandem: Unstable. Cerebellar examination: Slight dysmetria on finger-to-nose bilaterally. Low amplitude high frequency tremor in both upper extremities on extension. Sensory Examination: Romberg: Positive. Reflexes: $3+$ throughout, $4+$ right ankle jerk. Quadriceps femoris pendular bilaterally. Positive Hoffman reflexes bilaterally. Polysomnography: oxygen desaturation to $\mathbf{8 3 \%}$ (obstructive sleep apnea).

DISCUSSION: His visual symptomology suggests metamorphopsia, but also has features of simultanagnosia. Such phenomenon can be seen with migraines (Klee, 1966) or in occipital or parietal lobe seizures (Lee, 2010). The occurrence prior to storms is reminiscent of other hallucinatory phenomenon for instance phantosmia or migraines, which also may appear during such weather changes (Aiello, 2013). Decrease in barometric pressure preceding a thunderstorm may precipitate an amigrainous migraine (Rose, 1986) or may even be a trigger for occipital or parietal lobe seizures (Lee, 2010). Query as to the vaticinating quality of metamorphopsia or simultagnosia is warranted.

\section{9}

\section{Changes in pentylenetetrazole-induced convulsion threshold following chronic caffeine and taurine administration to rats}

\author{
Mariam Sarkhouh'; Mohamed Mubarak'; Mohamed \\ Algherbal'; Ahmed Almahrezi'; Habib Abdulnabi'; \\ Mohammed Naisar ${ }^{\prime}$; and Aamer Kamal ${ }^{\prime}$ \\ ${ }^{1}$ College of Medicine and Medical Sciences, Arabian \\ Gulf University, Manama, Bahrain
}

ABSTRACT: Moderate consumption of caffeine, a xanthine alkaloid, is sufficient to induce behavioral stimulation, which is mainly due to its antagonism of all adenosine receptors that function as neurotransmission and neuronal excitability modulators. In contrast, taurine, a neurotransmitter itself, has a more regulatory effect on the CNS by reducing spontaneous neuronal firing, hyperpolarizing the resting membrane potential and increasing the membrane's conductance for Cl-. The effect of caffeine and taurine on PTZ-induced convulsion parameters was measured following a one-month treatment of caffeine $(0.2 \mathrm{~g} / \mathrm{L}, \mathrm{Caf})$ and taurine $(1000 \mathrm{mg} / \mathrm{kg}$, Taurine) dissolved in the rats' $(\mathrm{n}=136)$ drinking water, as the only source of water available. Furthermore, the probability, latency, and type of convulsions were recorded following the administration of the intraperitoneal PTZ doses. The caffeine treated group showed a significant change in convulsion latency in contrast to the taurine treated group, mainly with the two highest doses of PTZ: $50 \mathrm{mg} / \mathrm{kg}$ (control 376.7 a $63.7 \mathrm{~s}$, Caf $132.4 \mathrm{a} 21.1 \mathrm{~s}, \quad \mathrm{P}<0.05$. Taurine $368.4 \mathrm{a} 88.4 \mathrm{~s}, \quad \mathrm{P}>0.05)$ and $80 \mathrm{mg} / \mathrm{kg}$ (control 281.9 a 63.2s, Caf 127.9 a $44 \mathrm{~s}, \mathrm{P}<0.05$. Taurine $298.4 \mathrm{a} 88.5 \mathrm{~s}, \mathrm{P}>0.05)$. Furthermore, the caffeine treated group also showed a higher probability of convulsion when given the aforementioned doses of PTZ: $50 \mathrm{mg} / \mathrm{kg}$ (control 50\%, Caf 100\%, Taurine 56\%) and $80 \mathrm{mg} / \mathrm{kg}$ (control 67\%, Caf 100\%, Taurine 67\%). Chronic administration of caffeine has a significant effect on the PTZ-induced convulsion threshold. It also shows a higher susceptibility to PTZ than both the control and chronic taurine groups. On the other hand, the chronic administration of taurine seems to have no significant effect on the PTZ-induced convulsion threshold.

140

\section{The incubus strikes again: recurrence of sleep} terrors with lisdexamfetamine administration

Mayur Navinbhai Makwana, MD'; A. N. Tailor ${ }^{2}$; M. L. Walford ${ }^{3}$; K. B. Bartley ${ }^{3}$; and A. R. Hirsch ${ }^{4}$

${ }^{1}$ Pandit Deendayal Upadhyay Medical College, Rajkot, India

${ }^{2}$ Aureus University School of Medicine, Oranjasted, Aruba

${ }^{3}$ Caribbean Medical University, Willemstad, Curaçao

${ }^{4}$ Smell and Taste Treatment and Research Foundation, Chicago, IL, USA

ABSTRACT: Introduction: Use of lisdexamfetamine has been reported to cause multiple sleep disorders, including delayed onset of sleep, insomnia, and nightmares. Night terrors have not heretofore been described to be associated with use of lisdexamfetamine. Such a case is described.

METHODS: Case Study: A 23-year-old male, with a history of childhood night terrors, complained that one month after starting lisdexamfetamine, night terrors resumed. The patient reported that he began having the same terror as he did during his childhood, except now it was recurring three to five times per week. In contrast, as a child, the terror occurred three to four times per year. He recounted the terror as a black figure appearing next to the door or the bed while he was lying in it. The bed would then start to move closer towards the figure. He was unable to speak or to move. He would then scream loudly. His parents responded by waking him up. The patient confirmed one episode of somnambulism as a child, and denied a family history of night terrors or somnambulism. The patient admitted moderate marijuana use in college, but denied recent use of illicit substances or alcohol.

RESULTS: Abnormalities in physical examination: General: Blood Pressure 140/96. Pulse 105. Mental Status Examination: anxious mood with congruent affect. Memory: 
Immediate Recall: able to remember 6 digits forward and 4 digits backwards. Recent recall: able to remember 2 of 4 objects in 3 minutes without improvement with reinforcement. Remote memory: Presidents: Obama, Bush, Clinton, Bush,?. Proverb Testing: concrete interpretation.

DISCUSSION: Night terrors are recurrent episodes of abrupt terror arousals from sleep, usually beginning with a panicky scream, intense fear, and severe autonomic arousal with little to no recollection of dream imagery (DSM-5). The patient's account of the recurrent dream consists of a single, primitive, static image lacking a narrative or kinesthetic component. An atypical feature of his presentation is his ability to recall these events. Perhaps, as an adult, he now has a greater verbal capacity to describe and recall his internal state than he had as a child. The patient's history likely predisposed him to recurrence of the condition as an adult. Possibly, lisdexamphetamine acted to precipitate night terrors by functioning as a dopamine receptor agonist. With increased extracellular concentrations of norepinephrine in the central nervous system as a result of lisdexamphetamine, arousal may occur more easily which then triggered night terrors. Lisdexamfetamine may act to enhance arousal in the reticular activating system so that the patient is more aware of external stimuli in the environment, which functions as a trigger for night terror. Lisdexamfetamine may be inducing night terror indirectly through enhancement of anxiety. After initiating lisdexamfetamine, or another medication in its class, patients should be queried about development of sleep terrors.

141

\section{Pramipexole as a treatment for BMS}

Mayur Navinbhai Makwana, $M D^{\prime}$; and A. R. Hirsch, $M D^{2}$

${ }^{1}$ Pandit Deendayal Upadhyay Medical College, Rajkot, India

${ }^{2}$ Smell and Taste Treatment and Research Foundation, Chicago, IL, USA

ABSTRACT: Introduction: One patient has been described to be responsive to pramipexole for treatment of burning mouth syndrome (BMS) (Stuginski-Barbosa, 2008). Two additional patients are reported.

METHODS: Case 1: A 35-year-old pathological left handed female, three years prior to presentation, when pregnant, developed an unpleasant phantogeusia which resolved on delivery. The following year she began having burning and stinging sensation on the side and tip of the tongue, which has persisted without change. There is diurnal variation in the pain: absent in the morning, progressing to $8 / 10$ in the evening. The pain is better with eating, chewing gum, and moving her tongue. It worsens with drinking coffee and eating dark chocolate. It was unresponsive to plethora of treatments including clonazepam, primidone, vitamins, herbs, and acupuncture. On pramipexole at dose of $1 \mathrm{mg}$ PO QHS, the BMS was markedly better, eliminated most of the time, and only worsening at maximum of $6 / 10$ at the end of the day.

CHEMOSENSORY TESTING: Olfactory Testing: Alcohol Sniff Test (AST): 18 (normosmia). Brief Smell Identification Test (BSIT): 9 (normosmia). Quick Smell Identification Test: 2 (hyposmia). Retronasal Smell Index (RSI): 6 (normal). Gustatory Testing: Propylthiouracil Disc Taste Test: 10 (normogeusia). Taste threshold: Normogeusia to hydrochloric acid and phenylthiocarbamide. Mild hypogeusia (10-30\%) to sucrose and urea. Taste quadrant testing with decreased sucrose anteriorly, decreased citric acid on the left tongue and palate, and decreased alcohol on the posterior tongue, bilaterally.

CASE 2: A 47-year-old right handed woman having previously consulted with $15 \mathrm{MDs}$, several dentists, and an oral surgeon, presented with a four year history of persistent BMS of unknown origin,. Onset of burning was sudden, localized to her tongue, and when severe would spread to the gums, top of the mouth, palate, and even outside her lips. The pain level was 5-8/10 in severity, worst in the evening. It was constant, everyday, and worse with stress, certain food, and strong odors. The BMS was resistant to myriad medications including clonazepam, $\delta$-aminolevulinic acid, diazepam, alprazolam, buspirone, primidone, amantadine, fluconazole, and clonidine. Sleep and tongue movement reduced the pain. After starting pramipexole at dose of $0.25 \mathrm{mg}$ PO QHS, the pain dropped down to an average of 3/10.

CHEMOSENSORY TESTING: Olfactory Testing: AST: 27 (normosmia). BSIT: 11 (normosmia). RSI: 10 (normal). Gustatory Testing: Propylthiouracil Disc Taste Test: 9 (normogeusia). Taste Quadrant Testing: Left palate decreased to sodium chloride. Decreased anterior tongue to all modalities. Quinine hydrochloride impaired throughout. Other: Fungiform Papillae Count: Left 34, Right 32 (normal).

DISCUSSION: These cases confirm and extend StuginskiBarbosa's case study of BMS responsive to pramipexole and suggest a trial of pramipexole for BMS may be worthwhile.

142

\section{A case report of Adderall-induced bizarre delusions}

Michael Harrigan, $\mathrm{MD}^{\prime}$; Rassam Khan, MD; Vandana Doda, MD; and Asghar Hossain, MD

${ }^{1}$ Bergen Regional Medical Paramus, NJ 
ABSTRACT: Introduction: Adderall is a stimulant medication, which is a mixture of $\mathrm{D}$ and $\mathrm{L}$ isomers of amphetamine. Adderall is used in many conditions including ADHD. Chronic and high-dose consumption of Adderall can cause psychosis, presented as delusions, hallucinations, agitation, and anxiety resembling schizophrenia. The purpose of this article is to report the association of Adderall use and psychosis presented as bizarre delusions.

METHOD: Literature search was done using PubMed on "Adderall induced psychosis", "amphetamine overdose", and "amphetamine induced psychosis."

CASE: Our case is a 55-year-old male with a year history of bizarre delusions and visual hallucinations. The patient reported being attacked by gamma rays by airplanes infecting his blood with radiation. These delusions worsened by recent life stressors, first being laid off from his job and second hearing about his family being executed in Syria. The patient was taking prescribed Adderall for $\mathrm{ADHD}$ for last 6 years. He reported taking Adderall more than the prescribed dose. Multiple hospitalizations for delusions were reported in the past year. On examination, he was obese with anxious mood, goal- directed thought-process, and poor insight and judgment. A diagnosis of Adderall-induced psychosis was made and Adderall was discontinued. He was monitored closely for withdrawal symptoms, and a CT scan of head was done which ruled out any intracranial pathobiology. The patient improved within a week of stopping Adderall and was discharged. No psychosis was reported in the follow-up visits at outpatient clinic.

DISCUSSION: Adderall can lead to psychosis with bizarre delusions and hallucination similar to schizophrenia when used chronically and in higher than recommended dose. Physicians should be cautious while prescribing Adderall in patients, as substance abuse is common. More studies are needed to whether life stressors and other social factors (loss of job, execution of family back home in this case) decrease the threshold of development of psychosis in patients taking Adderall.

CONCLUSION: Physicians should screen patients for past history of substance abuse, other psychiatric disorders, and life stressors before prescribing Adderall for various psychiatric disorders.

\section{3}

Efficacy and safety of lurasidone in male and female patients with schizophrenia: a pooled analysis of short-term, placebo-controlled studies

Michael Tocco, PhD'; Diana O. Perkins, $\mathrm{MD}^{2}$; Andrei Pikalov, MD, PhD'; Jay Hsu, PhD'; Josephine Cucchiaro, $\mathrm{PhD}^{\prime}$; and Antony Loebel, $\mathrm{MD}^{\prime}$
${ }^{1}$ Sunovion Pharmaceuticals Inc., Fort Lee, NJ, USA, and Marlborough, MA, USA

${ }^{2}$ University of North Carolina, Chapel Hill, NC, USA

ABSTRACT: Study Objective: To evaluate the efficacy and safety of lurasidone in male and female patients with schizophrenia.

METHOD: Patient-level data were pooled from 5 similarly designed, randomized, double-blind, placebo-controlled, 6-week studies of fixed-dose, once-daily, oral lurasidone $(40,80,120$, or $160 \mathrm{mg} / \mathrm{d})$ conducted in adult patients (age 18-75 years) with acute schizophrenia. Change from baseline to week 6 in Positive and Negative Syndrome Scale (PANSS), Clinical Global Impression-Severity (CGI-S), and Montgomery-Åsberg Depression Rating Scale (MADRS) scores (MADRS data available from 4 of 5 studies) was evaluated using mixed-model repeatedmeasures analysis.

RESULTS: This analysis included 1129 male patients (lurasidone, $\mathrm{N}=757$; placebo, $\mathrm{N}=372$ ) and 416 female patients (lurasidone, $\mathrm{n}=284$; placebo, $\mathrm{n}=132$ ), of whom 1118 male and 414 female patients were included in the intent-to-treat population. Mean PANSS total baseline score was 96.2 for males and 95.9 for females; mean baseline CGI-S score was 4.9 for both groups. Lurasidone effect sizes (Cohen's d) at week 6 in male and female patients, respectively, were 0.53 and 0.59 on the PANSS total score, 0.50 and 0.68 on the PANSS positive subscale, 0.45 and 0.38 on the PANSS negative subscale, 0.45 and 0.50 on the PANSS general psychopathology subscale, and 0.53 and 0.51 on the CGI-S. Mean MADRS score at baseline was 11.6 for males (lurasidone, $\mathrm{n}=682$; placebo, $\mathrm{n}=324$ ) and 12.0 for females (lurasidone, $\mathrm{n}=256$; placebo, $n=124)$. Effect size for change in MADRS score at week 6 was 0.23 in male patients and 0.19 in female patients. The most common adverse events (in $\geq 5 \%$ of lurasidone-treated patients and at least twice the rate observed with patients receiving placebo) for lurasidone versus placebo, respectively, were akathisia $(14.5 \%$ vs $0.8 \%)$, somnolence $(9.4 \%$ vs $4.0 \%)$, and parkinsonism $(5.8 \%$ vs $0.5 \%)$ in male patients, and nausea $(17.3 \%$ vs $3.8 \%)$, somnolence $(12.3 \%$ vs $2.3 \%)$, akathisia $(12.0 \%$ vs $4.5 \%)$, sedation ( $8.1 \%$ vs $0.8 \%)$, parkinsonism $(7.0 \%$ vs $0.0 \%)$, and dizziness $(5.3 \%$ vs $1.5 \%)$ in female patients.

CONCLUSIONS: In this pooled analysis of adult patients with schizophrenia, effect sizes for lurasidone were similar for male and female patients on PANSS total and CGI-S scores. Effect size was somewhat larger on the PANSS positive symptom subscale for female patients compared with male patients. Baseline MADRS scores 
were relatively low, which may have limited the potential for improvement on this measure. Lurasidone was generally well tolerated in both male and female patients with schizophrenia.

FUNDING: Sunovion Pharmaceuticals Inc.

ORIGINAL CITATION: Perkins DO, Tocco M, Pikalov A, Hsu J, Cucchiaro J, Loebel A. Efficacy and safety of lurasidone in male and female patients with schizophrenia: a pooled analysis of short-term, placebo-controlled studies. Accepted for presentation at the 29th ECNP Congress; September 17-20, 2016; Vienna, Austria.

\section{4}

\section{A case report and literature review of clozapine re-challenge titration schedules}

Mina Boazak, MD, PGY-1'; Lindsay Cox, BS, MS4', James Ragazino, MD, PGY-1'; Michael Lowley, MD, $P G Y-1^{\prime}$; Hassan Naqvi, MD, PGY-1'; Danielle Epstein, $B A, M S 4^{3}$; and Robert Cotes, $M D^{\prime}$

${ }^{1}$ Emory University, Atlanta, GA, USA

${ }^{2}$ Philadelphia College of Osteopathic Medicine, Philadelphia, PA, USA

${ }^{3}$ Nova South Eastern College of Osteopathic Medicine, Lauderdale, FL, USA

STUDY OBJECTIVE: In light of recent findings suggesting a relationship between clozapine-induced myocarditis and clozapine titration rates, we attempt to assess whether a similar relationship exists between clozapine and its hematological side effects (neutropenia/agranulocytosis). In this report we discuss this topic as it relates to clozapine rechallenge, by presenting a case of a rechallenged patient as well as our findings from a literature review.

METHODS: Patient case history was obtained from chart review. Our literature review was conducted on PubMed under the search headings "clozapine rechallenge" and "clozapine after neutropenia". Article titles and abstracts were assessed and eliminated when not meeting criteria. Remaining articles were assessed for data relating to titration rate and white blood cell (WBC)/ absolute neutrophil count (ANC) values.

RESULTS: We present a case of a 49 y/o Caucasian female with hx of schizoaffective disorder who successfully responded to a clozapine rechallenge after previous discontinuation for an episode of moderate neutropenia while on a dose of $600 \mathrm{mg}$ daily. Rechallenge was conducted over a period of roughly 5 months to a current dose of $300 \mathrm{mg}$ of clozapine. Adjunctive olanzapine $(40 \mathrm{mg})$ was used for symptoms and lithium $(900 \mathrm{mg})$ for its leukocytotic effects. Analysis of the patient's clozapine dose and serum levels against WBC/ANC levels via Pearson's correlation was unrevealing. We also discuss the absence of data in our literature review, with only 4 articles discussing titration schedules without ambiguity.

CONCLUSION: There exists a paucity of literature discussing the impact of clozapine titration schedules on WBC/ ANC levels in clozapine rechallenges. Further studies will need to be conducted to shed light on this important patient safety matter. In the interim we suggest adhering to current guidelines for titration rates.

145

Mania-like symptoms induced by dopamine agonist treatment in a patient with hyperprolactinemia: a case report and a review of the literature

Mohamed Abd Elalem Aziz, MD'; Ahmed Negida ${ }^{2}$ Mohamed Soliman, $\mathrm{MD}^{3}$; and Sally Ghali, $\mathrm{MD}^{3}$

${ }^{1}$ Air Force Specialized Hospital, Cairo, Egypt

${ }^{2}$ Zagazig University, Zagazig, Egypt

${ }^{3}$ University of California at Los Angeles, Los Angeles, CA, USA

ABSTRACT: Subject Objective: Cabergoline is an oral, dopamine receptor agonist used for the treatment of hyperprolactinemia, Parkinson's disease, and uterine fibroids. Cabergoline is recognized by its long half-life (65 hours), and its effect can continue for 21 days after usage. Common adverse effects of cabergoline include nausea, constipation, and psychiatric symptoms like depression and somnolence. However, to the best of our knowledge, this is the 2 nd case report in the literature to describe mania like symptoms with dopamine agonist. Hereby, we report a case of cabergoline-induced mania with psychotic features in a patient with hyperprolactinemia.

METHOD: A 22-year-old, newly married female, diagnosed with infertility due to hyperprolactinemia, was presented to the psychiatric unit four days after her first dose of cabergoline (Dostinex). Her family described changes in behavior, insomnia, self-talking, euphoria, and false claims. On psychiatric evaluation, the patient appeared euphoric and loudly-laughing with psychomotor hyperactivity, grandiose behavior, flight of ideas, and delusions of persecutions. Her mood was labile with impaired abstract, judgment, and pressured speech. Young Mania Rating Scale was 40. MRI brain and laboratory tests showed no abnormalities. The patient was diagnosed with mania with psychotic features.

RESULTS: Cabergoline treatment was immediately stopped and the case was successfully managed with 
atypical antipsychotics (aripiprazole) and mood stabilizer (carbamazepine). After three weeks, the patient showed greater improvement and all psychiatric symptoms disappeared.

CONCLUSION: In this case, stopping dopamine treatment, and giving atypical antipsychotics and mood stabilizer treated the mania-like episodes. The prescription of dopamine agonists should be avoided in susceptible individuals.

FUNDING: This research received no specific grant from any funding agency in the public, commercial, or not-forprofit sectors.

\section{6}

\section{Acyclovir-induced nephropathy in an encephalitis patient: a case report and review of literature}

Mohamed Abd Elalem Aziz, MD'; Ahmed Negida ${ }^{2}$, Mohamed Elsayed Helmy, MD'; Sherien Mohamed Farag, $\mathrm{MD}, \mathrm{PhD}{ }^{\prime}$; Mohamed Soliman, $\mathrm{MD}^{3}$; and Sally Ghali, $M D^{3}$

${ }^{1}$ Air Force Specialized Hospital, Cairo, Egypt

${ }^{2}$ School of Medicine, Liverpool University, Liverpool, UK

${ }^{3}$ University of California at Los Angeles, Los Angeles, CA, USA

ABSTRACT: Subject Objective: Acyclovir is an antiviral drug used in herpes and varicella infections causing encephalitis. Serious adverse events in patients treated with acyclovir are rare. Hereby, we report a case of an Egyptian patient, treated with acyclovir for viral encephalitis, who developed nephropathy.

METHOD: A 35-year-old male was admitted to the hospital with fever refractory to antipyretic medications. Although the patient was drowsy and inattentive, with progressive decline in conscious level, the MRI brain revealed no signs of encephalitis. However, laboratory results showed: (1) CSF analysis: an increase in CSF proteins and CSF cells to 400 cells $/ \mathrm{mm} 3$, (2) CBC: increase in total leucocyte count to 17.41 thousand/ cmm, (3) CSF culture showed no evidence of bacterial infection, and (4) normal renal functions tests. A diagnosis of viral encephalitis was reached. Acyclovir intravenous injection $750 \mathrm{mg} / 8$ hours was given and the patient kept under monitoring. After 3 days on acyclovir treatment, renal function tests increased suddenly (creatinine level $6.9 \mathrm{mg} / \mathrm{dl}$ ) with progressive deterioration in the conscious level.

RESULTS: Renal function tests improved gradually with adjustment of intravenous acyclovir to $750 \mathrm{mg} / 24$ hours and the patient's consciousness returned to normal.
CONCLUSION: The present case showed that high-dose intravenous acyclovir might induce nephropathy due to direct renal tubular toxicity, acute interstitial nephritis, and crystal nephropathy. High dose intravenous acyclovir should be prescribed with caution for patients with viral encephalitis.

FUNDING: This research received no specific grant from any funding agency in the public, commercial, or not-forprofit sectors.

\section{7}

\section{Crossed hemiparesis is the main presentation in aortic dissection case: a case report and review of literature}

Mohamed Abd Elalem Aziz, MD'; Ahmed Negida'; Tarik Ibrahim Menecie, MD, PhD'; Mohamed Soliman, $M D^{3}$; and Sally Ghali, $M D^{3}$

${ }^{1}$ Air Force Specialized Hospital, Cairo, Egypt

${ }^{2}$ School of Medicine, Liverpool University, Liverpool, UK

${ }^{3}$ University of California at Los Angeles, Los Angeles, CA, USA

ABSTRACT: Subject Objective: Stroke secondary to aortic dissection (AD) usually causes rapid deterioration and death. Clinical suspicion is essential for detection and prevention of serious complications. Herby, we report a case of stroke secondary to $\mathrm{AD}$, which was correctly managed.

METHOD: An Egyptian 33-year-old hypertensive male was admitted to the emergency department with crossed hemiparesis and dysarthria associated with feeling of tightness in his chest for four hours. ABC algorithms for cardiac and stroke patients were done to stabilize the patient. Laboratory tests and brain CT were normal. ECG revealed no ischemic changes but with prolongation of QTc interval. When measuring the blood pressure in both arms, the pulse in the right arm was not palpable. Computerized tomographic angiography (CTA) of chest revealed presence of Type A aortic dissection.

RESULTS: Following surgical repair, the patient showed an improvement with gradual disappearance of neurological manifestations. Blood pressure was controlled and the patient was discharged after three weeks.

CONCLUSION: Chest pain is not always the main presentation of $\mathrm{AD}$. A few $\mathrm{AD}$ cases can present with neurological manifestation. Rapid surgical repair operation is important to prevent the serious complications.

FUNDING: This research received no specific grant from any funding agency in the public, commercial, or not-for-profit sectors. 
148

Schizophrenia discovered accidently during an ENT examination of a patient: a case report and review of literature

Mohamed Abd Elalem Aziz, MD'; Ahmed Negida ${ }^{2}$; Mohamed Soliman, $\mathrm{MD}^{3}$; and Sally Ghali, $\mathrm{MD}^{3}$

${ }^{1}$ Air Force Specialized Hospital, Cairo, Egypt

${ }^{2}$ School of Medicine, Liverpool University, Liverpool, UK

${ }^{3}$ University of California at Los Angeles, Los Angeles, CA, USA

ABSTRACT: Subject Objective: The ability of medical staff to suspect psychiatric symptoms of their patients is important for rapid referral and proper management of the underlying psychiatric disorder.

METHOD: A 22-year-old male was admitted with dizziness and discharge from his left ear. During the ENT examination, the patient claimed that the magnetic resonance imaging instrument (MRI) is broadcasting his thoughts to be controlled by the doctor (thought broadcasting and control). The patient was referred to the psychiatric unit and the mental status examination showed positive symptoms of schizophrenia: delusions of persecution, auditory hallucinations, Thought disorders and impaired judgment. MRI showed no organic cause of the psychiatric symptoms and therefore, the patient was diagnosed with schizophrenia and received the proper management.

RESULTS: This patient was managed with anti-psychotic medications (risperidone) and mood stabilizer (carbamazepine). The patient was followed until his condition improved after one month, then he was discharged from hospital to continue cognitive behavioral therapy (CBT) in outpatient clinic.

CONCLUSION: The training of medical staff, other than psychiatrists, to suspect psychiatric symptoms in their patients is important for early detection and preventing deterioration of undiagnosed or missed psychiatric patients.

FUNDING: This research received no specific grant from any funding agency in the public, commercial, or not-forprofit sectors.

\section{9}

\section{Mania-like symptoms in a patient with traumatic} brain injury: a case report and review of literature

Mohamed Abd Elalem Aziz, MD'; Ahmed Negida ${ }^{2}$; Mohamed Soliman, $\mathrm{MD}^{3}$; and Sally Ghali, $\mathrm{MD}^{3}$

${ }^{1}$ Air Force Specialized Hospital, Cairo, Egypt

${ }^{2}$ School of Medicine, Liverpool University, Liverpool, UK

${ }^{3}$ University of California at Los Angeles, Los Angeles, CA, USA
ABSTRACT: Subject Objective: Traumatic brain injury (TBI) causes a broad spectrum of neurological and psychiatric symptoms. Mania-like symptoms are not common with TBI. Hereby, we report a case of mania-like symptoms induced by hyponatremia in a patient with TBI.

METHOD: A 43-year-old Egyptian male with a history of TBI in the previous month and no history of psychiatric disorders. The patient showed a sudden, progressive change in behavior including pressured speech, impulsivity, elevated mood, inflated self-esteem (grandiosity), and insomnia for the last four days. Mini Mental State Examination of the patient showed: (1) an increase in rate and amount of speech and psychomotor activity, (2) intact short-term memory, (3) delusions of grandeur, (4) good judgment, and (4) no obvious deficit in abstract thinking. Lab investigations showed a progressive decline in serum sodium level with decrease of urine volume and an increase in antidiuretic hormone $(\mathrm{ADH})$ level. The patient was diagnosed with the syndrome of inappropriate antidiuretic hormone (SIADH) secretion.

RESULTS: The patient improved after correction of sodium level with $500 \mathrm{ml}$ of $3 \%$ intravenous $\mathrm{NaCl}$ for 48 hours. Patient's mood returned gradually to normal with no impulsivity and disappearance of grandiose delusions, and the patient was discharged from the hospital after four weeks.

CONCLUSION: Recurrent mania-like episodes, caused by electrolyte disturbances such as hyponatremia due to traumatic brain injury, can be reversed with proper management of hyponatremia condition and underlying organic cause.

FUNDING: This research received no specific grant from any funding agency in the public, commercial, or not-forprofit sectors.

\section{0}

Gene delivery of AAV2-neurturin for patients with Parkinson's disease: a meta-analysis

Ahmed Elmaraezy'; Hussien Ahmed'; Mohamed Elnenny'; Ahmed Negida ${ }^{2}$; Mohamed Abd Elalem Aziz, $\mathrm{MD}^{3}$; Mohamed Soliman, $\mathrm{MD}^{4}$; and Sally Ghali, $\mathrm{MD}^{4}$

${ }^{1} \mathrm{Al}$ Azhar University, Cairo, Egypt

${ }^{2}$ Zagazig University, Zagazig, El-Sharkia, Egypt

${ }^{3}$ Air Force Specialized Hospital, Cairo, Egypt

${ }^{4}$ University of California at Los Angeles, Los Angeles, CA, USA

BACKGROUND: There is unmet need for developing better treatment strategies for Parkinson's disease (PD). 
Gene delivery of neurturin, a natural analogue for glial derived neurotrophic factor, showed promising results in animal models and early open label trials. However, its efficacy has not been confirmed yet.

OBJECTIVE: The objectives of this meta-analysis are: 1) to investigate the efficacy of AAV2-neurturin gene delivery for patients with Parkinson's disease; 2) to compare $\mathrm{AAV} 2$-neurturin delivery in putamen only versus in putamen plus substantia nigra; and 3) to compare efficacy of gene delivery before 18 months and after 18 months of the injection.

METHODS: We searched PubMed for clinical trials (single arm and controlled trials) assessing the efficacy of AAV2-neurturin gene delivery in patients with PD. Data were extracted from eligible studies and were analyzed using RevMan and open meta[analyst] statistical packages. We conducted meta-regression to investigate whether the improvement in unified Parkinson's disease rating scale (UPDRS III motor score) was associated with the target of gene delivery or follow-up duration.

RESULTS: Four studies ( $\mathrm{n}=123$ patients) were pooled in the final analysis. Compared with sham surgery, neurturin gene delivery was not significantly superior before 18 months but after 18-24 months (UPDRS III: MD $-0.39,95 \%$ CI [ -4.15 to 3.36 ] vs. MD $4.49,95 \%$ CI [ -8.63 to -0.35$]$, respectively). Intraputamenal gene delivery was significantly better than gene delivery in putamen plus substantia nigra (UPDRS III: MD -12.76 , 95\% CI [15.61 to 9.92$]$ vs. MD $-6.72,95 \%$ CI $[-10.38$ to -3.06]; $\mathrm{P}=0.01$ ).

CONCLUSIONS: Current evidence is insufficient to confirm the efficacy of AAV2-neurturin gene delivery. Our results suggest that the efficacy of AAV2-neurturin gene delivery in the putamen only should be evaluated by further randomized controlled trials with follow-up duration beyond 18 months.

FUNDING: This research received no specific grant from any funding agency in the public, commercial, or not-forprofit sectors.

\section{1}

\section{Bapineuzumab for Alzheimer's disease: a systematic review and meta-analysis}

Abdelrahman Ibrahim Abushouk, MD'; Ahmed Elmaraezy ${ }^{1,2}$; Samar Fouda ${ }^{2,3,4}$; Amro Aglan ${ }^{2,5}$; Reham Salama ${ }^{2,5}$; Mohamed Abd Elalem Aziz, MD ${ }^{6}$; Mohamed Soliman, $\mathrm{MD}^{7}$; and Sally Ghali, $\mathrm{MD}^{7}$

${ }^{1}$ Faculty of Medicine, Al Azhar University, Cairo, Egypt
${ }^{2}$ NOVA-med Research Association, Cairo, Egypt

${ }^{3}$ Faculty of Medicine, Zagazig University, Zagazig, Egypt

${ }^{4}$ Student Research Unit, Zagazig University, Zagazig, Egypt

${ }^{5}$ Faculty of Medicine, Benha University, Benha, Egypt

${ }^{6}$ Air Force Specialized Hospital, Cairo, Egypt

${ }^{7}$ University of California at Los Angeles, Los Angeles, CA, USA

ABSTRACT: Introduction: Alzheimer's disease (AD) is a globally prevalent neurodegenerative condition, clinically characterized by progressive memory loss and gradual cognitive functions impairment. Passive immunotherapy with monoclonal antibodies such as bapineuzumab has been proven to reduce amyloid $\beta$ protein load in the brain in preclinical and human trials. We performed a systematic review of published randomized clinical trials (RCTs) to investigate the safety and efficacy of bapineuzumab in patients with mild to moderate $\mathrm{AD}$.

METHODS: A computer literature search of PubMed, OVID, EBSCO, Scopus, EMBASE, Web of Science, and Cochrane CENTRAL has been conducted using relevant keywords. Eligible studies were included after abstract and full text screening. Heterogeneity was measured by chi-square and I-square tests. Statistical analysis was performed by Review Manager Version 5.3 for windows.

RESULTS: Six RCTs $(n=2380)$ were eligible for the final analysis. The overall effect estimate did not favor bapineuzumab over placebo regarding the clinical outcomes including; ADAS-Cog11 $(\mathrm{MD}=0.14,95 \% \mathrm{CI}$ $[-0.72,0.99]), \mathrm{DAD}(\mathrm{MD}=1.35,95 \%$ CI $[-1.74,4.43])$, CDR-SOB $(\mathrm{MD}=0.21,95 \% \mathrm{CI}[-0.07,0.49]$, and MMSE $(\mathrm{MD}=0.08,95 \% \mathrm{CI}[-0.31,0.47])$. Analysis of the key biomarker outcomes showed that bapineuzumab significantly reduced the concentration of phosphorylated tau proteins (SMD = -5.53, 95\% CI [-8.29, -2.76]). However, bapineuzumab was not superior to placebo in terms of SUVR (SMD $=-0.56,95 \%$ CI $[-1.24,0.13])$ and MRI whole-brain volume measurement $(\mathrm{SMD}=0.09,95 \% \mathrm{CI}$ $[-0.02,0.21])$. In terms of safety, bapineuzumab increased the risk of serious TEAEs $(\mathrm{RR}=1.18,95 \%$ CI [1.02-1.37]) and amyloid related imaging abnormalities $(\mathrm{RR}=40.88,95 \%$ CI $[11.94,135.95])$. None of the other adverse events were higher in the bapineuzumab group compared to placebo group.

CONCLUSION: Although bapineuzumab effectively reduced phosphorylated tau protein CSF concentration, this was not associated with improved clinical outcomes. Moreover, bapineuzumab significantly increased the risk of serious TEAEs and amyloid related imaging abnormalities. In light of the current evidence, bapineuzumab 
should not be used for treatment of patients with mild to moderate $\mathrm{AD}$.

152

\section{Safinamide as add-on therapy for Parkinson's} disease: a meta-analysis of randomized controlled trials

Hussien Ahmed, MD'; Hussien Ahmed ${ }^{1,2,3}$; Attia Attia $^{1,4}$; Shimaa Elkholy ${ }^{1,5}$; Omnya Osama ${ }^{1,5}$; Mohamed Abd Elalem Aziz",6; Zainab Hassan ${ }^{1,2,3}$; Ahmed Elmaraezy ${ }^{1,4,7}$; Ahmed Negida ${ }^{1,2,3}$; Mohamed Soliman, $M D^{8}$; and Sally Ghali, $M D^{8}$

${ }^{1}$ Medical Research Group of Egypt, Cairo, Egypt

${ }^{2}$ Faculty of Medicine, Zagazig University, Zagazig,

Elsharkia, Egypt

${ }^{3}$ Student Research Unit, Zagazig University, Zagazig,

El-Sharkia, Egypt

${ }^{4}$ Faculty of Medicine, Al Azhar University, Cairo, Egypt

${ }^{5}$ Kasr Al ainy school of Medicine, Cairo University, Cairo, Egypt

${ }^{6}$ Air Force Specialized Hospital, Cairo, Egypt

${ }^{7}$ NOVA-med Research Association, Cairo, Egypt

${ }^{8}$ University of California at Los Angeles, Los Angeles, CA, USA

ABSTRACT: Objectives: The objective of this meta-analysis is to synthesize evidence from published randomized controlled trials about the efficacy of safinamide as addon therapy for patients with Parkinson's disease.

INTRODUCTION: Patients with Parkinson's disease (PD) on chronic levodopa treatment suffer from motor fluctuations and levodopa induced dyskinesia. Investigators are searching for add-on therapies to improve patients' response and reduce treatment induced complications. Safenamide is an aminoamide derivative with a dual mechanism of action including both dopaminergic and non-dopaminergic circuits. Safinamide has been under evaluation, in multiple clinical trials, as add-on therapy for patients with PD.

METHODS: We searched PubMed for randomized controlled trials (RCTs) assessing the efficacy of safinamide as add-on therapy for patients with PD. Eligible studies were selected and data were extracted and analyzed using open meta[analyst]. Changes in Unified Parkinson's Disease Rating Scale (UPDRS) part III and part II and Clinical Global Impression Scale (CGI-S) were pooled as mean difference between the two groups (safinamide versus placebo) from baseline to endpoint in a metaanalysis model.

RESULTS: Four RCTs ( $\mathrm{n}=1432$ patients) were pooled in the final analysis. The overall effect size favored safinamide group in terms of UPDRS III (MD -1.82, 95\% CI [ -2.59 to -1.06$]$ ), UPDRS II (MD $-1.55,95 \%$ CI [ -2.23 to -0.88$]$ ), and CGI-S (MD $-0.38,95 \%$ CI [ -0.49 to -0.28$]$ ). Data about the reduction in levodopa dose and "off" time could not be pooled because they were not adequately reported in published studies.

CONCLUSION: Our results show that safinamide slightly improved clinical symptoms of PD. However, current evidence is inadequate to confirm the efficacy of safinamide as add-on therapy for PD. We recommend further randomized controlled trials to evaluate the efficacy of safinamide in reducing levodopa dose and "off" time.

\section{3}

\section{Prevalence of brain imaging findings in patients} on an inpatient psychiatric unit

Mohammad Mashayekh, MD'; Ali Khadivi, PhD; and Panagiota Korenis, $M D$

${ }^{1}$ Bronx Lebanon Bronx, NY

ABSTRACT: Advances in neuroimaging have revolutionized medicine and aided in our understanding of how biological abnormalities may contribute to clinical presentation. Brain imaging, in more advanced variants, is implicated in primary and secondary prevention of psychiatric disorders. While such advances have also begun to enhance our knowledge about the timing of developmental deviations, it remains unclear at this time how neuroimaging impacts the clinical course of the patient. Currently, there are contradictory publications and guidelines for the application of conventional brain imaging (MRI and CT) in the evaluation of patients with psychosis.

METHOD: We conducted a pilot retrospective chart review of patients who were admitted to the inpatient psychiatric service over a period of 12 months starting July 1 , 2015 through June 30, 2016. Patients in the study were selected randomly among patients ages 18 years and older, who were admitted to the inpatient service, and had brain imaging.

RESULTS: We aim to review the incidence of brain structural abnormalities and present the prevalence and common imaging findings in a sample of our patient population.

CONCLUSIONS: While imaging advances have added to our understanding of biological abnormalities and can aid in ruling out organic causes of psychiatric illness, imaging does not guide our current management of patients. 
154

\section{Repeated transient ischemic attacks induced by sexual intercourse: case report and review of literature}

Mohamed Abd Elalem Aziz, MD'; Mohammed Elsayed Helmy, $\mathrm{MD}^{\prime}$; Mohamed Soliman, $\mathrm{MD}^{2}$; and Sally Ghali, $M D^{2}$

${ }^{1}$ Air Force Specialized Hospital, Cairo, Egypt

${ }^{2}$ University of California at Los Angeles, Los Angeles, CA, USA

ABSTRACT: Subject objective: Sexual intercourse is considered a rare precipitating factor of ischemic stroke in risk group patients. We present a case of female patient with repeated transient ischemic attacks (TIA) during sexual intercourse.

METHODS: A 24-year-old right handed female on oral contraceptive presented with repeated attacks of acute onset vertigo, right homonymous hemianopia, right lower limb weakness, and dysarthria that occurred during sexual intercourse. These symptoms completely resolved after $10 \mathrm{~min}$. MRI brain with diffusion showed decreased diffusion in left occipital lobe, MR angiogram evidenced a vasospasm in posterior cerebral artery (PCA), and the autoimmune profile was negative.

RESULTS: Patient management was with antiplatelet drug (clopidegrol) $75 \mathrm{mg} /$ day. Follow up for one year showed no repetition of the attacks.

CONCLUSION: Ischemic cerebral events, such as TIA or ischemic stroke, have been reported to be caused during sexual intercourse. Several mechanisms explaining this brain insult, such as vasospasm of cerebral arteries during intercourse, have been discussed but none of them was totally proved. Combined physical, radiological, and laboratory examination may be useful in prevention of recurrence of TIA.

FUNDING: This research received no specific grant from any funding agency in the public, commercial, or not-forprofit sectors.

155

Anxiety to confusion: CYP interaction to SIADH

Pankaj Lamba, $M D^{\prime}$

${ }^{1}$ Behavioral Medicine and Psychiatry, West Virginia University School of Medicine, Morgantown, WV, USA

ABSTRACT: Drug-drug interactions through GYP-450 enzymes and drug-induced syndrome of inappropriate secretion of antidiuretic hormone (SIADH) is often discussed at length in medical textbooks. However, diagnosis is not that straightforward unless physicians are vigilant. Older patients and certain medications, such as SSRIs and omeprazole, have been implicated in development of SIADH. The writer presents one case to highlight the issue.

CASE REPORT: A 67-yr-old Caucasian man started experiencing anxiety in March. Stressor was his dying girlfriend. His past psychiatric history was unremarkable. Medical history was significant for HTN, hyperlipidemia, BHP. His PCP, on $3 / 2 / 16$, advised citalopram $10 \mathrm{mg}$ and omeprazole $20 \mathrm{mg}$ daily for anxiety and GERD. He was taking hydrochlorothiazide and lisinopril. He visited ED several times, in March, with complaints of anxiety and abdominal pain. Work-up did not uncover any organic etiology and alprazolam PRN was advised for anxiety. His girlfriend passed away in April and he presented to ED several times in May with complaints of anxiety, abdominal pain, insomnia, and racing heart. On 5/15, citalopram was titrated to $15 \mathrm{mg}$ daily and omeprazole to $20 \mathrm{mg}$ BID. The client was seen by the writer on $6 / 14$. He appeared anxious and complained of mental fog, lethargic feeling, loss of appetite, and dizziness. A review of labs showed a gradual decline of serum sodium levels; on $3 / 12$ - 139 then on $5 / 10$ - 138, 5/22 -128, $5 / 31-125$. He was advised a taper of citalopram to $5 \mathrm{mg}$, decrease by $5 \mathrm{mg} \mathrm{q} 3$ days. A repeat sodium on 6/23 was 133 . He reported an improvement, felt calmer with clear mentation. A diagnosis of medication-induced SIADH along with adjustment $\mathrm{d} / \mathrm{o}$ was considered.

DISCUSSION: The patient was on several medications that may cause SIADH, namely hydrochlorothiazide, lisinopril, citalopram, and omeprazole. He demonstrates hyponatremia due to SIADH after titration of dosage of citalopram and omeprazole. On tapering citalopram, the $\mathrm{Na}$ level trended towards normalization. Both citalopram and omeprazole are metabolized through CYP2C19, and omeprazole has been implicated in decreasing hepatic metabolism of citalopram.

CONCLUSION: A review of literature was performed. Cases of hyponatremia in patients taking both omeprazole and citalopram/escitalopram have been reported. In most of the cases the patient's sodium level was closer to the lower limit of normal and they were older (known risk factor for developing hyponatremia) and experienced rapid decline in sodium level, within a week. Caution is therefore advised when treating older patients with omeprazole and citalopram/ escitalopram concomitantly. If they present with sudden or unexplained mental status changes (e.g., delirium, lethargy, confusion) or nausea, hyponatremia due to drug-induced SIADH should be on differential. 
156

Medication-assisted treatment and

pharmacogenetics to facilitate sustained engagement for c0-occurring disorders

Patricia Allen, DNP, PMHNP-BC $C^{\prime}$; and Elizabeth Drew, MD, FASAM ${ }^{2}$

${ }^{1}$ Summit Behavioral Health, Nursing Cincinnati, OH

${ }^{2}$ Summit Behavioral Health, Medical Cincinnati, OH

ABSTRACT: Heroin addiction is an epidemic impacting millions of Americans. Deaths from this dangerous drug have increased by $286 \%$ from 2002 to 2013 . Additionally more than $65 \%$ of those with a substance use disorder also have a co-occurring mental health disorder. Successful addiction treatment must be multidisciplinary and encompass both the mental health and substance use disorders. Medication-assisted treatment and pharmacogenetics testing are essential tools that support engagement and recovery. This Doctor of Nursing Practice (DNP) project explored engagement outcomes for clients with an opiate use disorder receiving treatment in two outpatient programs. Outcomes were evaluated at 30, 60 , and 90 days to determine the efficacy of medication assisted treatment and pharmacogenetics testing in those with a co-occurring disorder. A retrospective chart analysis was conducted on 158 clients engaged in treatment between April 1, 2015 and March 31, 2016. Demographic, diagnostic, and pharmacogenetics data were collected for 158 clients with an opiate addiction. Eighty of the 158 received either suboxone or Vivitrol. Findings indicated that $65 \%$ remained engaged in treatment at 90 days compared to $37 \%$ of those without medication-assisted treatment. Of the 158 clients, 92 were diagnosed with a co-occurring disorder. Eighty-one had pharmacogenetics testing. At 90 days $90 \%$ of those with a co-occurring disorder remained actively engaged in treatment.

\section{7}

Effects of aripiprazole once-monthly and paliperidone palmitate on work readiness in patients from the QUALIFY study stratified by age

Phyllis Salzman, PhD'; Steven G. Potkin, $M D^{2}$; Jean-Yves Loze, MD, MSc ; Carlos Forray, $\mathrm{MD}^{4}$; Ross A. Baker, PhD, MBA'; Christophe Sapin, MSc'; Timothy Peters-Strickland, $\mathrm{MD}^{\prime}$; Maud Beillat, $\mathrm{MSc}^{5}$; Anna-Greta Nylander, PhD, MBA ${ }^{6}$; Peter Hertel, $\mathrm{PhD}^{6}$; Simon Nitschky Schmidt, MSc ; Anna Eramo, $\mathrm{MD}^{7}$; Karina Hansen, $P h D, M S c^{5}$; and Dieter Naber, $M D^{8}$

${ }^{1}$ Otsuka Pharmaceutical Development \& Commercialization, Inc., Princeton, NJ, USA

${ }^{2}$ Department of Psychiatry and Human Behavior, University of California, Irvine, CA, USA
${ }^{3}$ Otsuka Pharmaceutical Europe, Wexham, United

Kingdom

${ }^{4}$ Lundbeck LLC, Paramus, NJ, USA

${ }^{5}$ Lundbeck SAS, Issy-les-Moulineaux, France

${ }^{6}$ H. Lundbeck A/S, Valby, Denmark

${ }^{7}$ Lundbeck LLC, Deerfield, IL, USA

${ }^{8}$ Department for Psychiatry and Psychotherapy, University Medical Center Hamburg-Eppendorf, Hamburg, Germany

ABSTRACT: Background: The QUALIFY (QUAlity of LIfe with AbiliFY Maintena) study compared treatment effectiveness of aripiprazole once-monthly $400 \mathrm{mg}$ (AOM 400) to paliperidone palmitate once-monthly (PP) showing superior improvements with AOM 400 vs PP on health-related quality of life and functioning (Naber et al., 2015). As an additional outcome in QUALIFY, patients' functional capacity to work was assessed with the clinician-rated Work Readiness Questionnaire (WoRQ, Potkin et al., 2016). Increasing work readiness could be of particular importance in younger patients with schizophrenia, and here we present age-stratified analysis on capacity to work and work readiness.

METHODS: QUALIFY was a 28-week, randomized, openlabel, head-to-head study (NCT01795547) of 2 atypical long-acting injectable anti-psychotics, AOM 400 and PP (flexible dosing per label) in patients with schizophrenia. Included patients were age 18-60 years needing a change from current oral antipsychotic treatment. The protocol pre-specified effectiveness analyses in patients $\leq 35$ years and $>35$ years and recruitment targeted $30 \%$ of patients $\leq 35$ years. WoRQ was rated at baseline and at week 28/ end-of-study, and total scores range from 7-28 (lower scores indicate better functioning). Lastly, the clinician records if the patient is ready for work (Yes/No). Analysis of covariance was applied to changes from baseline in WoRQ total scores, and logistic regression was used to estimate odds ratios for work readiness at week 28 , adjusted for work readiness status at baseline.

RESULTS: At baseline, WoRQ total scores and frequencies of work readiness were similar between AOM 400 and PP treatment groups in patients $\leq 35$ years $(\mathrm{n}=54)$ and $>35$ years $(\mathrm{n}=154)$. In patients $\leq 35$ years, significantly greater improvements with AOM 400 vs PP were found in WoRQ total scores at week 28 (least squares mean treatment difference: -2.70 ; 95\%CI: [-4.41; -0.99$]$, $\mathrm{p}=0.0026)$. Similarly, shifts from No to Yes in work readiness were more frequent after AOM $400(38 \%$ [12/32]) vs (9\% [2/22]) PP treatment, and the odds of being rated as ready for work at week 28 were significantly better for AOM 400 vs PP treatment (adjusted odds ratio: 14.7; 95\%CI: [2.48, 87.2], $\mathrm{p}=0.0031$ ). In patients $>35$ years, numerically larger 
improvements were seen after AOM 400 vs PP treatment on WoRQ total score and on work readiness at week 28 .

CONCLUSIONS: Significantly greater improvements on WoRQ total scores and work readiness in patients $\leq 35$ years from QUALIFY support increased functional capacity after AOM 400 vs PP treatment. Numeric improvements with $\mathrm{AOM} 400$ vs $\mathrm{PP}$ were observed in patients $>35$ years. These results indicate that increased capacity to work and work readiness are attainable treatment goals in schizophrenia, and may be of particular importance in younger patients.

\section{REFERENCES:}

Naber D, et al. Schizophr Res. 2015; 168: 498-504.

Potkin SG, et al. CNS Spectr. 2016; 21: 199-206.

\section{8}

Identification of prodromal signs of dementia: anxiety and insomnia in the aging, demographics, and memory study

\section{Richard Wallis, MSN, PMHNP, PhD}

${ }^{1}$ Student at the University of Texas Medical Branch at Galveston, TX, Psychiatric Nurse Practitioner at Denver Denver, CO

ABSTRACT: Study Objective: The objective of this study is to evaluate the relationship between anxiety and insomnia (A/I) symptoms and subsequent dementia in the Aging, Demographics, and Memory Study (ADAMS).

METHODS: The ADAMS is a supplement to the Health and Retirement Study (HRS). Wave A took place from 20012003 and included extensive neuropsychological evaluation. The final follow-up assessment battery took place from 2008-2009 (Wave D). For the present analysis, we will evaluate the relationship between A/I symptoms in Wave $\mathrm{A}$ and presence of dementia in Wave D.

RESULTS: Wave A included evaluation of 856 participants over the age of 71 . For Wave D, 217 participants underwent final evaluation. Drop-off in the first 3 waves was due to the identification of dementia $(n=393)$, death $(n=151)$, or unspecified reasons $(n=165)$. This poster will present preliminary dissertation findings.

CONCLUSIONS: Identification of prodromal indicators of developing dementia may lead to earlier diagnosis, treatment, and the development of novel strategies to prevent further cognitive decline.

FUNDING: The ADAMS study is a supplement to the Health and Retirement Study (HRS), which is sponsored by the National Institute of Aging (grant number NIA U01AG009740). It was conducted jointly by Duke
University and the University of Michigan. No funding or financial support was received for this presentation.

159

\section{Incarceration of the mentally ill}

Richard Wallis, MSN, PMHNP, PhD'; and Andreas Brueggler, MSN, PMHNP

${ }^{1}$ Student at the University of Texas Medical Branch at Galveston, TX, Psychiatric Nurse Practitioner at Denver Health

${ }^{2}$ Psychiatric Nurse Practitioner at Denver Denver, CO

ABSTRACT: Study Objectives: The objectives of this presentation are to discuss the historical development of incarceration of the mentally ill and to evaluate assisted outpatient treatment (AOT) as a potential solution to preventing incarceration for mental illness.

METHOD: The establishment of state hospitals as a way to divert the mentally ill from jails and prisons will be explained. We will describe the progression and context of the movement toward de-institutionalization that was boosted by the development of psychiatric medications and the idea that community mental health could manage the mentally ill outside of hospitals. We will then describe the social and economic costs of transinstitutionalization from healthcare institutions to jails and prisons. Finally, AOT models will be presented as a potential solution to managing the mentally ill.

RESULTS: Incarceration of the mentally ill is a costly and inhumane way to manage mental illness. AOT is one potential model for managing individuals who are otherwise incarcerated as a result of their untreated mental illness.

CONCLUSIONS: Assisted outpatient treatment is a humane and cost-effective model for diverting the mentally ill from jails and promoting independence.

FUNDING: No funding or financial support was received for this study.

160

The efficacy and safety of lurasidone in adolescent patients with schizophrenia: a 6-week, double-blind, placebo-controlled, multicenter study

Robert S. Goldman, PhD'; Robert L. Findling, MD, $M B A^{2}$; Robert Silva, PhD'; Josephine Cucchiaro, $P h D^{\prime}$; Ling Deng, PhD'; and Antony Loebel, $M D^{\prime}$

${ }^{1}$ Sunovion Pharmaceuticals Inc., Fort Lee, NJ, USA

${ }^{2}$ Johns Hopkins University and the Kennedy Krieger Institute, Baltimore, MD, USA 
ABSTRACT: Study Objective: To evaluate the efficacy and safety of lurasidone in adolescent patients with schizophrenia.

METHOD: Adolescents (13-17 years old) diagnosed with schizophrenia (DSM-IV-TR criteria; Positive and Negative Syndrome Scale [PANSS] total score?70 and Clinical Global Impression-Severity (CGI-S) score?4) were randomly assigned to 6 weeks of double-blind treatment with fixed-dose lurasidone $40 \mathrm{mg} / \mathrm{d}, 80 \mathrm{mg} / \mathrm{d}$, or placebo. Changes from baseline to week 6 in the primary (PANSS total score) and key secondary (CGI-S) efficacy outcome measures and in body weight were evaluated using mixed-model repeated-measures analysis.

RESULTS: A total of 326 patients (mean age $=15.4$ years) received lurasidone $40 \mathrm{mg} / \mathrm{d} \quad(\mathrm{N}=108), \quad 80 \mathrm{mg} / \mathrm{d}$ $(\mathrm{N}=106)$, or placebo $(\mathrm{N}=112)$. The PANSS total score at week 6 demonstrated a placebo-adjusted, least-squares (LS) mean improvement of -8.0 $(\mathrm{p}<0.001$, effect size $[E S]=0.51)$ for the $40 \mathrm{mg} / \mathrm{d}$ group and $-7.7(\mathrm{p}<0.001$, $\mathrm{ES}=0.48$ ) for the $80 \mathrm{mg} / \mathrm{d}$ group. On the CGI-S, the placebo-adjusted, LS mean improvement at week 6 was $-0.47(\mathrm{p}<0.001, \mathrm{ES}=0.49)$ for the $40 \mathrm{mg} / \mathrm{d}$ group and $-0.42(p=0.002, E S=0.45)$ for the $80 \mathrm{mg} / \mathrm{d}$ group. The rate of study discontinuation due to adverse events (AEs) was higher in placebo- versus lurasidone-treated patients (8.0\% vs 3.7\%). The most common AEs (incidence?5\% in either lurasidone group and? twice the rate of placebo) for lurasidone $40 \mathrm{mg} / \mathrm{d}, 80 \mathrm{mg} / \mathrm{d}$, and placebo, respectively, were nausea $(12.7 \%, 14.4 \%, 2.7 \%)$, somnolence $(9.1 \%$, $11.5 \%, 5.4 \%)$, akathisia $(9.1 \%, 8.7 \%, 1.8 \%)$, vomiting $(8.2 \%, 6.7 \%, 1.8 \%)$, and sedation $(5.5 \%, 1.9 \%, 1.8 \%)$. LS mean change in weight at week 6 for the lurasidone $40 \mathrm{mg} / \mathrm{d}, 80 \mathrm{mg} / \mathrm{d}$, and placebo groups was $0.17 \mathrm{~kg}$, $0.49 \mathrm{~kg}$, and $0.05 \mathrm{~kg}$, respectively. Median change at week 6 end point in total cholesterol was $-4.0 \mathrm{mg} / \mathrm{dL},-2.0 \mathrm{mg} /$ $\mathrm{dL}$, and $-7.0 \mathrm{mg} / \mathrm{dL}$, respectively; in triglycerides was $-2.0 \mathrm{mg} / \mathrm{dL}, 7.0 \mathrm{mg} / \mathrm{dL}$, and $-2.0 \mathrm{mg} / \mathrm{dL}$, respectively; and in glucose was $0.0 \mathrm{mg} / \mathrm{dL}, 1.0 \mathrm{mg} / \mathrm{dL}$, and $0.0 \mathrm{mg} / \mathrm{dL}$, respectively. Median change in prolactin level at week 6 (LOCF) in males was $0.8 \mathrm{ng} / \mathrm{mL}, 1.0 \mathrm{ng} / \mathrm{mL}$, and $0.0 \mathrm{ng} /$ $\mathrm{mL}$ for the lurasidone $40 \mathrm{mg} / \mathrm{d}, 80 \mathrm{mg} / \mathrm{d}$, and placebo groups, respectively; and in females was $0.6 \mathrm{ng} / \mathrm{mL}$, $4.4 \mathrm{ng} / \mathrm{mL}$, and $0.7 \mathrm{ng} / \mathrm{mL}$, respectively.

CONCLUSIONS: In this study, lurasidone $(40 \mathrm{mg} / \mathrm{d}$ and $80 \mathrm{mg} / \mathrm{d}$ ) demonstrated statistically significant efficacy and clinically meaningful symptom improvement in adolescent patients with schizophrenia. Lurasidone was generally well tolerated, and was not associated with clinically meaningful effects on body weight, lipids, glucose, or prolactin.

FUNDING: Sunovion Pharmaceuticals Inc.
ORIGINAL CITATION: Goldman R, Findling RL, Silva R, Cucchiaro J, Deng L, Loebel A. The efficacy and safety of lurasidone in adolescent patients with schizophrenia: a 6-week, double-blind, placebo-controlled, multicenter study. Submitted for presentation at the 63rd Annual Meeting of the American Academy of Child \& Adolescent Psychiatry; October 24-29, 2016; New York, NY.

\section{1}

\section{Clozapine-induced myocarditis: a case report and literature review}

\section{Sahil Munjal, $M D^{\prime}$}

${ }^{1}$ PGY-4 (Chief Resident), New York Medical College at Westchester Medical Valhalla, NY

ABSTRACT: Clozapine is an atypical antipsychotic with a well-known efficacy for the treatment of resistant psychosis. Clinicians are well aware of the drug's metabolic and hematologic side effects. Less attention is given to clozapine's cardiac side effects such as myocarditis, despite a high associated mortality rate. The need to consider myocarditis as one of the causes of flu-like symptoms in patients taking clozapine should be well emphasized as early intervention might improve the prognosis of the patient significantly. We are presenting a case report of a 37-year-old male with schizoaffective disorder who developed myocarditis within 3 weeks of starting on clozapine for his treatment resistant psychosis. Once clozapine-related myocarditis has been diagnosed, it is mandatory to immediately stop clozapine treatment. Early cessation of clozapine treatment may improve the clinical outcomes. Considering the high mortality, there is a proposed monitoring protocol. Cessation of clozapine is advised if troponin is more than twice the upper limit of normal or C-reactive protein is over $100 \mathrm{mg} / \mathrm{L}$. Combining these two parameters has shown an estimated sensitivity for symptomatic clozapine-induced myocarditis of almost $100 \%$.

\section{2}

\section{Ciprofloxacin-induced exacerbation of anxiety in an elderly patient: a review of anxiogenic potential of fluoroquinolones}

Sahil Munjal, $M D^{\prime}$; and Yvette Smolin, $M D^{2}$

${ }^{1}$ PGY-4, New York Medical College at Westchester Medical Center

${ }^{2}$ Liaison Service, New York Medical College at Westchester Medical Valhalla, NY

ABSTRACT: Fluoroquinolones are one of the most commonly prescribed antibiotics worldwide and in general, they are well tolerated. Yet, fluoroquinolones, including 
ciprofloxacin, can cause neuropsychiatric side effects including insomnia, anxiety, and depression, which may go unnoticed. A recent analysis of the side effects of ciprofloxacin in the U.S. Food and Drug Administration (FDA) Adverse Event Reporting System (AERS) database showed that of all the side effects reported on the medication, $10 \%$ experienced anxiety. The anxiogenic effects could be related to inhibition of the GABA receptor complex and activation of NMDA receptors as evidenced in the rat studies. Reviewing the literature, there is not a single case reported of an elderly patient who developed exacerbation of anxiety due to ciprofloxacin. In light of the absence of literature, we present a case of an 85-year-old female with a history of generalized anxiety disorder who was stable at baseline and presented with an exacerbation of anxiety post her treatment of a UTI with ciprofloxacin. We review the literature on the anxiogenic potential of ciprofloxacin and fluoroquinolones, in general.

\section{3}

\section{Metamorphoses of phantosmia in temporal lobe epilepsy — a case study}

\author{
Samreen Siddiqui ${ }^{\prime}$; and Alan R. Hirsch, MD, FACP ${ }^{2}$ \\ ${ }^{1}$ Ziauddin Medical College, Ziauddin University, \\ Karachi, Pakistan \\ ${ }^{2}$ Smell and Taste Treatment and Research Foundation, \\ Chicago, IL, USA
}

ABSTRACT: Introduction: While phantosmia is seen in $5.5 \%$ of those with temporal lobe epilepsy (Chen, 2003), fluctuation in the nature of this hallucination in the same individual has heretofore been reported.

METHODS: Case study: An 81-year-old, right handed woman developed an upper respiratory tract infection followed by daily attacks of knife-like sensation beginning behind the left ear and moving to the left eye. This was followed by left hemicephalgia, which radiated to the left neck, instantly followed by a feeling of mouth dryness and grittiness. As this comes on, the headache subsides. Within seconds after this, she experiences shortness of breath and anxiety which lasts for less than 5 minutes, ending with a sudden onset (8/10 in intensity) phantosmia, lasting 15 seconds coinciding with a burning sensation in her nose. It was consistent from epoch to epoch, but over months, transformed in quality from the aroma of burning wires to the smell of paint and then to cigarette smell and finally to natural gas. She admits to epochs of forceful thinking, speech arrest with dyslexia, non-fluent speech where she has words in her mind but cannot speak them, comprehension deficits where she is unable to understand questions, anomia, and déj土 vu. She also experiences 'dream-like states' with auditory and visual hallucinations consisting of shadows in the right peripheral visual field lasting a few seconds, every few months, for the last four years.

RESULTS: Abnormalities in neurological examination: Mental status: recent recall: 0/4 objects in three minutes and 2/4 with reinforcement. Clock Drawing Test: 3 (abnormal). Animal Fluency Test: 16 (abnormal). Unable to interpret similarities. Motor drift: right cerebellar spooning. Gait: heel walking with decreased right arm swing. Sensory: decreased light touch and pin prick in all four extremities. Reflexes: 0-1+ throughout. Other: Olfactory testing: Brief Smell Identification test: 12 (normosmia). Alcohol Sniff Test: 22 (normosmia). Retronasal Smell Index: 4 (abnormal). Gustatory: Propylthiouracil Disc Taste Test: 10 (normogeusia). MRI of brain with and without infusion: periventricular and subcortical deep white matter chronic ischemic changes. Electroencephalogram: Polymorphic slowing with theta waves in the left temporal lobe

DISCUSSION: This may indicate a pathophysiological fluctuation in the primary focus of the seizure. This could be contextually related, with the poorly differentiated phantosmia misattributed to environmental cues. With temporal lobe seizures, there is an increase in ipsilateral nasal engorgement (Hirsch, 1999). This would facilitate air flow of ambient aromas to the olfactory epithelium and thus enhanced olfactory ability. Further investigations in the natural course of phantosmia are warranted.

\section{4}

\section{Genetic polymorphisms in treatment-resistant schizophrenia and response to clozapine}

\author{
Ethan Isidero, $\mathrm{MD}^{3}$ \\ ${ }^{1}$ Bergen Regional Medical Center, NJ, USA \\ ${ }^{2}$ Research Volunteer \\ ${ }^{3}$ Medical Student IV
}

Sarah Sheikh, MD'; Lara Adesso, MD'; and

ABSTRACT: Introduction: Studies have shown that approximately one-fifth to one-third of patients with schizophrenia do not respond to first and second-line treatment. Treatment-resistant schizophrenia (TRS) is defined by the International Psychopharmacological Algorithm Project as a failure to respond to at least two 4-6 week trials of monotherapy with either typical or atypical antipsychotics. Clinical trials have shown significantly greater improvements in TRS patients who are on clozapine compared to typical antipsychotics such as chlorpromazine. However, due to the hazardous sideeffect profile of clozapine, it is not commonly considered as a first-line agent for schizophrenia. Certain studies suggest that there may be an association between TRS 
and specific genetic polymorphisms, particularly on the genes that code for dopamine and serotonin receptors. The discovery of a distinct genetic association with TRS would greatly benefit treatment, as patients could potentially be screened early for TRS and would no longer be required to undergo up to 12 weeks of sub-optimal treatment.

EDUCATIONAL OBJECTIVE: In this review, the authors review numerous genetic studies regarding polymorphisms that may be associated with either TRS or an increased response to clozapine. The authors also explore the genetic and social phenotype of TRS patients and the potential use of genetics in the workup of schizophrenia.

CASE: We report a case of a 40 -year-old Caucasian male with a psychiatric history of schizoaffective disorder bipolar type presenting with worsening of psychotic symptoms including constructional, grandiose, persecutory, and somatic delusions. He has an extensive history of treatment-resistance to antipsychotic medications including Zyprexa and Risperdal, but has displayed improvement of symptoms on clozapine during hospitalization.

CONCLUSION: Evidence shows that there is clearly a genetic component to TRS, both in risk of development and response to clozapine. However, more research must be done in order to warrant both genetic screening in schizophrenic patients and approval of clozapine as a first-line treatment.

\section{5}

\section{Takotsubo syndrome induced by antipsychotic medication along with other psychiatric treatments: literature review}

Sarah Sheikh, $M D^{\prime}$; Annie Al-Najjar, $M D^{2}$; and Adel Neshiewat ${ }^{3}$

\footnotetext{
${ }^{1}$ Bergen Regional Medical Center, NJ

${ }^{2}$ Research Volunteer

${ }^{3}$ Medical Student 4th Year
}

ABSTRACT: Introduction: Takotsubo syndrome, also known as "apical ballooning syndrome," is a cardiomyopathy induced by stress. It manifests clinically as transitory dysfunction of the left ventricle mimicking symptoms of acute myocardial infarction. Takotsubo syndrome is an important syndrome that needs to be well understood by psychiatrists. Its pathology is not completely known but there are reported studies on the association of Takotsubo syndrome with antipsychotic medications, as well as lithium and ECT.

OBJECTIVE: Our goal is to study the cause-and-effect relationship of antipsychotic medication in addition to other psychiatric treatments with the development of Takotsubo syndrome.
METHODS: A literature search via PubMed and Google scholar has been conducted on Takotsubo xyndrome and its relation to antipsychotic medication and other psychiatric treatment.

DISCUSSION: A number of studies have been performed to establish the relation of Takotsubo syndrome and antipsychotic medication and other antipsychotic treatment modalities. In one study, the authors hypothesized that the Takotsubo syndrome may have been triggered by an exacerbation of psychiatric illness, which features high baseline levels of catecholamines that could have been further elevated by the stress experienced during the suicide attempt. In another study, a young man developed Takotsubo syndrome in the context of clozapine initiation and maintenance ECT; he improved after discontinuation of clozapine. In a case report of an elderly woman with bipolar disorder, it was reported that she developed Takotsubo syndrome in the context of lithium toxicity. The use of antipsychotics, specifically olanzapine and amisulpride, and the mood-stabilizer lithium was associated with prolonged QT interval and risk of torsades de pointes. When the dose was lowered, there was improvement. However, when the medications were used again, there was reappearance of life-threatening ventricular arrhythmia. Anxiodepressive disorder and chronic psychological stress could participate in the pathophysiology of the development of Takotsubo syndrome. It was found that there was a tendency for patients with Takotsubo syndrome to use more psychiatric medications (anxiolytic, antidepressants, or antipsychotic drugs). In a different study, it is hypothesized that clozapine causes cardiotoxicity; however the mechanism is unclear. An elderly woman in a different study with a history of antidepressant, antipsychotic medication developed Takotsubo syndrome after ECT, suggesting that ECT can be an iatrogenic trigger for Takotsubo syndrome. Clozapine causes higher noradrenaline levels; hence, increased plasma catecholamine levels have recently been implicated in Takotsubo cardiomyopathy. There are several studies that have reported higher incidence of Takotsubo syndrome in middle aged women. Therefore, further more specific epidemiological studies are warranted.

166

The effects of valbenazine (NBI-98854) on tardive dyskinesia: results of a randomized, double-blind, placebo-controlled, phase 3 trial (KINECT 3)

Stephen R. Marder, $M D^{\prime}$; Robert A. Hauser, $M D^{2}$; Scott Siegert, PharmD ${ }^{3}$; Clinton Wright, PharmD ${ }^{3}$; and Christopher F. O'Brien, $M D^{3}$

${ }^{1}$ Semel Institute for Neuroscience at UCLA 
${ }^{2}$ University of South Florida Parkinson's Disease \& Movement Disorder Center

${ }^{3}$ Neurocrine Biosciences, San Diego, CA

ABSTRACT: Objectives: Tardive dyskinesia (TD) is a persistent and often disabling movement disorder associated with chronic exposure to dopamine receptor blocking agents, such as antipsychotics. There are currently no treatments FDA-approved for TD. However, valbenazine (VBZ; NBI-98854) is a novel and highly selective vesicular monoamine transporter 2 (VMAT2) inhibitor that is in development for the treatment of TD. Following Phase 1 and 2 trials that demonstrated favorable efficacy and tolerability profiles, VBZ was evaluated in a Phase 3 trial (KINECT 3; NCT02274558).

METHODS: KINECT 3 was a 6-week, double-blind, parallel-group, placebo (PBO)-controlled trial in subjects with moderate-to-severe TD and underlying schizophrenia, schizoaffective disorder, or mood disorder. Subjects were randomized $(1: 1: 1)$ to once-daily treatment with PBO, VBZ $40 \mathrm{mg}$, or VBZ $80 \mathrm{mg}$. The primary outcome was an intent-to-treat (ITT) analysis of change from baseline at Week 6 on the Abnormal Involuntary Movement Scale (AIMS) for VBZ $80 \mathrm{mg}$ vs PBO, assessed by blinded central video raters. Safety assessments included adverse event (AE) rates, laboratory tests, electrocardiograms (ECG), and psychiatric assessments, including the Positive and Negative Syndrome Scale (PANSS), Calgary Depression Scale for Schizophrenia (CDSS), Young Mania Rating Scale (YMRS), Montgomery-Åsberg Depression Rating Scale (MADRS), and the Columbia Suicide Severity Rating Scale (C-SSRS).

RESULTS: In the safety population $(\mathrm{n}=227), 150(66 \%)$ subjects had schizophrenia/schizoaffective disorder and 194 (85\%) were receiving $\geq 1$ concomitant antipsychotic medication ( $16 \%$ typical, $77 \%$ atypical). In the ITT population $(\mathrm{n}=225)$, the mean baseline AIMS score (SD) was 10.1 (4.0). VBZ $80 \mathrm{mg}$ vs PBO resulted in a significant improvement from baseline at Week 6 in AIMS score: least squares (LS) mean change, -3.2 vs $-0.1 ; \mathrm{P}<0.0001$. The AIMS score was also reduced with VBZ $40 \mathrm{mg}$ vs PBO: LS mean change, 1.9 vs -0.1 ; $\mathrm{P}=0.0021$ (full description of supportive analyses to be presented). AE rates were similar across all groups and were consistent with prior studies. The most commonly reported $\mathrm{AE}$ across both $\mathrm{VBZ}$ groups was somnolence: VBZ $80 \mathrm{mg}$, 5.1\%; VBZ $40 \mathrm{mg}$, 5.6\%; PBO, 3.9\%. Six percent of all subjects discontinued due to treatmentemergent AEs: VBZ $80 \mathrm{mg}, 6.3 \%$; VBZ $40 \mathrm{mg}, 5.6 \%$; PBO, 5.3\%. Mean score changes on the PANSS, CDSS, YMRS, MADRS, and C-SSRS were similar between VBZ and PBO.
CONCLUSIONS: Once-daily VBZ was associated with a significant improvement in TD. Both doses of VBZ appeared to be well tolerated in subjects with schizophrenia/schizoaffective disorder or mood disorder, even when taken with a wide range of concomitant medications, including typical and atypical antipsychotics. Psychiatric scales suggested no increased risk in psychiatric symptoms, depression or suicidality with VBZ during the trial.

167

Neuropsychiatric manifestation of 5HTR2C gene deletion in a child: first reported case in the world

Shajüddin Faraz Mohammed, PGY $2^{\prime}$; and Alexander Lerman, $M D^{\prime}$

${ }^{1}$ New York Medical College/Westchester Medical Center, Valhalla, NY, USA

ABSTRACT: HPI: Pt is a 6 yo AAM with past history of seizure disorder presented to Psych ER for evaluation after displaying aggressive and homicidal behavior at home. As per mother, patient was behaving normally at his therapist's office the same day, but after returning home, he started acting out and became aggressive when limits were set. He pulled out a kitchen knife and tried to hurt his mother. She was able to grab it and sent him to his room. Patient went to his room, hid in a closet for some time and came out angry and tried to attack his grandmother with a knife. Mother contacted the therapist and patient was brought to Psych ER for evaluation. No c/o depression, mania, anxiety, nightmares, or flashbacks. No h/o physical or sexual abuse. No recent change in neurovegetative symptoms like recent change in sleep/appetite/energy or concentration. Pt had a past history of paranoia, visual hallucinations, aggression, unsteady gait, and head banging behavior.

PPH: Pt was admitted twice to psych hospital in the past for self-injurious behavior. Pt was first admitted to a psych hospital at the age of 4 for biting, screaming, kicking, aggression, hitting head against the wall, and trying to cut himself. Pt also c/o paranoia, visual hallucinations, and unsteady gait periodically. Patient was initially on escitalopram/aripiprazole and risperidone but was later switched to escitalopram/quetiapine and VPA. Visual hallucinations were well controlled on VPA.

DIAGNOSIS: 24 hour Video EEG was done. It was consistent with diffuse or multifocal cerebral dysfunction. Target events of agitation, visual hallucinations were captured. Patient was agitated and combative at times and during these periods, seizures were captured. With the introduction of valproic acid, there was a marked improvement both in the EEG and the patient's clinical behavior. He was diagnosed with 5HTR2C gene mutation after chromosomal 
analysis and DNA sequencing. He was the first reported case of 5HTR2C gene deletion in the world.

\section{8}

Treatment of unipolar, nonpsychotic major depressive disorder with transcranial magnetic stimulation: examining acute and long-term efficacy of TMS

Kimberly Cress, $M D^{\prime}$; Sherry Grogan, APRN, PMHNP$B C^{\prime}$; Kerstin Brown, BA'; Beth Landry'; and Roxane Zotyka'

${ }^{1}$ TMS Serenity Sugar Land, TX

ABSTRACT: Background: Major depressive disorder (MDD) affects approximately 16 million lives in the U.S. (6.7\% of adults) with about $50 \%$ seeking help and only $20 \%$ receiving adequate treatment. Transcranial magnetic stimulation (TMS) is noninvasive, non-systemic therapy that uses pulsed magnetic fields to induce localized neuronal depolarization and beneficial effects on the symptoms of MDD. The purpose of this review is to evaluate standardized symptom score outcomes in routine clinical practice and establish the long-term efficacy of TMS.

METHODS: 120 patients with a primary diagnosis of unipolar MDD who had not received benefit from antidepressant treatment (average of 3.7 in current MDD episode) received TMS treatment. Each patient was assessed using the Beck Depression Inventory scale. Scores were performed prior to and at the end of the acute treatment phase. Long-term results were reported on those patients that returned for assessment.

RESULTS: The study population included average age 48.4 years with $69.2 \%$ female. The mean TMS sessions were 41.1 with a range of 1,600 to 4,600 pulses. 94 patients (78.3\%) demonstrated a minimum $50 \%$ improvement in the BDI-II symptom score while 84 patients $(70.0 \%)$ achieved remission with reported symptom scores of $<13$. Long-term data were collected on patients who achieved remission in the acute phase of treatment and who were available for followup. Data were collected at five time points: 6-12 months, 12 24 months, 24-36 months, 36-48 months, and 48 months or greater. $71.9 \%$ (23 out of 32 ) of patients maintained remission with an average of $\mathbf{1 4 . 3}$ months following the acute treatment phase with a $28.1 \%$ relapse rate. At 41.1 months, $90.0 \%$ (9 out of 10) of patients who were available for follow-up maintained remission. Three patients were available for follow-up data at over 5 years, with $66.7 \%$ (2 out of 3 ) patients maintaining remission at an average of 60.3 months. TMS was well tolerated and there were no patients who discontinued treatment due to adverse events.

CONCLUSION: In routine clinical practice, TMS shows significant improvements for treatment of major depression in a treatment-resistant population utilizing the Beck Depression Scale. In addition, long-term data further demonstrate the efficacy of TMS as a durable treatment option in a population that has failed multiple medication trials.

\section{9}

A retrospective analysis of transcranial magnetic stimulation right dorsolateral prefrontal cortex treatments for patients with generalized anxiety disorder

Kimberly Cress, $M D^{\prime}$; Sherry Grogan, APRN, PMHNP$B C^{\prime}$; Kerstin Brown, BA'; Beth Landry'; and Roxane Zotyka'

1 TMS Serenity Sugar Land, TX

ABSTRACT: Background: TMS therapy is FDA-approved for treatment of unipolar, non-psychotic major depressive disorder, but shows great promise for the treatment of generalized anxiety disorder. Our experience in treating patients with GAD with low-frequency, RDLPFC TMS therapy has shown significant improvement of anxiety symptoms.

METHODS: 17 patients with a diagnosis of generalized anxiety disorder were treated with low-frequency, rightsided TMS Therapy between 2011 and 2015. Patient diagnoses of GAD were based on DSM-IV and DSM-V criteria. Patients were treated only on the RDLPFC with a $1 \mathrm{~Hz}$ per second protocol for either 1,600 or 2,400 pulses. Patients were assessed using the Beck Anxiety Inventory scale. Scores were performed prior to and at the end of the acute treatment phase. Long-term results were reported on those patients that returned for assessment.

RESULTS: The study population included an average age of 38.7 years with $64.7 \%$ female. The mean TMS sessions were 34.2 with a range of 1,600 to 2,400 pulses. 10 patients $(58.8 \%)$ achieved remission. $64.7 \%$ of patients showed a response to TMS Therapy based on BAI response criteria of $50 \%$ improvement of symptoms. Long-term data were collected on patients who achieved remission in the acute phase of treatment and who were available for followup. Data were collected 6-12 months post-acute treatment on 14 patients who were available for follow-up assessment. $57.1 \%$ (8 out of 14 ) of patients maintained remission with an average of 11.9 months following the acute treatment phase with a $42.9 \%$ relapse rate. TMS therapy was well-tolerated and there were no patients who discontinued treatment due to adverse effects.

CONCLUSION: In routine clinical practice, TMS shows significant promise for treatment of generalized anxiety 
disorder using low-frequency, right-sided TMS therapy utilizing the Beck Anxiety Scale. Data on low-frequency RDLPFC TMS therapy are limited, but support RDLPFC treatment for those who suffer from GAD. Thus, further studies should be considered.

\section{0}

\section{Canonical loadings of PANSS subscales show differential placebo and aripiprazole drug responses in schizophrenia patients}

Srikanth Gottipati, $P h D^{\prime}$; Alejandro Ochoa, PhD', Shashank Rohatagi, PhD'; Andy Forbes, $\mathrm{PhD}^{\prime}$; Debbie Profit, PhD'; Raymond Sanchez, $M D^{\prime} ;$ Margaretta Nyilas, $M D^{\prime}$; and William Carson, $M D^{\prime}$

${ }^{1}$ Otsuka Pharmaceutical Development \& Commercialization, Inc., Princeton, NJ, USA

${ }^{2}$ Lewis-Sigler Institute for Integrative Genomics \& Center for Statistics and Machine Learning, Princeton University, Princeton, NJ, USA

ABSTRACT: Study Objectives: Lack of objective clinical endpoints is an essential problem in schizophrenia research. Disease state and treatment outcomes are measured using rating scales, e.g., Positive and Negative Syndrome Scale (PANSS), with their total scores as clinical endpoints. However, the PANSS total score cannot always differentiate between placebo and drug treatment, even for established antipsychotics. The goal of this study is to identify the individual items of PANSS that are most sensitive to differentiate between placebo and drug responses using canonical correlation analysis (CCA).

METHOD: Data from four placebo-controlled clinical trials (4-6 weeks long) in acutely relapsed or acute schizophrenic patients were analyzed. The data include longitudinal PANSS scores for 1368 subjects (397 subjects in the placebo cohort and 971 subjects in the drug cohort with dose ranging from $2 \mathrm{mg}$ /day to $30 \mathrm{mg}$ /day) who are predominantly male $(75 \%)$ and aged between 17 years and 76 years (median age 40 years). The trials were conducted at 149 medical centers in the US with multiple raters evaluating patients (rater information is unavailable to us). CCA was applied between all possible pairs of PANSS subscales grouped as Positive, Negative, and General Psychopathology scales. The canonical loadings were compared across cohorts, by dose, demographics, and by weeks from the start of the trial. Study identifiers: 31-97-201; 31-97-202; CN138-001; NCT00080327.

RESULTS: The results reveal significant patterns in canonical loadings of PANSS subscales (change from baseline) that differentiate placebo from drug response when observed in the first two canonical dimensions that show largest associations between pairs of subscales. Overall, the positive symptoms showed the most differential in canonical loadings between the placebo and drug cohorts. The results also show that fewer dimensions can reveal all the linear correlations between subscales in the drug cohorts as opposed to placebo. Furthermore, patterns in canonical loadings between PANSS subscales show clear structural shifts in the second week measurements since the start of the trial. By comparing and contrasting between subscales we have also eliminated much of rater biases and therefore minimized inter-rater variability.

CONCLUSIONS: By visualizing canonical loadings in several dimensions, CCA has revealed patterns in PANSS subscales that differentiate placebo from drug response and these patterns hold across trials. Future work will apply non-parametric methods (that detect nonlinearly correlated items as opposed to the CCA method, which is limited to linear correlations) in detecting signals of placebo and drug response.

FUNDING: This research was supported by Otsuka Pharmaceutical Development \& Commercialization, Inc.

\section{1}

\section{Significance of prolactin level changes associated with risperidone and aripiprazole use in youth}

\section{Sultana Jahan, $M D^{\prime}$ \\ ${ }^{1}$ Department of Psychiatry, University of Missouri- Columbia, Columbia, MO, USA}

ABSTRACT: Study Objectives: To evaluate the relationship between the use of common second generation antipsychotics (SGAs) on prolactin levels in a universitybased outpatient child and adolescent clinic over a period of six months.

METHODS: A clinical chart review was completed in youth treated with SGAs over a period of six months. The main SGAs used in the clinic were aripiprazole and risperidone. Data pertaining to demographics, duration of treatment, prolactin level, polypharmacy, and dosage were collected and analyzed. Descriptive data analyses, t-tests, and Pearson coefficients were used in the analysis. Literature was reviewed to understand the physiological role of prolactin in the body.

RESULTS: The aripiprazole group $(\mathrm{n}=24)$ had a mean prolactin level of 3.2 and median and mode of 2.8. The risperidone group $(\mathrm{n}=33)$ had a mean prolactin level of 27.9, a median of 25.7, and mode of 37.6. The prolactin level in the aripiprazole group was much lower than normal 
lab reference values, in contrast to the prolactin level in the risperidone group. The aripiprazole group was also associated with a lower risk of hyperprolactinemia $(0 \%)$ in contrast to the risperidone group ( $86 \%$ in males, $61 \%$ in females). An increased dose of aripiprazole was associated with an increased prolactin level (Pearson coefficient 0.53). Increased age was also associated with an increase in prolactin though no levels were above normal. No such relationship was present in the risperidone group.

CONCLUSIONS: As was expected in the literature for adults and youths, risperidone was associated with increased prolactin and aripiprazole with decreased prolactin. The potential of side effects of hyperprolactinemia with risperidone has been widely addressed (i.e., oligomenorrhea, amenorrhea, infertility, sexual dysfunction, osteoporosis, gynecomastia) but effects of hypoprolactinemia with aripiprazole on developing youths has been under studied and often gone unnoticed. Hypoprolactinemia can greatly influence the growth and development of young patients. Long-term low prolactin can lead to delayed puberty, subfertility, and infertility as well as immune depression. Further studies are necessary to enhance our knowledge of aripiprazole induced hypoprolactinemia and its effects on growth and development of children and adolescents and also essential to develop practice guidelines.

FUNDING: No funding.

\section{REFERENCES:}

Safer DJ, Calarge CA, Safer AM. Prolactin serum concentrations during aripirazole treatment in youth. Journal of Child and Adolescent Psychopharmacology. 2013; 23: 282-289.

De Hert M, Dobblaere M, Sheridan EM, Cohen D, Correll CU. Metaabolic and endocrine adverse effects of second-generation antipsychotics in children and adolescents: A systematic review of randomized placebo controlled trials and guidelines for clinical practice. European Psychiatry. 2011; 26: 144-158.

\section{2}

\section{Variation in prolactin levels with aripiprazole and} risperidone: what is the clinical significance?

\section{Sultana Jahan, $M D^{\prime}$}

${ }^{1}$ Department Of Psychiatry, University of MissouriColumbia, Columbia, MO, USA

OBJECTIVES: To evaluate the relationship between the use of common second generation antipsychotics (SGAs) on prolactin levels in a university-based outpatient child and adolescent clinic over a period of six months.

METHODS: A clinical chart review was completed in youth treated with SGAs over a period of six months after Internal Review Board approval. The main SGAs used in the clinic were aripiprazole and risperidone. Data pertaining to demographics, duration of treatment, prolactin level, polypharmacy, and dosage were collected and analyzed. Descriptive statistic, t-tests, and Pearson coefficients were used in the analysis. Literature was reviewed to understand the physiological role of prolactin in the body. Note: Prolactin levels cited are in $\mathrm{ng} / \mathrm{mL}$.

RESULTS: The aripiprazole group $(\mathrm{n}=24)$ had a mean prolactin level of 3.2 and median and mode of 2.8 . The risperidone group $(n=33)$ had a mean prolactin level of 27.9 , a median of 25.7 , and mode of 37.6. The prolactin level in the aripiprazole group was much lower than normal lab reference values, in contrast to the prolactin level in the risperidone group. The aripiprazole group was also associated with a lower risk of hyperprolactinemia $(0 \%)$ in contrast to the risperidone group $(86 \%$ in males, $61 \%$ in females).

Increased dose $(\mathrm{mg} / \mathrm{kg})$ of aripiprazole was associated with increasing prolactin level (Pearson coefficient, $\mathrm{r}=0.53$ ) and increased age was also associated with increase in prolactin level. Aripiprazole group was associated with a lower prolactin level with not a single subject of the group in the high lab reference range $(0 \% \mathrm{M}, 0 \% \mathrm{~F})$ and the risperidone group was associated with significant hyperprolactinemia (86\% M, 61\% F) using the lab reference range. The levels of prolactin were below normal in the aripiprazole treated group. Increased age was also associated with an increase in prolactin, though no levels were above normal. No such relationship was present in the risperidone group.

CONCLUSIONS: As was expected in the literature for adults and youths, risperidone was associated with increased prolactin and aripiprazole with decreased prolactin. The potential of side effects of hyperprolactinemia with risperidone has been widely addressed (i.e., oligomenorrhea, amenorrhea, infertility, sexual dysfunction, osteoporosis, gynecomastia) but effects of hypoprolactinemia with aripiprazole on developing youths has been under studied and often gone unnoticed. Hypoprolactinemia can greatly influence the growth and development of young patients. Long-term low prolactin can lead to delayed puberty, subfertility, and infertility as well as immune depression. Further studies are necessary to enhance our knowledge of aripiprazole associated hypoprolactinemia and its effects on developing brain and body of children and adolescents and are also essential to develop practice guidelines.

173

\section{Atypical presentation of spontaneous carotid cavernous fistula}

Suneetha Madhu, $M D^{\prime}$; Mubarak Dawaki, $\mathrm{MD}^{\prime}$; and Madhu Vijayappa, $M D^{\prime}$ 
${ }^{1}$ Spectrum Health, Michigan State University, Grand Rapids, MI, USA

INTRODUCTION: Carotid cavernous fistula (CCF) is an abnormal communication between the cavernous sinus and the carotid arterial system, which can occur spontaneously or due to trauma. Pulsating exopthalmus, bruit, and conjunctival chemosis are classical triad of $\mathrm{CCF}$. This is a retrospective case review. We present a case of atypical presentation of CCF.

RESULTS: 68 y/o male with history of unilateral nephrectomy presented with vertical diplopia due to isolated left 4th cranial nerve palsy associated with left sided headache and blurry vision in his left eye. On examination he had mild left eye ptosis. Head CT, CT angiogram of the head, and MRI of the brain were within normal limits. Cerebral angiogram showed a carotid cavernous fistula with arterial feeders from left ophthalmic artery draining into left cavernous sinus. Patient was noted to have significant weight loss in the last few months and patient had further workup to rule out any malignancy. Patient was scheduled for transvenous embolization of CCF after ophthalmological evaluation.

CONCLUSION: Spontaneous carotid cavernous sinus fistula can present as an isolated cranial nerve palsy or have other atypical presentation.

\section{4}

Mental health statistics and suicide prevention at St. Tammany Sheriff's Office

Sureshkumar Bhatt, MD,FCCP'

${ }^{1}$ St. Tammany Parish Sheriff's Office, Covington, LA, USA

INTRODUCTION: Mental health problems among inmates are always higher than mental health problems in the general population. There is very limited data showing proper healthcare for inmate populations. Mental health problems and correction environment are very challenging situations. High stress environment and loss of freedom can create multiple psychiatric problems or destabilize mental problems. Suicidal risk is very high among prisoners. Antisocial personality and malingering behavior are major obstacles among arrested inmates. Early detection of suicidal risk factors, proper monitoring of suicidal people, and timely treatment including counseling can reduce suicidal risk to a significant level.

METHOD: Mental health statistics were monitored in 2015 . Mental health screening was done at the time of arrest, and a detailed screening was done at the time of intake screening. All patients were classified according to Psychiatric Risk Index (PRI). Most serious patients (PRI 5) were identified and provided immediate treatment including being placed on suicidal watch. They all were monitored according to protocol. All patients were provided appropriate pharmacotherapy and psychotherapy during incarceration period and a safe discharge plan when released from the facility.

RESULT: All inmates were screened immediately at the time of arrival and detailed intake medical screening was done within 6 hours of booking. Average booking per month was 643 arrests. Total monthly mental health appointment was 624 (54\% of all scheduled sick call appointments). Psychiatrist appointment per month was 349 patients. $20 \%$ arrestees reported mental health illness, $7 \%$ reported previous suicidal attempt, and $1 \%$ reported active suicidal thoughts/intent at the time of arrival. 55-65\% of inmates were active drug users at the time of arrival, $27.9 \%$ of inmates were under the influence of alcohol, and $65-75 \%$ of inmates were using drugs and/or alcohol. $14.6 \%$ of inmates were on mental health medications. Average 50 inmates per month reported suicidal thoughts or intent and were placed on suicidal watch. Three suicidal attempts occurred in the jail in 2015, but none were successful. Three inmates were required restraint for protection from selfharm. Most inmates with mental illness are off their medications and have been lost to follow up with their psychiatrists. All patients were managed with standard treatment based on their psychiatric problem. Not a single mental health-related grievance was submitted during the year of 2015. Quality of Mental Health at STPSO exceeds the high standards of the national level.

175

\section{Addressing underutilization of clozapine in the hospital setting}

Julie Niedermier, $M D^{\prime}$

${ }^{1}$ Ohio State University, Columbus, OH, USA

STUDY OBJECTIVE(S): Utilization of clozapine varies across settings throughout the nation. This study examines the rate of utilization of clozapine in the two largest publically-funded psychiatric hospitals in the Central Ohio community.

METHOD: Data regarding the utilization rates were obtained from a retrospective review of patients hospitalized and receiving treatment with clozapine and compared to national statistics available about clozapine use. Demographic and diagnostic information was also analyzed. 
RESULTS: The findings support that clozapine is underutilized in this geographical area and that certain variables are predictive of patients most likely to be offered this treatment.

CONCLUSIONS: Given the potential benefits of clozapine use and known data about utilization rates in this area, the community has developed a collaborative care network in an effort to expand this treatment to appropriate patients.

FUNDING: no funding

\section{6}

Efficacy and safety of HLD200 in children with attention-deficit/hyperactivity disorder: results from a pivotal phase 3 trial

Valerie Arnold, $M D^{\prime}$; Steven Pliszka, $\mathrm{MD}^{2}$; Andrea Marraffino, PhD ${ }^{3}$; Norberto J. DeSousa, $M A^{4}$; Bev Incledon, $P h D^{4} ; F$. Randy Sallee, $M D, P h D^{4}$; Timothy Wilens, $\mathrm{MD}^{5}$; and Jeffrey Newcorn, $M D^{6}$

${ }^{1}$ University of Tennessee Health Science Center, Memphis, TN, USA

${ }^{2}$ The University of Texas Health Science Center at San Antonio, San Antonio, TX, USA

${ }^{3}$ Florida Clinical Research Center, LLC, Maitland, FL, USA

${ }^{4}$ Ironshore Pharmaceuticals \& Development, Inc., Camana Bay, Grand Cayman

${ }^{5}$ Harvard Medical School, Boston, MA, USA

${ }^{6}$ Icahn School of Medicine at Mount Sinai, New York, NY, USA

OBJECTIVE: Evening-dosed HLD200 delays the initial release of methylphenidate by approximately $8-10$ hours targeting the onset of clinically meaningful treatment effect upon awakening and lasting to the evening. The objective was to determine whether HLD200 improves control of ADHD symptoms, and at-home early morning and evening functioning versus placebo in children with attention-deficit/hyperactivity disorder (ADHD). Safety and tolerability were also assessed.

METHODS: This was a pivotal, randomized, double-blind, multicenter, placebo-controlled, parallel-group, phase 3 trial of HLD200 in children (6-12 years) with ADHD (NCT02520388). Subjects had current or prior response on methylphenidate. Following a screening period of $\leq 2$ weeks with a 3 - to 7-day washout, subjects were randomized (1:1) to HLD200 or placebo once-daily each evening for 3 weeks. After 1 week, the initial $40 \mathrm{mg}$ dose of HLD200 was titrated in $20 \mathrm{mg}$ weekly increments to $60 \mathrm{mg}$ and $80 \mathrm{mg}$, as tolerated, with a one-step downtitration permitted. The primary efficacy measure was the ADHD Rating Scale (ADHD-RS-IV) Total Score following 3 weeks of treatment. The key secondary efficacy measures were the Before School Functioning Questionnaire (BSFQ) and Parent Rating of Evening and Morning Behavior-Revised, Morning (PREMB-R AM) and Evening (PREMB-R PM) subscales following 3 weeks of treatment. Safety measures included treatmentemergent adverse events (TEAEs), with a focus on sleep and appetite.

RESULTS: Of 163 children enrolled across 22 sites, 161 were included in the intent-to-treat population. After 3 weeks of treatment, children on HLD200 achieved a significant improvement versus those on placebo in ADHD symptoms (least-squares [LS] mean ADHDRS-IV: 24.1 vs $31.2 ; \quad p=0.002)$, at-home early morning functioning (LS mean BSFQ: 18.7 vs 28.4; $\mathrm{p}<0.001$; LS mean PREMB-R AM: 2.1 vs 3.6; $\mathrm{p}<0.001$ ), and at-home evening functioning (LS mean PREMB-R PM: 9.4 vs $12.2 ; \mathrm{p}=0.002$ ). There were no serious TEAEs, and all reported TEAEs were consistent with the known effects of methylphenidate. All sleeprelated TEAEs were transient and mild or moderate in severity.

CONCLUSIONS: Following daily evening administration, HLD200 was well tolerated and demonstrated significant improvements in ADHD symptoms and both at-home early morning and evening functioning versus placebo in children with ADHD.

FUNDING: Funding for this study was provided by Ironshore Pharmaceuticals \& Development, Inc.

177

\section{Venlafaxine use in a woman with endometriosis:} case report

Vithyalakshmi Selvaraj, $\mathrm{MD}^{\prime}$; Marin Broucek, $M D^{\prime}$; Palanikumar Gunasekar, MBBS'; Siv Hour, $M D^{\prime}$; Caron Gray, $M D^{\prime}$; and James F. Smith Jr., $M D^{\prime}$

${ }^{1}$ Creighton University School of Medicine, Omaha, NE, USA

ABSTRACT: Venlafaxine is a serotonin-norepinephrine reuptake inhibitor (SNRI) antidepressant approved by the U.S. Food and Drug Administration for the treatment of major depression, generalized anxiety disorder, social anxiety disorder, and panic disorder. We here report a case report of a patient with endometriosis who experienced improvement in her menstrual bleeding, after initiating treatment with venlafaxine. To best of our knowledge, there are no published case reports of venlafaxine being used in patients with endometriosis. 
178

Neurological and psychiatric comorbidities assessment in the PRISM II study of extromethorphan/quinidine for treatment of pseudobulbar affect

William Sauve, $\mathrm{MD}^{\prime}$; David N. Alexander ${ }^{2}$; Andrew J. Cutler $^{3}$; Stephen D'Amico ${ }^{4}$; Flora M. Hammond ${ }^{5}$; Richard D. Zorowitz ${ }^{6}$; Andrea E. Formella ${ }^{7}$ and Joao Siffert ${ }^{7}$ *

${ }^{1}$ TMS NeuroHealth, Richard, VA, USA

${ }^{2}$ David Geffen School of Medicine at UCLA, Los Angeles, CA, USA

${ }^{3}$ Florida Clinical Research Center, LLC, Bradenton, FL, USA

${ }^{4}$ Cornerstone Medical Group, Franklin, TN, USA

${ }^{5}$ Indiana University School of Medicine, Indianapolis, IN, USA

${ }^{6}$ MedStar National Rehabilitation Network, Washington, DC, USA

${ }^{7}$ Avanir Pharmaceuticals Inc., Aliso Viejo, CA, USA

*Former employee of Avanir Pharmaceuticals Inc., Aliso Viejo, CA, USA

ABSTRACT: Objectives: Pseudobulbar affect (PBA) can occur secondary to certain neurological diseases or brain injury and is characterized by frequent, uncontrollable laughing/crying episodes. While PBA is distinct from mood disorders in which feelings of happiness or sadness can also lead to laughing or crying, persons with PBA may often have psychiatric comorbidities (e.g., depression or anxiety). A recently completed study (PRISM II) evaluated the effectiveness of dextromethorphan/quinidine $(\mathrm{DM} / \mathrm{Q})$ for the treatment of PBA in persons with dementia, stroke, and traumatic brain injury (TBI); we assessed the prevalence of comorbid neurological and psychiatric disorders among PRISM II patient cohorts.

METHODS: Patients with PBA secondary to either dementia, stroke, or TBI, and a Center for Neurologic StudyLability Scale (CNS-LS) score $\geq 13$ were enrolled and treated with DM/Q $20 / 10 \mathrm{mg}$ BID, open-label for 90 days. Persons with severe dementia, stroke within $\leq 3$ months, penetrating TBI, severe depressive disorders, or psychotic disorders were excluded. Concomitant medications for neuropsychiatric conditions were allowed provided doses were stable, and there were no contraindications to DM/Q use. Baseline assessments included PBA and depression ratings (CNS-LS, episode count, and PHQ-9) concomitant diseases and medications.

RESULTS: A total of 367 patients were enrolled $(\mathrm{n}=120$ TBI, $\mathrm{n}=113$ stroke, $\mathrm{n}=134$ dementia), $70.8 \%$ were receiving $\geq 1$ psychiatric medication at baseline, most commonly antidepressants $(48.5 \%)$, antipsychotics
(18.0\%), and sedatives/anxiolytics/hypnotics (33.8\%). A total of $57.5 \%$ reported a depression diagnosis at baseline, including $61.7 \%, 50.4 \%$, and $59.7 \%$ of the TBI, stllroke, and dementia populations, respectively; other reported CNS diagnoses included: anxiety disorders, $42.2 \%$ (50.0\% TBI, 36.3\% stroke, and $40.3 \%$ dementia, respectively); sleep disorders, $34.1 \%(40.8 \%, 29.2 \%$, and $32.1 \%)$; cognitive impairment, $28.9 \% \quad(25.8 \%$, $18.6 \%$, and $40.3 \%$ ); headache disorder, $20.4 \%(37.5 \%$, $15.9 \%$, and $9.0 \%)$; seizures, $14.7 \%(17.5 \%, 16.8 \%$, and $10.4 \%)$; post-traumatic stress disorder, $4.6 \%(13.3 \%$, $0.9 \%$, and $0.0 \%$; Parkinson's disease, $5.2 \%$ (0.8\%, $4.4 \%, 9.7 \%)$. The baseline mean PHQ-9 score was 13.5 (13.9, 13.4, and 13.2, respectively), suggesting moderate depression.

CONCLUSIONS: Persons enrolling in this study of DM/Q for treatment of PBA subsequent to TBI, stroke, or dementia often had other CNS comorbidities, most commonly, depression and anxiety disorders. These findings underscore the importance of considering both neurologic and psychiatric causes in the differential diagnosis of affective symptoms such as uncontrollable laughing and crying. The fact that over $70 \%$ of our study population was already being treated for psychiatric comorbidity suggests that specific PBA treatment may still be required.

\section{9}

\section{Lithium-induced neurotoxicity at therapeutic levels: illustrative cases and literature review}

Yara Moustafa, MD PhD'; Ivonne Torriente Crespo, $\mathrm{MD}^{\prime}$; Celine Corona, $\mathrm{MD}^{\prime}$; and John W. Stiller, $\mathrm{MD}^{\prime}$

${ }^{1}$ Saint Elizabeths Hospital DBH, Washington, DC, USA

BACKGROUND: Lithium has been successfully used as a mood stabilizer for treatment and prophylaxis of bipolar disorders. Lithium has a narrow therapeutic index that includes neurotoxic effects at levels above the "therapeutic range." However, under certain circumstances, significant neurotoxicity may occur at levels well within in the "therapeutic range." Although there are many case reports of delirium associated with therapeutic lithium levels that resolve with discontinuing or reducing the lithium dose, occasionally an overreliance on the "therapeutic level" results in no adjustment of the lithium dose, a prolonged diagnosis of "delirium of unknown cause," and rarely irreversible neurotoxicity.

CASE PRESENTATION: We present two cases of lithium neurotoxicity within therapeutic lithium levels.

CASE 1: A 76-year-old man with bipolar I disorder on lithium $300 \mathrm{mg}$ BID was admitted to a psychiatric 
hospital with a diagnosis of mixed manic-depressive disorder, a recognized diagnosis with DSM-5. On admission, he was confused and had difficulty with balance. Lithium level was 0.71 , which is within the "therapeutic range" [0.6-1.2], and he was continued on the $300 \mathrm{mg}$ BID. A complete work-up for his delirium (including CBC, CMP, UA, arterial blood gases, head CT, brain MRI, EEG, LP, and CXR) revealed no clear cause, and a neurological consultation agreed with the diagnosis of delirium, and suggested lithium neurotoxicity despite therapeutic levels. The patient's delirium improved and ultimately resolved after discontinuing the lithium.

CASE 2: A 54-year-old man with schizophrenia on clozapine $300 \mathrm{mg}$ BID and lithium $300 \mathrm{mg}$ BID presented with recurrent falls associated with postural instability. Lithium level was 0.6. His motor difficulties resolved after lithium was discontinued.

DISCUSSION: We review the literature on lithium-induced neurotoxicity at therapeutic levels including other relevant case reports and possible explanations for the neurotoxic effects, including the possibility of interfering with signal transduction through G-protein-coupled receptor (GPCR) pathways and/or N-methyl-D-aspartate receptor (NMDAR)/nitric oxide (NO) signaling pathway involvement. The syndrome of irreversible lithiumeffectuated neurotoxicity (SILENT) will also be discussed.

CONCLUSION: Our cases and others reported in the literature highlight the importance of maintaining a high index of suspicion for lithium neurotoxicity in patients with unexplained neurological dysfunction on lithium despite therapeutic serum levels. Because lithium neurotoxicity may occur with "therapeutic" serum levels, and may occasionally be irreversible, lithium should be discontinued until it is clear that it is not contributing to the neurological dysfunction.

\section{0}

\section{Predictors of re-hospitalization in patients with serious mental illnesses}

Yijun Shao, PhD'; Srikanth Gottipati, PhD ${ }^{2}$; Pravin Jadhav, PhD ${ }^{2}$; Akshay Vashist, PhD ${ }^{2}$; Shashank Rohatagi, PhD ${ }^{2}$; Andy Forbes, $P h D^{2}$; Debbie Profit, PhD ${ }^{2}$; Raymond Sanchez, $\mathrm{MD}^{2}$; Margaretta Nyilas, $\mathrm{MD}^{2}$; William Carson, $\mathrm{MD}^{2}$; Yan Cheng, $\mathrm{MS}^{I}$; and Qing Zeng-Treitler, $P h D^{\prime}$

\footnotetext{
${ }^{1}$ Medical Informatics Center, School of Medicine and Health Sciences, George Washington University, and VA Medical Center, Washington, DC, USA

${ }^{2}$ Otsuka Pharmaceutical Development \& Commercialization, Inc., Princeton, NJ, USA
}

ABSTRACT: Study Objectives: The re-hospitalization rate is an important clinical quality measure, because hospitalization is a poor outcome and costly. Prior studies have explored factors associated with risk of hospitalization in patients with mental illnesses but knowledge gaps still exist. Studies that examined clinical factors were often performed on small samples. Cognitive behavior therapies and other supportive therapies have not been analyzed in depth. We intend to address these issues using Electronic Health Records (EHR) data.

METHODS: Using the national clinical data repository developed from the Veteran Healthcare Administration's EHR, we identified a cohort of 1.2 million adults with serious mental illnesses (schizophrenia (SZ), schizoaffective disorder $(\mathrm{SAD})$, bipolar disorder $(\mathrm{BP})$, or major depressive disorder (MDD)) from 2001 to present. To have sufficient pre-diagnosis/hospitalization data and a long enough follow up period to analyze the predictors, the cohort was further restricted to patients with the 1st diagnosis of SZ, SAD, BP, and MDD to after 2003 and $\geq 5$ years of follow-up data. A multivariable logistic regression analysis was performed. Collinearity was checked using a correlation co-efficient with a threshold of 0.75 . The primary outcome variable was $3-y r$ rehospitalization. The independent variables $(n=97)$ were demographics, primary diagnosis of the 1st hospitalization, and diagnoses, procedures, visit types within 1 year prior to the 1st hospitalization.

RESULTS: The final cohort contained 79,734 cases $(>=1$ hospitalization within 3 years of the 1 st hospitalization) and 60,096 controls. The cases were $90.1 \%$ male and $70.2 \%$ white and the controls were $87.5 \%$ male and $79.5 \%$ white. Certain social and behavior factors were found to be predictors including lack of housing ( $\mathrm{OR}=$ 1.28, $\mathrm{p}<0.01)$ and alcohol $(\mathrm{OR}=1.03, \mathrm{p}<0.01)$, cocaine $(\mathrm{OR}=1.40, \mathrm{p}<0.01)$, opioid $(\mathrm{OR}=1.16$, $\mathrm{p}<0.01)$, and other drug abuse $(\mathrm{OR}=1.15, \mathrm{p}<0.01)$. Interestingly, unemployment was not a predictor and cannabis use was negatively associated with rehospitalization $(\mathrm{OR}=0.94, \mathrm{p}<0.01)$. Counseling was also associated with no re-hospitalization $(\mathrm{OR}=0.94$, $\mathrm{p}<0.01)$.

CONCLUSION: We have identified a large cohort of serious mental illness patients with longitudinal data. The large sample size allowed a nuanced examination of the predictors of re-hospitalization. Our first analysis focused on the social/behavior predictors and counseling. The results were mostly consistent with prior literature with several unexpected findings. A limitation of this study is that it only used structured data; in future work we plan to further refine the analysis by adding 
information extracted from free notes. In addition, we plan to explore treatment effects on re-hospitalization.

FUNDING: This research was supported by Otsuka Pharmaceutical Development \& Commercialization, Inc.

\section{1}

Eicosapentaenoic acid-, phosphatidylserine-, and docohexaenoic acid (Vayarin)-induced spitting in Tourette's syndrome

\author{
Zeenat Burero'; and Alan R. Hirsch, MD, FACP \\ ${ }^{1}$ Final year medical student, Ziauddin University, \\ Karachi, Pakistan \\ ${ }^{2}$ Smell and Taste Treatment and Research Foundation, \\ Chicago, IL, USA
}

ABSTRACT: Introduction: Vayarin, which is $21.5 \mathrm{mg}$ eicosapentaenoic acid, $75 \mathrm{mg}$ phosphatidylserine, and $8.5 \mathrm{mg}$ docosahexaenoic acid has not been reported to exacerbate Tourette's Syndrome (TS) or precipitate expectorations. Such a case is presented.

METHOD: Case study: A 14-year-old, right handed, male presented with a six year history of poor attention, impaired concentration, decreased focus, and day dreams. At age of eight, he was treated with amphetamine and dextroamphetamine for one day, which caused a tic to develop, forcing the patient to stop the medication, whereupon the tic stopped. He continued to have attention difficulties and began mumbling tetragrams under his breath. He affirmed obsessions about movies occurring everyday, almost all day. His parents complained that he was constantly chewing nonfood objects, for instance clothing, pencil, and screws. The patient was started on Vayarin BID, and within a few hours, he began uncontrolled spitting onto the floor. This persisted and was of such great magnitude that the floor was literally covered by a puddle of saliva. Such compulsive expectoration has been unrelenting despite discontinuation of Vayarin.

RESULTS: Abnormalities in physical examination: General: Occasional tic of right shoulder and face: Frequently bombilates poorly articulated tetragrams. Neurological Examination: Gait Examination: adventitious movements with dystonic posturing of both upper extremities on heel walking. Neuropsychiatric test: Clock drawing test: 4 (normal). Animal Fluency Test: 24 (normal). Go-No-Go test: 6/6 (normal). Other: ceruplasmin, creatinine phosphokinase, complete blood count, liver function tests: normal.

DISCUSSION: Vayarin possibly enhances dopamine production which functions in the striatum to facilitate expectorant tic variant. The taste of Vayarin may be bitter or hedonically negative-so bitter as to induce disgust and spitting. Vayarin induced reflux may cause nausea and the bitter taste of gastric contents, serving as a nidus for spitting. The expectoration could have become a habit which transformed into a compulsion. This may not be due to a tic but rather due to an unconscious psychological response, a psychodynamically primitive way of rejecting the treatment option. Rather than being distracted as a result of attention deficit/hyperactivity disorder (ADHD) the Vayarin may improve his ADHD and enhance concentration, thus allowing him to focus on any underlying obsession and compulsion (spitting). Ironically, through its intended mechanism of action, Vayarin may worsen any obsessive compulsive symptoms of TS. In those suffering from TS, movement, or compulsive disorders, change in tics, coprophenomena, and compulsions should be assessed after use of Vayarin.

182

\section{Genetic risk factors associated with commonly prescribed psychotropic medications}

Zia Choudhry, MD, PhD'; Lorna Khensouvann, $M S^{\prime}$; Kathryn Gardner, $M S^{\prime}$; Michelle Martinez, $B S^{\prime}$; and Jay Lombard, $D O^{\prime}$

${ }^{1}$ Genominc, Inc., King of Prussia, PA, USA

STUDY OBJECTIVES: Approximately 10 million American adults suffer from a serious mental illness ever year, including schizophrenia, bipolar disorder, or major depressive disorder. Psychiatrists have treated patients using the traditional trial-and-error approach, but psychotropic medications are well-known for associated adverse events that are prevalent and problematic. Many of these medications cause intolerability that may lead to low medication adherence and treatment resistance. Several studies have demonstrated that adverse events are related to clinically actionable genetic variations. Therefore, pharmacogenetics testing proves to be a valuable tool in identifying these variations, considering clinicians can use a patient's unique genetic information to develop a personalized therapeutic plan, rather than applying a one-size-fits-all model. A personalized approach allows clinicians to avoid side effects, deduce correct dosing, and add certain medications to the regimen, which may yield better therapeutic outcomes for a patient. This study aims to investigate the potential risk for gene-drug interactions for the top 25 most commonly prescribed psychotropic drugs.

METHODS: Genetic variation data were obtained from a database of 25,754 psychiatric patients who received the Genecept AssayT (Genomind, Inc., King of Prussia, PA). 
The frequencies of clinically actionable variations in the genes SLC6A4, CACNA1C, ANK3, 5HT2C, DRD2, COMT, MTHFR, CYPs 2D6, 2C19, and 3A4/5 were calculated for this patient population. The frequencies were further evaluated in relation to the top 25 most commonly prescribed psychotropic medications in 2013 that were reported by the IMS Health. These psychotropic medications include antidepressants, antipsychotics, anxiolytics, and dopaminergic stimulants.

RESULTS: Frequency data for genetic variations in this patient population match up with the Caucasian population frequencies reported in dbSNP. Further, data show that the potential for a gene-drug interaction with these commonly prescribed psychotropic medications is highly probable. More specifically, out of 25,754 individuals in this data set, more than $99.7 \%$ had at least one clinically actionable variation in the 10 genes evaluated by the Genecept Assay.

CONCLUSION: Data from these patients reflect a high risk for gene-drug interactions with commonly prescribed psychotropic medication. These data also add to the growing evidence that pharmacogenetics testing is a useful tool in treating psychiatric patients. Further, providing clinicians with individual patient's genetic results can improve treatment outcomes by reducing the risk of adverse events, nonresponse, and treatment resistance, while increasing medication adherence. If pharmacogenetic testing is implemented in clinical practice and becomes a gold standard, this may ultimately decrease healthcare expenses and increase the number of psychiatric patients who experience improved response and remission.

FUNDING: no funding

\section{3}

\section{Algorithm for adult attention deficit hyperactivity} disorder management from the psychopharmacology algorithm project at the Harvard South Shore program

\footnotetext{
Bushra Awidi, $M D^{\prime}$; and David Osser, $M D^{1,2}$

${ }^{1}$ VA Boston Healthcare System/Harvard Medical School

${ }^{2}$ Algorithm for Adult Attention Deficit Hyperactivity Disorder Management from the Psychopharmacology Algorithm Project at the Harvard South Shore Brockton, MA
}

ABSTRACT: Objective: ADHD is a neurodevelopmental disorder with a worldwide rate of approximately $3-10 \%$ in school-age children. It often continues to show manifestations in adults, with up to $4 \%$ diagnosed adults worldwide. These patients suffer from a multitude of functional impairments with overall negative impacts on their quality of life.

METHOD: A medication algorithm for adult $\mathrm{ADHD}$ was created using systematic literature search to identify relevant studies and key findings. We prioritized treatment considerations based on the following: 1) effectiveness and efficacy, 2) co-morbidity with other psychiatric or medical conditions, and 3) safety and longterm tolerability.

RESULTS: After an accurate diagnosis of adult ADHD and after accounting for any comorbidity that may affect the algorithm, we propose initiating treatment with a low dose $(5 \mathrm{mg})$ of methylphenidate (MPH) or amphetamine once daily and titrating the dose every 3 days until effectiveness occurs or until side effects develop, with the usual efficacious dose being $1-1.3 \mathrm{mg} / \mathrm{kg}$ for MPH and $0.6-0.9 \mathrm{mg} / \mathrm{kg}$ for amphetamines. In adult ADHD and co-morbid substance use, we recommend deferring ADHD pharmacotherapy until a period of sobriety has been established, after which the first line medication would be atomoxetine at a target dose of $1.2 \mathrm{mg} / \mathrm{kg}$. In patients who develop mania or psychotic symptoms while on stimulants, we recommend discontinuing stimulants and reconsidering your diagnosis. In patients with established bipolar disorder, we recommend stabilizing mood with mood stabilizers followed by a slow and careful addition of low dose stimulants along with close monitoring of symptoms as this patient population remains at a higher risk for developing mania.

CONCLUSION: This algorithm is supported by the available but limited latest evidence and was created in response to the growing need for a treatment guide to clinicians when choosing medications for adult $\mathrm{ADHD}$.

\section{4}

\section{The use and value of the 7-Item Binge Eating Disorder Screener in clinical practice}

Barry K. Herman, MD, $M M M^{\prime}$; Linda S. Deal, $M S c^{2}$, Dana B. DiBenedetti, PhD ${ }^{3}$; Lauren Nelson, $P h D^{3}$ Sheri E. Fehnel, PhD ${ }^{3}$; and T. Michelle Brown, PhD ${ }^{3}$

${ }^{1}$ Lexington, MA

${ }^{2}$ Philadelphia, PA

${ }^{3}$ RTI Health Durham, NC

ABSTRACT: Study Objectives: Evaluate physician knowledge and attitudes about binge eating disorder (BED) and describe the value and ease of use of the 7-Item Binge Eating Disorder Screener (BEDS-7) in clinical practice. 
METHOD: Two internet surveys (wave 1 [Apr 15-May 6, 2015]; wave 2 [Aug 19-25, 2015]) were administered to general practitioners (GPs) and psychiatrists. Wave 1 invitees were randomly selected from a panel of US-based physicians spending $\geq 50 \%$ of their time in direct patient care and reporting "no" to "some to average" experience with eating disorder patients; those completing wave 1 qualified for wave 2 . The surveys assessed BED knowledge, beliefs, and attitudes and the value and ease of use of the BEDS-7. Continuous variables were analyzed with $t$ tests and categorical variables with chi-square or Fisher exact tests.

RESULTS: 245 physicians (122 GPs; 123 psychiatrists) completed both waves. Composite BED knowledge (percent correct) increased significantly from wave 1 to wave 2 in GPs $(55.2 \%$ to $60.0 \%, \mathrm{P}<0.001)$ and psychiatrists $(73.0 \%$ to $76.4 \%, \mathrm{P}<0.05)$; knowledge of GPs was lower than psychiatrists (both waves, $\mathrm{P}<0.001)$. Mean ąSD composite belief scores about the importance of being knowledgeable about BED ( maximum $=6$; higher scores $=$ greater belief) were high in GPs and psychiatrists in both waves (range, 5.14 ą1.3 to 5.40 ą 0.9$)$. Mean ą SD composite comfort scores for diagnosing and treating BED (maximum $=6$; higher scores $=$ more comfort) were significantly lower in GPs than psychiatrists in both waves (wave 1: 3.34 a 1.6 vs 4.47 ą $1.3, \mathrm{P}<0.001$; wave 2 : 3.06 ą 1.7 vs 4.37 ą 1.4 , $\mathrm{P}<0.001)$. The BEDS-7 was used in clinical practice by $32.0 \%$ of GPs and $26.8 \%$ of psychiatrists. During wave 1, larger percentages of BEDS-7 users versus nonusers anticipated that the BEDS-7 would be "very valuable" (GPs: $82.1 \%$ vs $37.3 \%, \mathrm{P}<0.001$; psychiatrists, $54.5 \%$ vs $34.4 \%$ ); all BEDS-7 users reported the BEDS-7 to be "very" or "somewhat" valuable during wave 2 . During wave 1, larger percentages of BEDS-7 users versus nonusers anticipated that the BEDS-7 would be "very easy" to use (GPs: $53.8 \%$ vs $38.6 \%$; psychiatrists, $60.6 \%$ vs $45.6 \%$ ); nearly all BEDS-7 users (GPs: 97.4\%; psychiatrists: $100 \%$ ) reported the BEDS-7 to be "very" or "reasonably" easy to use during wave 2. A majority of BEDS-7 users (GPs and psychiatrists) reported that important uses of the BEDS-7 included assisting clinicians in identifying patients with BED $(71.8 \%$ and $87.9 \%)$ and encouraging/ initiating doctor-patient discussions about BED $(71.8 \%$ and $66.7 \%$ ).

CONCLUSIONS: Knowledge of and comfort with diagnosing and treating BED were higher in psychiatrists than GPs, but both groups acknowledged the importance of being knowledgeable about BED. Among those who used the BEDS-7, it was reported to be a highly valued, easy-to-use screener for BED.
185

A double-blind, placebo-controlled, randomizedwithdrawal study of lisdexamfetamine dimesylate in adults with moderate to severe binge eating disorder

James Hudson, $\mathrm{MD}, \mathrm{Sc} \mathrm{D}^{\prime}$; Susan McElroy, $\mathrm{MD}^{2}$, M. Celeste Ferreira-Cornwell, PhD ${ }^{3}$; Jana Radewonuk, $\mathrm{MSc}^{3}$; Maria Gasior, MD, PhD ${ }^{4}$; and Barry K. Herman, $M D, M M M^{5}$

\footnotetext{
${ }^{1}$ McLean Hospital/Harvard Medical Middleborough, MA

${ }^{2}$ Lindner Center of Mason, $\mathrm{OH}$

${ }^{3}$ GlaxoSmith Collegeville, PA

${ }^{4}$ Philadelphia PA

${ }^{5}$ Lexington, MA
}

ABSTRACT: Study Objective: The objective of this study was to assess the maintenance of efficacy of lisdexamfetamine dimesylate (LDX) in adults with protocol-defined moderate-to-severe binge eating disorder (BED).

METHODS: Adults (18-55 years) meeting Diagnostic and Statistical Manual of Mental Disorders, Fourth EditionText Revision criteria for BED and having protocoldefined moderate-to-severe BED ( $\geq 3$ binge eating days/ week for 14 days before open-label baseline and Clinical Global Impressions - Severity (CGI-S) scores $\geq 4$ [at least moderately ill] at screening and open-label baseline) were eligible. Following a 12-week open-label phase (dose optimization: 4 weeks [50 or $70 \mathrm{mg}$ LDX]; dose maintenance: 8 weeks), protocol-defined LDX responders (participants having $\leq 1$ binge eating day/week for 4 consecutive weeks and CGI-S scores $\leq 2$ [borderline ill or less] at week 12) were randomized to receive placebo or to continue dose-optimized LDX treatment during a 26-week, double-blind, randomized-withdrawal phase. Time to relapse $(\geq 2$ binge eating days/week for 2 consecutive weeks and $\geq 2$ point CGI-S score increases from randomized-withdrawal baseline) was assessed in the full analysis set (FAS) using a stratified log-rank test stratifying for 4-week cessation. Safety and tolerability assessments included treatment-emergent adverse events (TEAEs).

RESULTS: Of 418 enrolled participants, 275 LDX responders (placebo, $\mathrm{n}=138$; LDX, $\mathrm{n}=137$ ) entered the randomized-withdrawal phase. LDX demonstrated superiority over placebo $(\mathrm{P}<0.001)$ for time to relapse. The observed proportions of participants meeting relapse criteria were $3.7 \%$ (5/136) for LDX and $32.1 \%(42 / 131)$ for placebo. TEAEs reported by $>5 \%$ of participants during open-label treatment were dry mouth (33.8\%), headache $(16.1 \%)$, insomnia (11.2\%), decreased appetite $(9.2 \%)$, nausea $(8.5 \%)$, anxiety $(7.1 \%)$, constipation $(6.8 \%)$, hyperhidrosis $(5.6 \%)$, feeling jittery $(5.1 \%)$, and diarrhea $(5.1 \%)$. 
CONCLUSIONS: Following initial LDX response, risk of relapse to binge eating over 6 months was lower with continued LDX than with placebo. The safety and tolerability of LDX was consistent with its known profile.

FUNDING: Shire Development LLC.

\section{0}

\section{rTMS and ECT, are they combinable?}

Samir Ibrahim, M.B.CH.B., Master Psychiatry, F.R.A.N.Z.C.P. '; and Paul FitzgeraldProf, MARPcr ${ }^{2}$

${ }^{1}$ Sydenham Specialists Clinic, Northpark Private

Hospital, Wyndham Private Victoria, Australia

${ }^{2}$ Monash University, Victoria, Australia

ABSTRACT: Background: Depression, although known since antiquity, remains on top of the research list of therapeutic interventions. Difficult to treat depression continues to pose challenging management in at least $1 / 3$ of cases (STAR D), and almost $21 \%$ in Primary Care (McIntyre 2014).

Management of depression, so far, has less than satisfactory results, a fact frustrating the therapists and the consumers, equally (Souery et al., 2006).

Many studies and meta-analysis have compared efficacy of rTMS and ECT, using several versions of each of the neuro-stimulation therapeutic tools (Berlim et al., 2013).

Dannon (2002), Grunhaus (2000), and Janicak (2002) published comparative studies, with an overall claim that ECT and rTMS are comparable in some aspects of improvement.

Pridmore (2000) substituted rTMS to ECT. He recruited 22 patients, who were randomized to receive either ECT over two weeks, or one ECT per week, followed by TMS for the rest of the week. He published results of comparative improvement, while the rTMS group had less cognitive side effects.

OBJECTIVES: This is a series of case reporting of nine patients suffering MDD, chronic and treatment resistant, who required both treatments (rTMS and ECT), alternating, in treating the same episode. All patients were on multiple pharmaco-therapeutic agents, and attended psychotherapy.

The hypothesis is that the combination can offer a good therapeutic tool for difficult to treat depression patients, with reducing the possible side effects incurred by ECT.

METHODS: Anterograde naturalistic study of the nine patients. No inclusion or exclusion criteria were considered.

FINDINGS: Patients have benefited from the combination therapy. This was measured by CGI and HAMD scores, which were measured before, during, and after the treatments combined.
CONCLUSION: This case report needs to be considered in designing further well controlled studies, to standardize the findings, and to negate the alleged hypothesis.

\section{Applying acupuncture to the treatment of addiction}

Jessica S. Bayner, $M D^{\prime}$; Ammar Ahmad, MD'; Ananya Sreepathi, $\mathrm{MD}^{\prime}$; and Hossain Asghar, $M D^{\prime}$

${ }^{1}$ Bergen Regional Medical Center, Paramus, NJ, USA

ABSTRACT: Addiction has been primarily addressed by detoxification protocols that are intended to mitigate the symptoms and discomfort associated with withdrawal. Yet targeting the behavioral activity associated with addiction has been far more elusive. The United States National Acupuncture Detoxification Association (NADA) protocol has been implemented as a method to relieve withdrawal symptoms, as well as the addictive behaviors related to symptoms of craving. Symptoms of withdrawal are thought to be associated with diminished dopamine levels in the brain after reduction or termination of the substance. Acupuncture is mechanistically thought to enhance dopamine levels in the brain by activation of opioid receptors, which modulate the mesolimbic dopamine system, the primary source that directs the brain's pleasure centers. This mechanism is hypothetically thought to be influenced by the release of endogenous opioids such as endorphin, enkephalin, dynorphin, and endomorphin, thereby modulating GABAergic interneurons in the ventral tegmental area and presynaptic?-opioid receptors in the nucleus accumbens. As many substances-including cocaine, amphetamines, opioids, alcohol, nicotine-share a common end pathway, the NADA protocol for acupuncture shows potential in regards to treating both the symptoms of withdrawal and the behavior comorbidity that can lead to relapse. Acupuncture (including electroacupuncture) as an adjuvant therapy along more traditional detoxification protocols has also been considered as a promising additional modality. Its reported efficacy in pregnant women in need of detoxification is also of interest, for those who prefer to refrain from using pharmacotherapy and avoid exposure to medication in utero. With respect to acupuncture and its ability to treat addiction as a whole, studies thus far have been mixed, as methodological quality has more often been poor and trials have been limited in regards to randomized controls. This literature review will address how acupuncture may be discussed as a topic for future exploration and studies in regards to the efficacy of the NADA protocol. Moreover, this review is intended to define the mechanisms that implicate acupuncture as a potential treatment modality, and the effects on behavioral activity associated with addiction. 